\title{
HARM VOSKUIL
}

\section{Non archimedean Hopf surfaces}

Séminaire de Théorie des Nombres de Bordeaux, tome 3, $\mathrm{n}^{\circ} 2$ (1991), p. $405-466$

<http://www.numdam.org/item?id=JTNB_1991_3_2_405_0>

(C) Université Bordeaux 1, 1991, tous droits réservés.

L'accès aux archives de la revue "Séminaire de Théorie des Nombres de Bordeaux » (http://jtnb.cedram.org/) implique l'accord avec les conditions générales d'utilisation (http://www.numdam.org/conditions). Toute utilisation commerciale ou impression systématique est constitutive d'une infraction pénale. Toute copie ou impression de ce fichier doit contenir la présente mention de copyright.

\section{Numdam}

Article numérisé dans le cadre du programme

Numérisation de documents anciens mathématiques

http://www.numdam.org/ 
Séminaire de Théorie des Nombres, Bordeaux 3 (1991), 405-466

\title{
Non archimedean Hopf surfaces.
}

\author{
par HARM VOSKUIL
}

\section{Introduction}

We study the non-archimedean Hopf surfaces. A Hopf surface is a surface defined over a complete field $K$, which has $K^{2}-\{(0,0)\}$ as its universal covering. So it can be described as $K^{2}-\{(0,0)\} / \Gamma$, where $\Gamma$ is a discrete group acting discontinuously on $K^{2}-\{(0,0)\}$.

The complex Hopf surfaces are very well-known. They have been studied in detail by Kodaira (See [Ko.1] and [Ko.2]).

The $p$-adic Hopf surfaces are less known, although they are treated as examples in some articles (See [GG], [Mus.1], [Mus.2] and [U]).All those articles mention only the diagonal Hopf surfaces $K^{2}-\{(0,0)\} / \Gamma$ with $\Gamma$ generated by a single element $\gamma$ such that $\gamma\left(z_{1}, z_{2}\right)=\left(\alpha z_{1}, \beta z_{2}\right)$ with $|\alpha|,|\beta|<1$. The most detailed study is given by Mustafin (See [Mus.1] and [Mus.2]). So there will be some overlap with his work.

This article is divided into three parts. In the first paragraph we will describe the group $\Gamma$. We will prove that $\Gamma \simeq \mathbb{Z} / l \mathbb{Z} \times \mathbb{Z}$ for some $l \in \mathbb{Z}_{>0}$. So these results are the same as in the complex case.

In the second paragraph we will give some pure affinoid coverings of a Hopf surface $X$, such that the reduction consists of non-singular components. Here we will use the theory of toroidal embeddings (see [KKMS], $[0,1]$ and $[0.2])$.

In the third paragraph we will determine the cohomology of the line bundles on a Hopf surface. We wil show that there is a Serre duality for the line bundles. This is also stated in [U] when $\operatorname{char}(K)=0$.

This paper is part of the author's doctoral dissertation at the university of Groningen in 1990. The author would like to express his gratitude to his thesis advisor Marius van der Put.

Manuscrit reçu le 7 mai 1991. 


\section{The structure of the group $\Gamma$}

We will first recall some basic definitions.

Definitions. Let $K$ be a complete non-archimedean valued field.

An affinoid algebra $A$ over $K$ is a $K$-algebra which is a finite extension of $\left.K<z_{1}, \ldots, z_{n}\right\rangle$ for some $n$.

An affinoid space $S p(A)$ is the set of all maximal ideals of the affinoid algebra $A$.

On $A$ we define a (semi-) norm : the spectral (semi-) norm $\|f\|=\sup _{x \in S p(A)}|f(x)|$. The spectral semi-norm is a norm if there are no nilpotent elements $\neq 0$ in $A$.

Example : The set $Y=\left\{\left(z_{1}, z_{2}\right) \in K^{2}|| z_{1}|\leq 1,| z_{2} \mid \leq 1\right\}$ is an affinoid space. The affinoid algebra belonging to $Y$ is $K<z_{1}, z_{2}>$.

Definitions. A surface $Y$ is called separated if $Y$ has an admissible affinoid covering $\left\{Y_{i} \mid i \in I\right\}$ such that if $Y_{i} \cap Y_{j} \neq \varnothing$ then $Y_{i} \cap Y_{j}$ is affinoid and the canonical homomorphism $\mathcal{O}\left(Y_{i}\right) \widehat{\otimes} \mathcal{O}\left(Y_{j}\right) \rightarrow \mathcal{O}\left(Y_{i} \cap Y_{j}\right)$ is surjective.

We write $U \Subset S p(A)$ and say $U$ is relatively compact in $S p(A)$ if there exists an affinoid generating system $\left\{f_{1}, \ldots, f_{r}\right\}$ of $A$ over $K$ such that :

$$
U \subset\left\{x \in S p(A)|| f_{1}(x)|<1, \ldots,| f_{r}(x) \mid<1\right\} .
$$

A surface $Y$ is called proper over $K$ if $Y$ is separated and has two finite affinoid coverings $\left\{X_{i}^{(1)} \mid i=1 . . n\right\}$ and $\left\{X_{i}^{(2)} \mid i=1 . . n\right\}$ such that $X_{i}^{(1)} \Subset X_{i}^{(2)}$ for all $i=1 . . n$.

A Hopf surface is a proper rigid analytic surface that has $K^{2}-\{(0,0)\}$ as its universal analytic covering.

Remark. In [U] a surface that we call proper is called compact.

In order to show that our definitions of a Hopf surface is meaningful we have to show that $K^{2}-\{(0,0)\}$ is simply connected. We will do this in the following lemma.

Definition. A connected analytic space $X$ is called simply connected if the only connected analytic covering of $X$ is equal to $i d: X \rightarrow X$.

Lemma 1.1. The analytic space $K^{2}-\{(0,0)\}$ is simply connected. 
Proof. Let us write $U=K^{2}-\{(0,0)\}=U_{1} \cup U_{2}$, where $U_{1}=K^{*} \times K$ and $U_{2}=K \times K^{*}$. Since $K$ and $K^{*}$ are simply connected, the same is true of $K \times K^{*}$ and $K^{*} \times K^{*}$ (This is theorem 1 in [vdP]). Now $U_{1} \cup U_{2}$ is also simply connected, since $U_{1}, U_{2}$ and $U_{1} \cap U_{2}$ are simply connected. Indeed let $S$ be a locally constant sheaf on $U_{1}$, then $S_{\left.\right|_{i}}$ is constant since $U_{i}$ is simply connected (See [vdP]). This shows that $S$ is constant on $U$, since $\left.S\left(U_{1}\right)\right|_{U_{1} \cap U_{2}}=\left.S\left(U_{2}\right)\right|_{U_{1} \cap U_{2}}$.

Definition. A group $\Gamma$ acts discontinuously on an analytic space $X$ if for every affinoid subspace $A \subset X$ the set $\{\gamma \in \Gamma \mid A \cap \gamma(A) \neq \varnothing\}$ is finite.

Lemma 1.2. A Hopf surface is a proper rigid analytic surface of the form $K^{2}-\{(0,0)\} / \Gamma$. Here $\Gamma$ is a group of automorphisms of $K^{2}-\{(0,0)\}$ that acts discontinuously and without fixed points.

Proof. The universal covering space of a Hopf surface $X$ is $U=K^{2}-\{(0,0)\}$. Let $\pi$ be the analytic map $\pi: U \rightarrow X$. Let $\Gamma$ be the group of covering transformations of $U$, so $\Gamma=\{\gamma: U \rightarrow U \mid \pi \circ \gamma=\pi\}$.

We have to show that $U / \Gamma \simeq X$. Clearly $\Gamma$ is discrete and $U / \Gamma \rightarrow X$ is a covering of $X$. So we only have to prove that $U / \Gamma \rightarrow X$ is bijective. Let us look at $U \times{ }_{X} U=\left\{\left(u_{1}, u_{2}\right) \mid \pi\left(u_{1}\right)=\pi\left(u_{2}\right)\right\}$. Now the projection on the first factor $p_{1}: U \times{ }_{X} U \rightarrow U$ is again an analytical covering. Since $U$ is simply connected, we must have $p_{1}: Z \stackrel{\sim}{\rightarrow} U$ for every connected component $Z$ of $U \times{ }_{X} U$. The same is true for $p_{2}: U \times{ }_{X} U \rightarrow U$, the projection on the second factor.

Let $(a, b) \in U \times{ }_{X} U$ and let $Z$ be the connected component of $U \times{ }_{X} U$ containing $(a, b)$. Now we have the following commutative diagram :

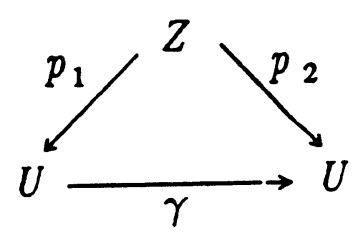


Here $\gamma \in \Gamma$ and $\gamma(a)=b$. This shows that $U / \Gamma \simeq X$.

Since $X$ is proper, there exists a finite covering $\left\{X_{i} \mid i \in I\right\}$ of $X$. We may assume that $\pi^{-1}\left(X_{i}\right)$ is a disjoint union of copies of $X_{i}$. Now the covering $\mathcal{C}=\left\{Y \subseteq K^{2}-\{(0,0)\} \mid Y \in \pi^{-1}\left(X_{i}\right)\right.$ for some $\left.i \in I\right\}$ of $K^{2}-\{(0,0)\}$ shows that $\Gamma$ acts discontinuously and without fixed points.

Lemma 1.3. An analytic automorphism $g$ of $K^{2}-\{(0,0)\}$ can be extended uniquely to an analytic automorphism of $K^{2}$.

Proof. Let $g$ be defined by $g\left(z_{1}, z_{2}\right)=\left(g_{1}\left(z_{1}, z_{2}\right), g_{2}\left(z_{1}, z_{2}\right)\right)$. Let $U=K^{2}-\{(0,0)\}$ be $U=U_{1} \cup U_{2}$ and $U_{1}=K^{*} \times K$ and $U_{2}=K \times K^{*}$. We can expand $g_{1}$ and $g_{2}$ into a convergent power series on $U_{1}$ and $U_{2}$ :

$$
\begin{aligned}
& \left.g_{1}\right|_{U_{1}}=\sum_{m \geq 0} a_{n, m} z_{1}^{n} z_{2}^{m} \\
& \left.g_{1}\right|_{U_{2}}=\sum_{n \geq 0} b_{n, m} z_{1}^{n} z_{2}^{m}
\end{aligned}
$$

These two power series have to be equal on $X_{1} \cap X_{2}$ so we have

$$
\left.g_{1}\right|_{U}=\sum_{n, m \geq 0} a_{n, m} z_{1}^{n} z_{2}^{m} .
$$

This power series of $g_{1}$ is also holomorphic in $(0,0)$. So $g_{1}$ is an analytic function on $K^{2}$. The same is true of $g_{2}$. Therefore we have an unique extension of $g$ to an analytic automorphism of $K^{2}$. It is clear that $g(0,0)=(0,0)$.

Definitions. Let $\left|K^{*}\right|:=\left\{|a| \mid a \in K^{*}\right\}$ be the norm group of $K$. Let $R \in\left|K^{*}\right|$ and $R>1$. We now define :

$$
\begin{aligned}
B_{R} & =\left\{\left(z_{1}, z_{2}\right) \in K^{2} \mid \max \left(\left|z_{1}\right|,\left|z_{2}\right|\right) \leq R\right\} \\
\partial B_{R} & =\left\{\left(z_{1}, z_{2}\right) \in K^{2} \mid \max \left(\left|z_{1}\right|,\left|z_{2}\right|\right)=R\right\}
\end{aligned}
$$

A contraction $\gamma \in \Gamma$ is an automorphism of $K^{2}$ such that :

$$
\gamma\left(\partial B_{R}\right) \subset B_{R}-\partial B_{R}
$$


Lemma 1.4. Let $F \subset K^{2}-\partial B_{R}$ be a connected affinoid subspace. Then either $F \subset K^{2}-B_{R}$ or $F \subset B_{R}-\partial B_{R}$.

Proof. Let $R^{\prime} \in\left|K^{*}\right|, R^{\prime}>R$. We consider the following affinoid subspaces of $K^{2}$ :

$$
\begin{aligned}
& I_{1}=\left\{\left(z_{1}, z_{2}\right) \in K^{2}|| z_{1}|\leq| z_{2} \mid \leq R\right\} \\
& I_{2}=\left\{\left(z_{1}, z_{2}\right) \in K^{2}|| z_{2}|\leq| z_{1} \mid \leq R\right\} \\
& I_{3}=\left\{\left(z_{1}, z_{2}\right) \in K^{2}|| z_{1}|\leq| z_{2}|, R \leq| z_{2} \mid \leq R^{\prime}\right\} \\
& I_{4}=\left\{\left(z_{1}, z_{2}\right) \in K^{2}|| z_{2}|\leq| z_{1}|, R \leq| z_{1} \mid \leq R^{\prime}\right\}
\end{aligned}
$$

For $R^{\prime}>R$ sufficient large we have :

$$
F=F_{1} \cup F_{2} \cup F_{3} \cup F_{4} \quad \text { where } \quad F_{i}:=F \cap I_{i}, i=1 \ldots 4
$$

Because $\left(I_{1} \cup I_{2}\right) \cap\left(I_{3} \cup I_{4}\right) \subseteq \partial B_{R}$ we have $\left(F_{1} \cup F_{2}\right) \cap\left(F_{3} \cup F_{4}\right)=\varnothing$. Since $F$ is connected, either $F_{1} \cup F_{2}=\varnothing$ or $F_{3} \cup F_{4}=\varnothing$. This proves the lemma.

LEMMA 1.5. The group $\Gamma$ contains a contraction $\gamma$.

Proof. The subspace $\partial B_{R} \subset K^{2}$ is the union of the two affinoid subspaces $\left\{\left(z_{1}, z_{2}\right) \in K^{2}|| z_{1}|=R,| z_{2} \mid \leq R\right\}$ and $\left\{\left(z_{1}, z_{2}\right) \in K^{2}|| z_{2}|=R,| z_{1} \mid \leq R\right\}$. The intersection of these two subspaces is connected and non-empty, so $\partial B_{R}$ is connected.

Furthermore since the Hopf surface $X=K^{2}-\{(0,0)\} / \Gamma$ is proper, we know that $\Gamma$ is not finite. Indeed, suppose $\Gamma$ is finite. Now $\mathcal{O}(X)=\mathcal{O}\left(K^{2}-\{(0,0)\}\right)^{\Gamma}$ is not finite dimensional over $K$. Since $\mathcal{O}\left(K^{2}-\{(0,0)\}\right.$ is not finite dimensional. This shows that $X$ cannot be proper (See [BGR] or [Ki.1].).

Since $\Gamma$ is not finite, there exists a $\gamma \in \Gamma$ such that $\gamma\left(\partial B_{R}\right) \cap \partial B_{R}=\varnothing$. Now applying the previous lemma, we have one of the following :

$$
\text { 1) } \begin{aligned}
\gamma\left(\partial B_{R}\right) \subset B_{R}-\partial B_{R} \\
\text { 2) } \gamma\left(\partial B_{R}\right) \subset K^{2}-B_{R}
\end{aligned}
$$

In the first case $\gamma$ is already a contraction, so then the lemma is true. In the second case we have : $B_{R} \cap \gamma\left(\partial B_{R}\right)=\varnothing$. We now apply lemma 1.4 with $F=\gamma^{-1}\left(B_{R}\right)$. So we have : $\gamma^{-1}\left(B_{R}\right) \subset B_{R}-\partial B_{R}$, since $\gamma^{-1}((0,0))=(0,0)$. This proves that $\gamma^{-1} \in \Gamma$ is a contraction. 
Proposition 1.1. The group $\Gamma$ contains a contraction $\gamma$ such that

$$
\Gamma_{0}=\langle\gamma>
$$

is in the centre of $\Gamma$ and $\left[\Gamma: \Gamma_{0}\right]<\infty$.

Proof. Let $\gamma \in \Gamma$ be a contraction defined by

$$
\gamma\left(z_{1}, z_{2}\right)=\left(a\left(z_{1}, z_{2}\right), b\left(z_{1}, z_{2}\right)\right),
$$

where $a\left(z_{1}, z_{2}\right)=\sum_{n+m \geq 1} a_{n, m} z_{1}^{n} z_{2}^{m}$ and $b\left(z_{1}, z_{2}\right)=\sum_{n+m \geq 1} b_{n, m} z_{1}^{n} z_{2}^{m}$.

Since $\gamma\left(\partial B_{R}\right) \subset B_{R}-\partial B_{R}$ we have :

$$
R>\max _{z \in \partial B_{R}}\left|a\left(z_{1}, z_{2}\right)\right|=\max _{z \in \partial B_{R}}\left|\sum_{n+m \geq 1} a_{n, m} z_{1}^{n} z_{2}^{m}\right|=\max \left|a_{n, m}\right| R^{n+m} .
$$

A similar result is true for $b\left(z_{1}, z_{2}\right)$, so we may conclude :

$$
\exists r \in\left|K^{*}\right|, r>R, \gamma\left(B_{R}\right) \subset B_{r} .
$$

The linear part of $\gamma$ has a matrix $\left(\begin{array}{ll}a_{10} & a_{01} \\ b_{10} & b_{01}\end{array}\right)$. All coefficients have an absolute value $<1$. In particular the order of $\gamma$ is not finite. It is clear that if $R^{\prime} \leq R$ then $\gamma\left(B_{R^{\prime}}\right) \subset B_{S}, S:=\frac{R^{\prime}}{R}$, because :

$$
\max _{n+m \geq 1}\left|a_{n, m}\right|\left(R^{\prime}\right)^{n+m}=\max _{n+m \geq 1}\left|a_{n, m}\right| R^{n+m}\left(\frac{R^{\prime}}{R}\right)^{n+m} \leq r \cdot \frac{R^{\prime}}{R} .
$$

If $R^{\prime}>R$ we look at the subspace

$$
Y=\left\{\left(z_{1}, z_{2}\right) \in K^{2} \mid R \leq \max \left(\left|z_{1}\right|,\left|z_{2}\right|\right) \leq R^{\prime}\right\} \subset B_{R^{\prime}} .
$$

The space $Y$ is the union of two affinoid subspaces $Y_{1}$ and $Y_{2}$, where

$$
\begin{gathered}
Y_{1}=\left\{\left(z_{1}, z_{2}\right) \in K^{2}|R \leq| z_{1}\left|\leq R^{\prime},\right| z_{2}|\leq| z_{1} \mid\right\} \\
\text { and } \\
Y_{2}=\left\{\left(z_{1}, z_{2}\right) \in K^{2}|R \leq| z_{2}\left|\leq R^{\prime},\right| z_{1}|\leq| z_{2} \mid\right\} .
\end{gathered}
$$


Since $\gamma$ is not of finite order and $\Gamma$ acts discontinuously on $K^{2}-\{(0,0)\}$, we have :

$$
\exists n>0, \gamma^{n}(Y) \cap Y=\varnothing
$$

In particular we have $: \gamma^{n}(Y) \cap \partial B_{R}=\varnothing$.

Now $Y$ is connected and we may apply lemma 1.4, therefore we have :

$$
\gamma^{n}(Y) \subset K^{2}-B_{R} \text { or } \gamma^{n}(Y) \subset B_{R} .
$$

Since $\gamma^{n}\left(B_{R}\right) \subset B_{R}$ and $B_{R} \cup Y$ is also connected, we must have : $\gamma^{n}\left(B_{R^{\prime}}\right) \subset B_{R}$.

Now we have proved that every point $p \in K^{2}-\{(0,0)\}$ has a $\Gamma_{0}$ - image in the subspace

$$
Z=\left\{\left(z_{1}, z_{2}\right) \in K^{2}-\{(0,0)\} \mid \rho \leq \max \left(\left|z_{1}\right|,\left|z_{2}\right|\right) \leq R\right\} \subseteq K^{2}-\{(0,0)\},
$$

where $\rho<R$ is taken such that $B_{\rho} \subset \gamma\left(B_{R}\right)$. This subspace $Z$ is the union of two affinoid subspace $Z_{1}$ and $Z_{2}$, where

$$
\begin{aligned}
& Z_{1}=\left\{\left(z_{1}, z_{2}\right) \in K^{2}|\rho \leq| z_{1}|\leq R,| z_{2}|\leq| z_{1} \mid\right\} \\
& \quad \text { and } \\
& Z_{2}=\left\{\left(z_{1}, z_{2}\right) \in K^{2}|\rho \leq| z_{2}|\leq R,| z_{1}|\leq| z_{2} \mid\right\} .
\end{aligned}
$$

If $\left[\Gamma: \Gamma_{0}\right]$ were not finite there would be an infinite number of elements $\alpha \in \Gamma$ such that :

$$
\alpha\left(Z_{1}\right) \cap Z_{1} \neq \varnothing, \alpha\left(Z_{2}\right) \cap Z_{2} \neq \varnothing .
$$

Since $\Gamma$ acts discontinously on $K^{2}-\{(0,0)\}$, we must have $\left[\Gamma: \Gamma_{0}\right]$ is finite.

Now we may suppose that $\Gamma_{0} \subset \Gamma$ is a normal subgroup, since we can replace $\Gamma_{0}$ by the intersection of all subgroups conjugated with $\Gamma_{0}$. So for an elements $a \in \Gamma$ we have $a \gamma a^{-1}=\gamma^{n}$ for some $n \in \mathbb{Z}$. The linear part of $\gamma$ has eigenvalues with absolute value $<1$. This shows that only $a \gamma a^{-1}=\gamma$ can occur. This proves that $\Gamma_{0}$ is in the centre of $\Gamma$.

THEOREM 1.1. There exist global parameters $t_{1}, t_{2}$ of $K^{2}$ such that a contraction $\gamma$ has the following form :

$$
\gamma\left(t_{1}, t_{2}\right)=\left(\alpha_{1} t_{1}+\lambda t_{2}^{m}, \alpha_{2} t_{2}\right) .
$$

Here $0<\left|\alpha_{1}\right| \leq\left|\alpha_{2}\right|<1$ and $\lambda=0$ if $\alpha_{1} \neq \alpha_{2}^{m}$ otherwise $\lambda \in K$.

Proof. This will be proved in the following three lemmas. 
Lemma 1.6. There exist formal parameters $t_{1}, t_{2} \in K \llbracket z_{1}, z_{2} \rrbracket$ such that $\gamma\left(t_{1}, t_{2}\right)$ has the form described in theorem 1.1 above.

Proof. It is clear that $\gamma$ also acts on the formal local ring $K \llbracket z_{1}, z_{2} \rrbracket$. We denote this action with $\tilde{\gamma}$. Let $\underline{m}, \underline{m}^{2}, \underline{m}^{3}, \ldots$ be the powers of the maximal ideal $\underline{m}$ of $K \llbracket z_{1}, z_{2} \rrbracket$.

Now, after possibly an extension of degree two of $K, \tilde{\gamma}$ has two eigenvalues $\alpha_{1}, \alpha_{2}$ on $\underline{m} / \underline{m}^{2}$. Let us take $\left|\alpha_{1}\right| \leq\left|\alpha_{2}\right|$. We can make a linear transformation of the variables $z_{1}$ and $z_{2}$ such that the linear part of $\gamma$, which is the matrix of $\tilde{\gamma}$ on $\underline{m} / \underline{m}^{2}$, has the form $\left(\begin{array}{cc}\alpha_{1} & * \\ 0 & \alpha_{2}\end{array}\right)$ and $* \neq 0$ only if $\alpha_{1}=\alpha_{2}$.

With respect to this basis $z_{1}, z_{2}, z_{1}^{2}, z_{1} z_{2}, z_{2}^{2}, \ldots, z_{2}^{n}$ of $\underline{m} / \underline{m}^{n+1}, n \geq 1$ we find $\tilde{\gamma}$ has a triangular matrix :

$$
\left(\begin{array}{ccccc}
\alpha_{1} & & & & \\
& \alpha_{2} & & * & \\
& & \alpha_{1}^{2} & & \\
& 0 & & \ddots & \\
& & & & \alpha_{2}^{n}
\end{array}\right)
$$

If $\alpha_{1} \neq \alpha_{2}$ then the eigenvalue $\alpha_{2}$ only occurs once. So modulo $\underline{m}^{n+1}$ there is an unique one-dimensional eigenspace belonging to $\alpha_{2}$. Taking the limit $n \rightarrow \infty$ we get an unique formal power series $t_{2}=z_{2}+\ldots$ such that $\tilde{\gamma}\left(t_{2}\right)=\alpha_{2} t_{2}$.

If $\alpha_{1}=\alpha_{2}$ then the value eigenvalue $\alpha_{2}$ occurs twice. So modulo $\underline{m^{n+1}}$ there is an unique two-dimensional eigenspace belonging to $\alpha_{2}$. Taking the limit $n \rightarrow \infty$ we can find in this eigenspace a formal power series $t_{2}=z_{2}+\ldots$ such that $\tilde{\gamma}\left(t_{2}\right)=\alpha_{2} t_{2}$.

If $\alpha_{1} \neq \alpha_{2}^{m} \forall m \geq 1$ then also the eigenvalue $\alpha_{1}$ occurs only once. Again we find an unique one-dimensional eigenspace belonging to $\alpha_{1}$ and a power series $t_{1}=z_{1}+\ldots \in K \llbracket z_{1}, z_{2} \rrbracket$ such that $\tilde{\gamma}\left(t_{1}\right)=\alpha_{1} t_{1}$.

If $\alpha_{1}=\alpha_{2}^{m}$ for a certain $m \geq 1$ then the eigenvalue $\alpha_{1}$ always occurs twice for $n \geq m \geq 1$. It has also an eigenvector $t_{2}^{m}$. Now we can find a power series $t_{1} \in K \llbracket z_{1}, z_{2} \rrbracket$ such that $\tilde{\gamma}\left(t_{1}\right)=\alpha_{1} t_{1}+\lambda t_{2}^{m}$. This $t_{1}$ is not unique, we could also have taken $t_{1}+\mu t_{2}^{m}, \mu \in K$. This proves the lemma for some formal parameters $t_{1}, t_{2}$ in $(0,0)$. 
Lemma 1.7. The parameters $t_{1}$ and $t_{2}$ constructed in lemma 1.6 are holomorphic functions on $K^{2}$.

Proof. Let us choose an $R \in\left|K^{*}\right|, R \gg 0$. Let

$$
V=\left\{f \in \mathcal{O}\left(B_{R}\right) \mid f(0,0)=0\right\}
$$

be the Banach space of functions that are holomorphic on $B_{R}$. On $V$ we have the sup-norm.

The contraction $\gamma$ induces an action $\tilde{\gamma}: V \rightarrow V$ on $V$. In the proof of proposition 1.1 we have shown that $\exists r \in\left|K^{*}\right| r<R \gamma\left(B_{R}\right) \subset B_{r}$. Since $\gamma\left(B_{R}\right) \subset B_{r} \subset B_{R}$, the operator $\tilde{\gamma}$ acting on $V$ is compact. The $p$-adic theory of compact operators (See [G]) tells us that for every $\lambda \in K^{*}$ we have :

1) $K_{\lambda}=\bigcup_{n \geq 1} \operatorname{ker}\left((\tilde{\gamma}-\lambda)^{n}: V \rightarrow V\right)$ is finite dimensional

2) $K_{\lambda}$ has a $\tilde{\gamma}$-invariant closed complement $W_{\lambda}$ in $V$ and $(\tilde{\gamma}-\lambda): W_{\lambda} \tilde{\rightarrow} W_{\lambda}$

So we can suppose $V=K_{\lambda} \oplus W_{\lambda}$ for somme $\lambda \in K^{*}$. Furthemore we have $V /\left(z_{1}, z_{2}\right)^{n} V \simeq \underline{m} / \underline{m}^{n+1}$, where $\underline{m}$ is the maximal ideal of $K \llbracket z_{1}, z_{2} \rrbracket$. As in the previous lemma this shows that the eigenspace $K_{\lambda}$ for $\lambda=\alpha_{1}$ or $\lambda=\alpha_{2}$ has dimension 1 or 2 . Specially the parameters $t_{1}$ and $t_{2}$ of lemma 1.6 are in fact holomorphic functions, since they are holomorphic on any $B_{R}$, $R \gg 0, R \in\left|K^{*}\right|$.

Lemma 1.8. The map $t: K^{2} \rightarrow K^{2}$ defined by $t\left(z_{1}, z_{2}\right)=\left(t_{1}, t_{2}\right)$ is invertible, so $t_{1}, t_{2}$ are global parameters of $K^{2}$ and $t: K^{2} \rightarrow K^{2}$ is an isomorphism.

Proof. Let $\rho \in\left|K^{*}\right|$ be sufficiently small. Then the map

$$
t: B_{\rho}=\left\{\left(z_{1}, z_{2}\right) \in K^{2} \mid \max \left(\left|z_{1}\right|,\left|z_{2}\right|\right) \leq \rho\right\} \rightarrow B_{\rho}
$$

is an isomorphism. This can be seen by considering the linear part of $t$. Let $s_{0}$ be the inverse of $t$. Let $\delta$ be the transformation on the second $B_{\rho}$ defined by $\delta\left(a_{1}, a_{2}\right)=\left(\alpha_{1} a_{1}+\lambda a_{2}^{m}, \alpha_{2} a_{2}\right)$. It is clear that $t \circ \gamma=\delta \circ t$.

For every $R \in\left|K^{*}\right|, R>\rho$ there exists an $n \geq 1$ such that $\delta^{n}\left(B_{R}\right) \subset B_{\rho}$. Let $s$ be $s: B_{R} \stackrel{\delta^{n}}{\rightarrow} B_{\rho} \stackrel{s_{0}}{\rightarrow} B_{\rho} \stackrel{\gamma^{-n}}{\rightarrow} K^{2}$, so $s=\gamma^{-n} \circ s_{0} \circ \delta^{n}$. 
Now we have :

$$
\begin{aligned}
& t \circ s=t \gamma^{-n} s_{0} \delta^{n}=\delta^{-1} t \gamma \gamma^{-n} s_{0} \delta^{n}=\delta^{-n} t s_{0} \delta^{n}=\delta^{-n} \delta^{n}=1 \text { and } \\
& s \circ t=\gamma^{-n} s_{0} \delta^{n} t=\gamma^{-n} s_{0} \delta^{n-1} t \gamma=\gamma^{-n} s_{0} t \gamma^{n}=\gamma^{-n} \gamma^{n}=1
\end{aligned}
$$

So the maps $s$ do not depend on the choice of $n$. We can glue them together into a map $s: K^{2} \rightarrow K^{2}$. Of course $s \circ t=t \circ s=i d$, since the germ of $s \circ t$ and $t \circ s$ in $(0,0)$ is the identity map.

Remark. Another way to prove the previous lemma would be the following. The map $t: K^{2}-\{(0,0)\} \rightarrow K^{2}-\{(0,0)\}$ is already invertable on a small polydisc $B_{\rho}-\{(0,0)\}$ around $(0,0)$. This gives an isomorphism $\varphi$ :

$$
\begin{aligned}
& K^{2}-\{(0,0)\} /<\gamma>\underset{\varphi}{\underset{\varphi^{. j}}{\longrightarrow}} K^{2}-\{(0,0)\} /<\delta> \\
& \begin{array}{ccc}
\uparrow & & \uparrow \\
K^{2}-\{(0,0)\} & \stackrel{t}{\rightleftarrows} & K^{2}-\{(0,0)\}
\end{array}
\end{aligned}
$$

Since $K^{2}-\{(0,0)\}$ is simply connected (lemma 1.1), there exists a lifting $L$ of $\varphi^{-1}$. We can choose the lifting $L$ such that $L \circ t=t \circ L=1$.

THEOREM 1.2. The group $\Gamma$ is abelian and $\Gamma \cong \mathbb{Z} \times \mathbb{Z} / l \mathbb{Z}$.

Proof. Let $\gamma \in \Gamma$ be a contraction lying in the centre of $\Gamma$.

First we look at the case where $\gamma\left(z_{1}, z_{2}\right)=\left(\alpha z_{1}, \alpha z_{2}\right), 0<|\alpha|<1$.

Now we have :

$$
\delta \in \Gamma \Longrightarrow \delta \gamma=\gamma \delta \Longrightarrow \delta\left(\alpha z_{1}, \alpha z_{2}\right)=\alpha . \delta\left(z_{1}, z_{2}\right)
$$

So $\delta$ is linear, $\delta\left(z_{1}, z_{2}\right)=\left(\beta_{1} z_{1}+\lambda z_{2}, \beta_{2} z_{2}\right)$ for a suitable choice of coordinates.

If $\exists \delta \in \Gamma$ with $\beta_{1}=1$ then $\delta(z, 0)=(z, 0)$. Since $\Gamma$ acts without fixed points, we have $\delta=1$. Therefore the map $\varphi: \Gamma \rightarrow K$ defined by $\varphi(\delta)=\beta_{1}$-coordinate is injective. Now we can conclude that $\Gamma$ is abelian. Since $\Gamma$ acts discontinuouly we must have $\Gamma \cong \mathbb{Z} \times \Gamma_{\text {torsion }}$ and $\mathbb{Z} \subset \Gamma$ is generated by a contraction. The injectivity of $\varphi$ shows that $\Gamma_{\text {torsion }} \cong \mathbb{Z} / l \mathbb{Z}$ for some $l \in \mathbb{Z}_{\geq 1}$. Now we look at the case where

$$
\gamma\left(z_{1}, z_{2}\right)=\left(\alpha_{1} z_{1}, \alpha_{2} z_{2}\right), 0<\left|\alpha_{1}\right| \leq\left|\alpha_{2}\right|<1, \alpha_{1} \neq \alpha_{2}
$$


The eigenspace belonging to $\alpha_{1}$ is :

a) 1-dimensional if $\alpha_{1} \neq \alpha_{2}^{m}, \forall m \geq 1$ or

b) 2-dimensional if $\exists m \in \mathbb{Z}_{>1}, \alpha_{1}=\alpha_{2}^{m}$.

In case $a$ we have :

$$
\delta \in \Gamma \Longrightarrow \delta \gamma=\gamma \delta \Longrightarrow \delta\left(z_{1}, z_{2}\right)=\left(\beta_{1} z_{1}, \beta_{2} z_{2}\right)
$$

This shows that $\Gamma$ has to be abelian. Since $\Gamma$ acts discontinuously, we must have $\Gamma \cong \mathbb{Z} \times \Gamma_{\text {torsion }}$ and $\mathbb{Z} \subset \Gamma$ is generated by a contraction. Now since the element $\delta:\left(z_{1}, z_{2}\right) \rightarrow\left(\beta_{1} z_{1}, \beta_{2} z_{2}\right)$ is fixed point free we must have $\Gamma_{\text {torsion }} \simeq \mathbb{Z} / l \mathbb{Z}$. Clearly $\Gamma_{\text {torsion }}$ is generated by $\tilde{\omega}:\left(z_{1}, z_{2}\right) \rightarrow\left(\omega^{i} z_{1}, \omega z_{2}\right), \omega^{l}=1$ and g.c.d. $(i, l)=1$.

In case $b$ we have :

$$
\delta \in \Gamma \Longrightarrow \delta \gamma=\gamma \delta \Longrightarrow \delta\left(z_{1}, z_{2}\right)=\left(\beta_{1} z_{1}+\mu z_{2}^{m}, \beta_{2} z_{2}\right)
$$

Again the map $\varphi: \Gamma \rightarrow K$ defined by $\varphi(\delta)=\beta_{1}$ - coordinate is injective. Therefore $\Gamma$ is abelian and we have $\Gamma \simeq \mathbb{Z} \times \mathbb{Z} / l \mathbb{Z}$.

Now we consider the case where $\gamma\left(z_{1}, z_{2}\right)=\left(\alpha_{1} z_{1}+\lambda z_{2}^{m}, \alpha_{2} z_{2}\right), \alpha_{2}^{m}=\alpha_{1}$.

Let $\delta \in \Gamma$, then we have :

$$
\delta \gamma=\gamma \delta \Longrightarrow \delta\left(z_{1}, z_{2}\right)=\left(\beta_{1} z_{1}+\mu z_{2}^{m}, \beta_{2} z_{2}\right), \beta_{1}=\beta_{2}^{m}
$$

Again $\Gamma$ is abelian and therefore $: \Gamma \simeq \mathbb{Z} \times \mathbb{Z} / l \mathbb{Z}$.

Remark. We can also describe the generator of the torsion subgroup explicitly when the group $\mathbb{Z} \subset \Gamma$ is generated by a contraction $\gamma$ of the form :

$$
\gamma\left(z_{1}, z_{2}\right)=\left(\alpha_{1} z_{1}+\lambda z_{2}^{m}, \alpha_{2} z_{2}\right), \alpha_{2}^{m}=\alpha_{1}
$$

Let $\tilde{\omega}$ be a generator of $\mathbb{Z} / l \mathbb{Z}$. Then $\tilde{\omega}$ has the following form :

$$
\tilde{\omega}:\left(z_{1}, z_{2}\right) \rightarrow\left(\omega\left(z_{1}+\mu z_{2}^{m}\right), \omega^{j} z_{2}\right), \omega^{l}=1,(l, j)=1 .
$$

Now we have :

$$
\begin{aligned}
\tilde{\omega}^{l}:\left(z_{1}, z_{2}\right)=\left(\omega^{l} z_{1}+\left(\omega^{l}+\omega^{l-1} \omega^{j m}+\omega^{l-2} \omega^{2 j m}+\cdots\right.\right. \\
\\
\left.\left.+\omega \omega^{(l-1) j m}\right) \mu z_{2}^{m}, \omega^{j m l} z_{2}\right)
\end{aligned}
$$


Since $\tilde{\omega}^{l}=1$ we must have :

$$
\begin{aligned}
& 0=\mu \sum_{k=1}^{l} \omega^{k} \omega^{(l-k) j m}=\mu \omega^{j m l} \sum_{k=1}^{l} \omega^{k(1-j m)}=\mu \sum_{k=1}^{l} \omega^{k(1-j m)} \\
& \Leftrightarrow \mu=0 \vee \sum_{k=1}^{l} \omega^{(1-j m) k}=0
\end{aligned}
$$

Now $\omega$ is a primitive $l-t h$ root of unity, so we have :

$$
\sum_{k=1}^{l} \omega^{k(1-j m)}= \begin{cases}l & \text { if } j m \equiv 1 \bmod l \\ 0 & \text { otherwise }\end{cases}
$$

So there are no restrictions on $\tilde{\omega}$ when $m j \neq 1 \bmod l$. When $j m=1$ $\bmod l$ then of course $(l, m)=1$ and $\mu=0$ because $l \equiv 0$ cannot occur (when $\operatorname{char}(K)=p>0$ there are no $p$-th roots of unity $\neq 1$ ).

Since $\tilde{\omega}$ has to commute with $\gamma$, we have :

$$
\tilde{\omega} \delta=\delta \tilde{\omega} \Leftrightarrow \lambda=0 \vee j m=1 \bmod l \text {. }
$$

This gives us all the possibilities for $\tilde{\omega}$ :

$$
\begin{aligned}
& \lambda \neq 0 \Longrightarrow j m=1 \bmod l,(l, m)=1, \mu=0 \\
& \lambda=0 \Longrightarrow j m \neq 1 \bmod l, \mu \in K \text { or } j m=1 \bmod l,(l, m)=1, \mu=0 .
\end{aligned}
$$

ThEOREM 1.3. Let $\Gamma$ be generated by a contraction $\gamma$ and let $X$ be the Hopf surface $K^{2}-\{(0,0)\} / \Gamma$. Then the field $\mathcal{M}(X)$ of meromorphic functions on $X$ is :

$$
\begin{array}{r}
\text { 1) } K\left(\frac{z_{1}^{a}}{z_{2}^{b}}\right), \text { if } \gamma\left(z_{1} \cdot z_{2}\right)=\left(\alpha_{1} z_{1}, \alpha_{2} z_{2}\right), \alpha_{1}^{a}=\alpha_{2}^{b}, g . c . d .(a, b)=1, \\
a, b \in \mathbb{Z}_{>0} . \\
\text { 2) } K\left(\frac{z_{1}^{p}-\lambda^{p-1} z_{1} z_{2}^{m p-m}}{z_{2}^{m}}\right), \text { if } \gamma\left(z_{1}, z_{2}\right)=\left(\alpha_{1} z_{1}+\lambda z_{2}^{m}, \alpha_{2} z_{2}\right), \\
\alpha_{2}^{m}=\alpha_{1}, \lambda \neq 0, \operatorname{char}(K)=p>0 .
\end{array}
$$

3) $K$ in all other cases.

Proof. We have the following identities :

$$
\begin{aligned}
\mathcal{M}(X) & =\left\{f \mid f \text { is meromorphic on } K^{2}-\{(0,0)\} \text { and } \gamma \text {-invariant }\right\} \\
& =\left\{f \mid f \text { is meromorphic on } K^{2} \text { and } \gamma \text {-invariant }\right\} .
\end{aligned}
$$


Since $K^{2}$ is a quasi-Stein space, we can now write : $f=\frac{t}{s}, t \in \mathcal{O}\left(K^{2}\right)$. (The proof of this fact is the same as the one given in [FP] theorem VI.3.5 for $\left.\left(K^{*}\right)^{n}\right)$. We can choose $t, s$ in such a way that they are minimal, i.e. have only a finite number of zeroes in common. Let

$$
t=\sum_{n, m \geq 0} a_{n, m} z_{1}^{n} z_{2}^{m} \in \mathcal{O}\left(K^{2}\right) \text { and } s=\sum_{n, m \geq 0} b_{n, m} z_{1}^{n} z_{2}^{m} \in \mathcal{O}\left(K^{2}\right) .
$$

Let us first consider the case where $\gamma$ has the form : $\gamma\left(z_{1}, z_{2}\right)=\left(\alpha_{1} z_{1}, \alpha_{2} z_{2}\right)$, $0<\left|\alpha_{1}\right| \leq\left|\alpha_{2}\right|<1$. Then clearly we have : $\gamma(t)=c t \Longrightarrow c=\alpha_{1}^{k} \alpha_{2}^{l}$ for some $k, l \in \mathbb{Z}_{\geq 0}$. that:

Now suppose that $\alpha_{1}^{a} \neq \alpha_{2}^{b} \forall a, b \in \mathbb{Z}$ and $(a, b) \neq(0,0)$. Then it is clear

$$
\begin{gathered}
\gamma(f)=f \Rightarrow \gamma(t)=c t \wedge \gamma(s)=c s \Rightarrow t=\lambda z_{1}^{k} z_{2}^{l} \wedge s=\mu z_{1}^{k} z_{2}^{l} \\
\Rightarrow f=\frac{t}{s} \in K .
\end{gathered}
$$

So we have : $\mathcal{M}(X)=K$.

Next we suppose that $\alpha_{1}^{a}=\alpha_{2}^{b}$ for some $a, b \in \mathbb{Z}_{\geq 0},(a, b) \neq(0,0)$. We can choose $a, b$ minimal, such that g.d.c. $(a, b)=1$. Then we have $\alpha_{1}^{d}=\alpha_{2}^{c} \Longrightarrow(d, c)=n(a, b) \quad$ for some $n \in \mathbb{Z}$. Now a monomial $z_{1}^{k} z_{2}^{l}$ with $\gamma\left(z_{1}^{k}, z_{2}^{l}\right)=c z_{1}^{k} z_{2}^{l}$ for a fixed $c=\alpha_{1}^{k_{0}} \alpha_{2}^{l_{0}}$ is of the form $z_{1}^{k} z_{2}^{l}$ with $(k, l)=\left(k_{0}, l_{0}\right)+n(a,-b)$ for some $n \in \mathbb{Z}$. This shows that :

$$
\gamma(f)=f \Rightarrow \gamma(t)=c t \wedge \gamma(s)=c s \Rightarrow \frac{t}{s} \in K\left(\frac{z_{1}^{a}}{z_{2}^{b}}\right) \Rightarrow \mathcal{M}(X)=K\left(\frac{z_{1}^{a}}{z_{2}^{b}}\right) .
$$

Let us now consider the case where $\gamma$ has the form :

$$
\gamma\left(z_{1}, z_{2}\right)=\left(\alpha_{1} z_{1}+\lambda z_{2}^{m}, \alpha_{2} z_{2}\right), \alpha_{2}^{m}=\alpha_{1}, \lambda \neq 0
$$

We can replace $z_{1}$ by $\lambda^{-1} z_{1}$, then $\gamma$ has the form :

$$
\gamma\left(z_{1}, z_{2}\right)=\left(\alpha_{1} z_{1}+z_{2}^{m}, \alpha_{2} z_{2}\right), \alpha_{2}^{m}=\alpha_{1} .
$$

Every monomial $z_{1}^{k} z_{2}^{l}$ can be written as $\left(\frac{z_{1}}{z_{2}^{m}}\right)^{k} z_{2}^{l+k m}$. Let us take $x:=\frac{z_{1}}{z_{2}^{m}}$ and $z_{2}$ as new variables. Then we have :

$$
\gamma(x)=x+1, \gamma\left(z_{2}\right)=\alpha_{2} z_{2}
$$


Let $g$ be polynomial in the variables $x$ and $z_{2}$ with $\gamma(g)=c \cdot g$. Then we clearly have $c=\alpha_{2}^{k}$ for some $K \in \mathbb{Z}_{\geq 0}$. This shows that $g=z_{2}^{k} h$, where $h$ is a polynomial in $x$ with $\gamma(h)=h$. Let us take $h=\sum_{i=0}^{s} a_{i} x^{i}$ and let $s$ be the highest power of $x$ such that $a_{s} \neq 0$. Then we have:

$$
\begin{aligned}
\gamma(h)=h & \Longrightarrow \sum_{i=0}^{s} a_{i} x^{i}=\sum_{i=0}^{s} a_{i}(x+1)^{i} \\
& \Longrightarrow s a_{s}+a_{s-1}=a_{s-1} \\
& \Longrightarrow s=0 \vee a_{s}=0 .
\end{aligned}
$$

Since $a_{s} \neq 0$,we must have $s=0$. When $\operatorname{char}(K)=0$ then $s=0$ and $h \in K$. So in this case $\mathcal{M}(X)=K$.

But when $\operatorname{char}(K)=p>0$, then we see $p \mid s$. Now we look at the polynomial $x^{p}-x$. We have : $\gamma\left(x^{p}-x\right)=(x+1)^{p}-(x+1)=x^{p}-x$.

So any polynomial of the form $\sum a_{i}\left(x^{p}-x\right)^{i}$ is $\gamma-$ invariant. The proof given above also shows that polynomials $\left(x^{p}-x\right)^{i}$ form a basis of the $\gamma-$ invariant polynomials. This shows that :

$$
\mathcal{M}(X)=K\left(x^{p}-x\right)=K\left(\frac{z_{1}^{p}}{z_{2}^{m p}}-\frac{z_{1}}{z_{2}^{m}}\right)=K\left(\frac{z_{1}^{p}-z_{1} z_{2}^{m p-m}}{z_{2}^{m p}}\right) .
$$

\section{Affinoid coverings and reductions}

We first contruct a fundamental domain for the action of the group $\Gamma$, where $\Gamma$ is generated by a contraction. Then we will study some special affinoid subspaces of $K^{2}$ and their reduction. We will use this to contruct a pure covering of $K^{2}-\{(0,0)\}$, which is $\Gamma$ - invariant. This will give us a pure affinoid covering of the Hopf surface $X=K^{2}-\{(0,0)\} / \Gamma$.

Definition. We call a subspace $F \subset K^{2}-\{(0,0)\}$ a fundamental domain for the action of the group $\Gamma$, if $F$ has the following properties :

1) $K^{2}-\{(0,0)\}=\bigcup_{\gamma \in \Gamma} \gamma(F)$.

2) There exists a finite affinoid covering $\left\{S p\left(A_{i}\right)\right\}_{i=1}^{n}$ of $F$.

3) The only action of $\Gamma$ on $F$ itself is the identification of a finite number of affinoid subspaces $B_{k} \subset F$, where $B_{k} \subset S p\left(A_{i}\right)$ is defined by a finite number $s$ of equations :

$$
\left|f_{j}\right|=c_{j}, j=1 \ldots s, f_{j} \in A_{i}, c_{j} \in\left|K^{*}\right| .
$$


So the subspaces $B_{k}$ are of the form

$$
B_{k}=S p\left(A_{i}<\frac{f_{i}}{c_{j}}, \frac{c_{j}}{f_{j}} \mid j=1 \ldots s>\right) .
$$

Proposition 2.1. Let $\Gamma$ be generated by a contraction

$$
\gamma:\left(z_{1}, z_{2}\right) \rightarrow\left(\alpha_{1}\left(z_{1}+\lambda z_{2}^{m}\right), \alpha_{2} z_{2}\right)
$$

where $0<\left|\alpha_{1}\right| \leq\left|\alpha_{2}\right|<1$ and $\lambda=0$ if $\alpha_{1} \neq \alpha_{2}^{m}$, otherwise $\lambda \in K$. If we choose $|\lambda|<1$ then $\Gamma$ has a fundamental domain $F$ defined by :

$F:=\left\{\left(z_{1}, z_{2}\right) \in K^{2}-\{(0,0)\}|| z_{1}|\leq 1,| z_{2} \mid \leq 1,\left(\left|z_{1}\right| \geq\left|\alpha_{1}\right| \vee\left|z_{2}\right| \geq\left|\alpha_{2}\right|\right)\right\}$

Proof. Let us first show that we may choose $|\lambda|<1$. We can replace the coordinate $z_{1}$ by $\varepsilon z_{1}, \varepsilon \in K^{*}$. Then $\gamma$ is defined by :

$$
\gamma\left(\varepsilon z_{1}, z_{2}\right)=\left(\alpha_{1}\left(\varepsilon z_{1}+\varepsilon \lambda z_{2}^{m}\right), \alpha_{2} z_{2}\right), \lambda \neq 0
$$

Now take $\varepsilon=\mu \lambda^{-1},|\mu|<1$. This gives us the desired form of $\gamma$ :

$$
\gamma\left(z_{1}, z_{2}\right)=\left(\alpha_{1}\left(z_{1}+\mu z_{2}^{m}\right), \alpha_{2} z_{2}\right),|\mu|<1 .
$$

A straightforward calculation now shows that :

$$
\begin{aligned}
& \bigcup_{i \in \mathbb{Z}^{\prime}} \gamma^{i}(F)=K^{2}-\{(0,0)\}, \\
& \gamma^{i}(F) \cap \gamma^{i+1}(F)=\left\{\begin{array}{l}
\left(z_{1}, z_{2}\right) \in K^{2}-\left.\{(0,0)\}|| z_{2}|=| \alpha_{2}\right|^{i} \wedge\left|z_{1}\right| \leq\left|\alpha_{1}\right|^{i} \\
\text { or }\left|z_{1}\right|=\left|\alpha_{1}\right|^{i} \wedge\left|z_{2}\right| \leq\left|\alpha_{2}\right|^{i}
\end{array}\right\}, \\
& \gamma^{i}(F) \cap \gamma^{j}(F)=\varnothing \text { if }|i-j| \neq 0,
\end{aligned}
$$

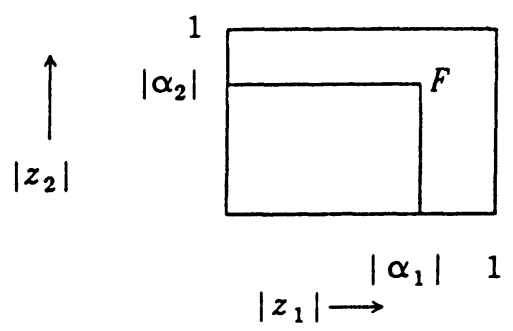


This shows that the subspace $F$ satisfies the first property of our definition of a fundamental domain.

The only action of $\Gamma$ on $F$ is the indentification of $\gamma^{-1}(F) \cap F$ and $F \cap \gamma(F)$. This gives the following indentifications of affinoid subspaces of $F$ :

$$
\gamma\left(C_{1}\right)=C_{2}, \gamma\left(C_{3}\right)=C_{4} .
$$

Here $C_{i}, i=1 \ldots 4$ are defined by :

$$
\begin{aligned}
& C_{1}=\left\{\left(z_{1}, z_{2}\right)|| z_{1}|=1,| z_{2} \mid \leq 1\right\} \\
& C_{2}=\left\{\left(z_{1}, z_{2}\right)|| z_{1}|=| \alpha_{1}|,| z_{2}|\leq| \alpha_{2} \mid\right\} \\
& C_{3}=\left\{\left(z_{1}, z_{2}\right)|| z_{2}|=1,| z_{1} \mid \leq 1\right\} \\
& C_{4}=\left\{\left(z_{1}, z_{2}\right)|| z_{2}|=| \alpha_{2}|,| z_{1}|\leq| \alpha_{1} \mid\right\} .
\end{aligned}
$$

We will now show that $F$ can be covered by a finite number of affinoid subspaces, such that $C_{i}, i=1 . .4$ satisfy property 3 of our definition.

If $\left|\alpha_{1}\right|^{k}=\left|\alpha_{2}\right|^{l}, \lambda=0, k, l \in \mathbb{Z}_{>0}$ then $\Gamma$ also preserves the area given by $\left\{\left(z_{1}, z_{2}\right) \in K^{2}-\{(0,0)\}|| \frac{z_{1}^{k}}{z_{2}^{l}} \mid=1\right\}$. This gives a $\gamma$-invariant partition of the domain $F$ into two affinoid subspaces $F_{1}$ and $F_{2}$.

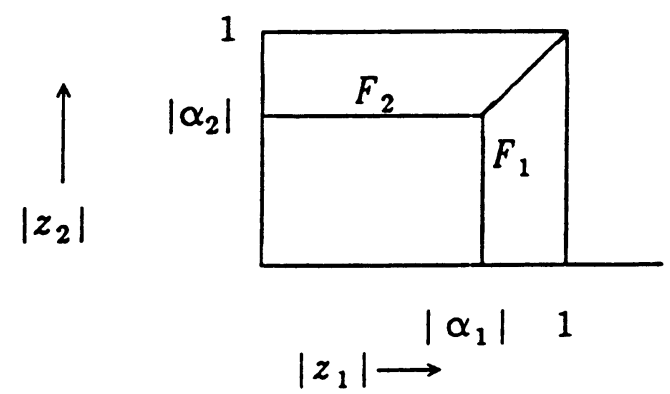

We have :

$$
\begin{aligned}
& F_{1}=\left\{\left(z_{1}, z_{2}\right) \in K^{2}-\{(0,0)\}|| \alpha_{1}|\leq| z_{1}|\leq 1,| z_{2}^{l}|\leq| z_{1}^{k} \mid\right\} \\
& F_{2}=\left\{\left(z_{1}, z_{2}\right) \in K^{2}-\{(0,0)\}|| \alpha_{2}|\leq| z_{2}|\leq 1,| z_{2}^{l}|\geq| z_{1}^{k} \mid\right\} .
\end{aligned}
$$

The affinoid subspases $C_{1}, C_{2} \subset F_{1}$ are defined by $\left|z_{1}\right|=1$ and $\left|z_{1}\right|=$ $\left|\alpha_{2}\right|$ respectively. The subspaces $C_{3}, C_{4} \subset F_{2}$ are defined by $\left|z_{2}\right|=1$ and $\left|z_{2}\right|=\left|\alpha_{1}\right|$ respectj rely. This shows that $F$ is a fundamental domain. 
If $\gamma$ has the form $\gamma\left(z_{1}, z_{2}\right)=\left(\alpha_{1}\left(z_{1}+\lambda z_{2}^{m}\right), \alpha_{2} z_{2}\right),|\lambda|<1$, then again $F=F_{1} \cup F_{2}$ as above with $k=1, l=m$. Since $|\lambda|<1$ the area $\left\{\left(z_{1}, z_{2}\right) \in K^{2}-\{(0,0)\} .|| \frac{z_{1}}{z_{2}^{m}} \mid=1\right\}$ is $\Gamma$ - invariant. Again $F$ is a fundamental domain.

If $\left|\alpha_{1}^{k}\right| \neq\left|\alpha_{2}^{l}\right|, \forall k, l \in \mathbb{Z}_{>0}$ then we can find an $s \in \mathbb{Z}_{>0}$ such that $\left|\alpha_{2}^{s}\right|<\left|\alpha_{1}\right|$ since $0<\left|\alpha_{1}\right|<\left|\alpha_{2}\right|<1$. Now the areas defined by $\left|\frac{z_{1}}{z_{2}^{s}}\right|=1$ and by $\left|\frac{z_{1}}{z_{2}}\right|=\left|\frac{\alpha_{1}}{\alpha_{2}}\right|$ have a non-empty intersection $P$ in $F$. Here $P$ is defined by $\left|z_{2}^{s-1}\right|=\left|\frac{\alpha_{1}}{\alpha_{2}}\right|,\left|z_{1}^{s-1}\right|=\left|\frac{\alpha_{1}}{\alpha_{2}}\right|$. This gives us a finite affinoid covering of $F$ by $F_{1}, F_{2}, F_{3}$ and $F_{4}$ (See figure below). The subspaces $C_{i} \subset F_{i}, i=1 . .4$ have property 2 of our definition. So $F$ is a fundamental domain.

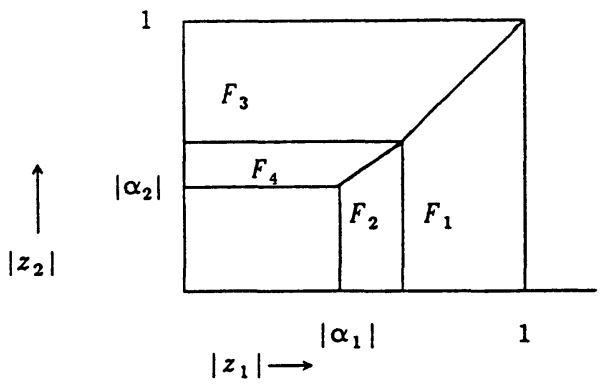

Definitions. Let $A$ be an affinoid algebra and $S p(A)$ its affinoid space. A subspace $X \subset S p(A)$ is called a rational domain if there exists a set $\left\{f_{0}, f_{1}, \ldots f_{n}\right\}$ generating the unit ideal of $A$ such that $X$ is defined by :

$$
\begin{aligned}
X & =\left\{x \in S p(A)|| f_{i}(x)|\leq| f_{0}(x) \mid, i=1 . . n\right\} \\
& =\left\{x \in S p(A)|| \frac{f_{i}(x)}{f_{0}(x)} \mid \leq 1, i=1 . . n\right\} .
\end{aligned}
$$

The rational domain $X$ is an affinoid subspace of $S p(A)$ and has as its affinoid algebra $A<\frac{f_{i}}{f_{0}}|i=1 . . n\rangle \cong A<x_{1} . . x_{n}>/<f_{0} x_{i}-f_{i}|i=1 . . n\rangle$ (See $[\mathrm{BGR}]$ or $[\mathrm{FP}]$.).

We will only use rational subspaces of

$$
Y=S p\left(K<z_{1}, z_{2}>\right) \cong\left\{\left(z_{1}, z_{2}\right) \in K^{2}|| z_{1}|\leq 1,| z_{2} \mid \leq 1\right\} .
$$


In particular we will restrict ourselves to those rational subspaces $X \subset Y$ where the $f_{i}, i=0 . . n$, are monomials $c z_{1}^{k} z_{2}^{l}, k, l \in \mathbb{Z}_{\geq 0}, c \in K^{*}$. Such a subspace $X \subset Y$ will be called a monomial rational subspace (of $Y$ with respect to the affinoid generating set $\left\{z_{1}, z_{2}\right\}$ ).

Example. The affinoid covering of the fundamental domain $F$ constructed in the proof of proposition 2.1 consists of a finite number of monomial rational sbuspaces of $Y$.

We will only show this for the affinoid space, $F_{1}$ when $\left|\alpha_{1}^{k}\right|=\left|\alpha_{2}^{l}\right|$ for some $k, l \in \mathbb{Z}_{>0}$. All the other cases are similar. Let $F_{1}$ be as in proposition 2.1 , so we have :

$$
\begin{aligned}
F_{1} & =\left\{\left(z_{1}, z_{2}\right) \in K^{2}|| \alpha_{1}|\leq| z_{1}|\leq 1,| z_{2}^{l}|\leq| z_{1}^{k} \mid\right\} \\
& =\left\{\left(z_{1}, z_{2}\right) \in Y|| \frac{\alpha_{1}}{z_{1}}|\leq 1,| \frac{z_{2}^{l}}{z_{1}^{k}} \mid \leq 1\right\} \\
& =\left\{\left(z_{1}, z_{2}\right) \in Y|| \frac{z_{2}^{l}}{z_{1}^{k}}|\leq 1,| \frac{\alpha_{1}^{s} z_{1}^{k-s}}{z_{1}^{k}} \mid \leq 1, s=1 . . k\right\} .
\end{aligned}
$$

It is clear that the set $\left\{z_{1}^{k}, \alpha_{1} z_{1}^{k-1}, \ldots, \alpha_{1}^{k}, z_{2}^{l}\right\}$ generates the unit ideal of $\left.K<z_{1}, z_{2}\right\rangle$, so $F_{1}$ is a monomial rational subspace of $Y$.

Remark. Let $v: K^{2} \rightarrow(\mathbb{R} \cup\{-\infty\})^{2}$ be the map defined by :

$$
\left(z_{1}, z_{2}\right) \rightarrow\left(\log \left|z_{1}\right|, \log \left|z_{2}\right|\right) \text {. }
$$

The image $v(Y)$ of $Y$ is given by :

$$
v(Y)=\left\{\left(x_{1}, x_{2}\right) \in(\mathbb{R} \cup\{-\infty\})^{2} \cap v\left(K^{2}\right) \mid x_{1} \leq 0, x_{2} \leq 0\right\} .
$$

The image of a monomial rational subspace of $Y$ is a convex domain in $v(Y)$ defined by a finite number $s$ of rational inequalities

$$
n_{i} x_{1}+m_{i} x_{2} \leq \log \left|c_{n_{i}, m_{i}}\right|, i=1 . . s
$$

coming from the monomial inequalities

$$
\left|\frac{f_{i}}{f_{0}}\right|=\left|\frac{z_{1}^{n_{i}} z_{2}^{m_{i}}}{c_{n_{i}, m_{i}}}\right| \leq 1, i=1 . . s, n_{i}, m_{i} \in \mathbb{Z}, c_{n_{i}, m_{i}} \in K^{*} .
$$


Proposition 2.2. A convex domain $C \subseteq v(Y)$, is the image $v(X)$ of a monomial rational subspace $X \subseteq Y$ if and only if $C$ satisfies the following two conditions $a$ and $b$ :

a) $C$ is defined by a finite number $s$ of rational inequalities :

$n_{i} x_{i}+m_{i} x_{2} \leq \log \left|c_{n_{i} m_{i}}\right|, n_{i}, m_{i} \in \mathbb{Z}, c_{n_{i} m_{i}} \in K^{*}, i=1 . . s$.

(When $K$ is contained in the algebraic closure of a local field then we can normalize the valuation on $K$ such that $\log \left|K^{*}\right| \subseteq \mathbb{Q}$. Then all the coefficients of these inequalities are really rational.)

b) $C$ has one of the following properties :

1) $\left\{\left(x_{1}, x_{2}\right) \in(\mathbb{R} \cup\{-\infty\})^{2} \cap v(Y) \mid x_{1}, x_{2} \leq a\right\} \subseteq C$ for some $a \in\left(\log \left|K^{*}\right|\right) \cap \mathbb{R}_{<0}$

2) $C \subseteq\left\{\left(x_{1}, x_{2}\right) \in(\mathbb{R} \cup\{-\infty\})^{2} \cap v(Y) \mid c \leq x_{1} \leq 0\right\}$ for some $c \in\left(\log \left|K^{*}\right|\right) \cap \mathbb{R}_{<0}$

3) $C \subseteq\left\{\left(x_{1}, x_{2}\right) \in(\mathbb{R} \cup\{-\infty\})^{2} \cap v(Y) \mid c \leq x_{2} \leq 0\right\}$ for some $c \in\left(\log \left|K^{*}\right|\right) \cap \mathbb{R}_{<0}$

4) $C \subseteq\left\{\left(x_{1}, x_{2}\right) \in(\mathbb{R} \cup\{-\infty\})^{2} \cap v(Y) \mid c \leq x_{1}, x_{2} \leq 0\right\}$ for some $c \in\left(\log \left|K^{*}\right|\right) \cap \mathbb{R}_{<0}$.

Proof. Let $X=\left\{z \in Y|| \frac{f_{i}(z)}{f_{0}(z)} \mid \leq 1, i=1 . . s\right\} \subseteq Y$ be a monomial rational subspace. In the last remark above we have already shown that the image $C=v(X) \subseteq v(Y)$ is given by a finite number of rational inequalities. So we only have to prove that $C=v(X)$ satisfies condition $b$.

Now $f_{0}(z)$ is one of the following monomials :
1) $f_{0}=c \quad, \quad c \in K^{*}$
2) $f_{0}=c z_{1}^{k} \quad, \quad c \in K^{*}, k \in \mathbb{Z}_{>0}$
3) $f_{0}=c z_{2}^{l} \quad, \quad c \in K^{*}, l \in \mathbb{Z}_{>0}$
4) $f_{0}=c z_{1}^{k} z_{2}^{l}, \quad c \in K^{*}, k, l \in \mathbb{Z}_{>0}$.

In case 1 we have $f_{0}=c \in K^{*}$, so all the monomials $\frac{f_{i}(z)}{f_{0}(z)}$ are monomials $c_{i} z_{1}^{n_{i}} z_{2}^{m_{i}}$ with $n_{i}, m_{i} \in \mathbb{Z}_{\geq 0}, c_{i} \in K^{*}$. This shows that $C=v(X)$ has property $b 1$. 
In the other cases we see that some $f_{i}=\alpha \in K^{*}$, since $f_{0}, \ldots f_{s}$ generate the unit ideal in $K\left\langle z_{1}, z_{2}\right\rangle$. This shows that in the cases 2,3 and 4 the convex domain $C=v(X)$ satisfies conditions $b 2, b 3$ and $b 4$ respectively.

We will only prove this explicitly in case 4. Now $z \in X$ satisfies $\left|\frac{\alpha}{z_{1}^{k} z_{2}^{T}}\right| \leq 1$ for some $k, l \in \mathbb{Z}_{>0}, \alpha \in K^{*}$. Therefore $v(X)$ satisfies the inequality : $-k x_{1}-l x_{2} \leq-\log |\alpha|$. Since $x_{1}, x_{2} \leq 0$ we have :

$$
\begin{gathered}
x_{1} \geq \frac{\log |\alpha|}{k}, x_{2} \geq \frac{\log |\alpha|}{l} \\
\Longrightarrow x_{1}, x_{2} \geq \min \left(\frac{\log |\alpha|}{k}, \frac{\log |\alpha|}{l}\right) \geq c \text { for some } c \in \log \left|K^{*}\right| \cap \mathbb{R}_{<0} .
\end{gathered}
$$$$
x_{1}=0
$$

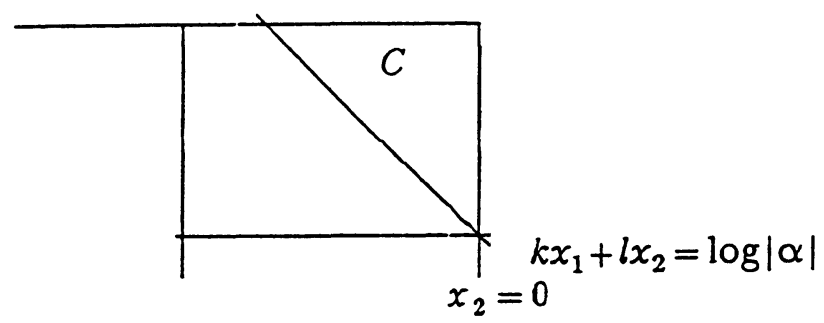

This shows that $C=v(X)$ satisfies condition $b 4$.

Now we will show that a convex domain $C$ that satisfies conditions $a$ and $b$ is the image $v(X)$ of a monomial rational subspace $X \subseteq Y$. Let $C$ be defined by the rational inequalities :

$$
n_{i} x_{1}+m_{i} x_{2} \leq \log \left|c_{n_{i}, m_{i}}\right|, n_{i}, m_{i} \in \mathbb{Z}, c_{n_{i}, m_{i}} \in K^{*}, i=1 . . s .
$$

Now $z \in v^{-1}(C)$ satisfies the inequalities :

$$
\left|\frac{z_{1}^{n_{i}} z_{2}^{m_{i}}}{c_{n_{i}, m_{i}}}\right| \leq 1, i=1 . . s
$$

Let $n, m$ be defined by

$$
n=\min \left(\left\{0, n_{1}, n_{2} \ldots n_{s}\right\}\right) \text { and } m=\min \left(\left\{0, m_{1}, m_{2} \ldots m_{s}\right\}\right) .
$$


Now we take $f_{0}=z_{1}^{n} z_{2}^{m}$ and define $f_{i} \in K<z_{1}, z_{2}>$ by

$$
\frac{f_{i}(z)}{f_{0}(z)}=\frac{z_{1}^{n_{i}} z_{2}^{m_{i}}}{c_{n_{i}, m_{i}}}, i=1 \cdots s .
$$

So we have $f_{i}(z)=\frac{z_{1}^{n_{i}+n} z_{2}^{m_{i}+m}}{c_{n_{i}, m_{i}}}, i=1 \cdots s$.

If $f_{0}(z)=1$ then $X=\left\{z \in Y|| \frac{f_{i}(z)}{f_{0}(z)} \mid \leq 1, i=1 . . s\right\}$ is a monomial rational subspace of $Y$ and $v^{-1}(C)=X$ and $C$ satisfies condition $a$ and $b 1$. If some $f_{i}(z) \in K^{*}$ for $s \geq i \geq 1$ then $v^{-1}(C)$ is again a monomial rational subspace of $Y$, since the $f_{i}$ generate the unit ideal in $K<z_{1}, z_{2}>$.

Now suppose $f_{0}=z_{1}^{n} z_{2}^{m}, n, m \in \mathbb{Z}_{>0}$ and $f_{i} \notin K, i=1$..s.

If $C$ satisfies condition $b 4$ we can find an element $c \in K^{*}$ such that $\left|\frac{c}{z_{1}^{n} z_{2}^{m}}\right| \leq 1$ for all $z \in v^{-1}(C)$. So taking $f_{s+1}=c$ we find a monomial rational subspace $X=v^{-1}(C)$ of $Y$ defined by $\left|\frac{f_{i}(z)}{f_{0}(z)}\right| \leq 1, i=1 \ldots s+1$.

If $C$ satisfies condition $b 2$ we can find a $c \in K^{*}$ such that $\left|\frac{c}{z_{1}}\right| \leq 1$ for all $z \in v^{-1}(C)$. Furthermore by the definition of $m$ there exists an $f_{i}(z)$ such that $\frac{f_{i}(z)}{f_{0}(z)}=\frac{z_{1}^{n_{i}}}{c_{n_{i}}, m z_{2}^{m}}, m>0$.

From this we see :

$$
\begin{aligned}
& \left|\frac{f_{i}(z)}{f_{0}(z)}\right|=\left|\frac{z_{1}^{n_{i}}}{c_{n_{i}, m} z_{2}^{m}}\right| \leq 1 \\
\Rightarrow & \begin{cases}\left|\frac{c}{z_{1}}\right|^{-n_{1}}\left|\frac{1}{z_{2}^{m}}\right| \leq\left|c_{n_{i}, m} c^{-n_{i}}\right| & \text { if } n_{i} \leq 1 \text { and }\left|\frac{c}{z_{i}}\right| \leq 1 \\
\left|z_{1}\right|^{n_{i}}\left|\frac{1}{z_{2}^{m}}\right| \leq\left|c_{n_{i}, m}\right| & \text { if } n_{i} \geq 0 \text { and }\left|z_{1}\right| \leq 1\end{cases} \\
\Rightarrow & \left|\frac{1}{z_{2}^{m}}\right| \leq|\alpha| \text { for some } \alpha \in K^{*} .
\end{aligned}
$$

So $C$ satisfies condition $b 4$, therefore we know $v^{-1}(C) \subseteq Y$ is a monomial rational subspace. If $C$ satisfies condition $b 3$ we again find that $C$ must satisfy condition $b 4$ if $f_{0}=z_{1}^{n} z_{2}^{m}, n, m \in \mathbb{Z}_{>0}$. If $f_{0}=z_{1}^{n} z_{2}^{m}, n, m>0$ then $C$ cannot satisfy condition $b 1$.

The situations with $f_{0}=z_{1}^{n}$ or $f_{0}=z_{2}^{m}$ are similar. 
Remark. Using proposition 2.2 above we can now describe explicitly the convex doamins $C \subseteq v(Y)$ such that $v^{-1}(C)=X$ is a monomial rational subspace $X \subseteq Y$.

In the next table we give description of $C$ and $X$ in the case int $(C)=\varnothing$.

\begin{tabular}{c|l}
$\mathrm{C}$ & $\mathrm{X}$ \\
\hline The empty set $\varnothing$ & $\left|c z_{1}\right| \geq 1$ and $|c|<1$ \\
a point $P=\left(\frac{1}{n} \log \left|c_{1}\right|, \frac{1}{m} \log \left|c_{2}\right|\right)$ & $\left|z_{1}^{n}\right|=\left|c_{1}\right|,\left|z_{2}^{m}\right|=\left|c_{2}\right| \leq 1, c_{1}, c_{2} \in K^{*}$ \\
a halfline $x_{1}=\frac{1}{n} \log \left|c_{1}\right|$ & $\left|z_{1}^{n}\right|=\left|c_{1}\right| \leq 1, \quad c_{1} \in K^{*}$ \\
$\begin{array}{c}\text { or } x_{2}=\frac{1}{m} \log \left|c_{2}\right| \\
\text { a line segment: }\end{array}$ & $\left|z_{2}^{m}\right|=\left|c_{2}\right| \leq 1, \quad c_{2} \in K^{*}$ \\
$\begin{array}{c}n x_{1}+m x_{2}=\log \left|c_{1}\right| \\
\text { and } \frac{1}{k} \log \left|c_{2}\right| \leq x_{1} \leq \frac{1}{l} \log \left|c_{3}\right| \\
\text { or } \frac{1}{k} \log \left|c_{4}\right| \leq x_{2} \leq \frac{1}{l} \log \left|c_{5}\right|\end{array}$ & $\left|z_{1}^{n} z_{2}^{m}\right|=\left|c_{1}\right| \leq 1, \quad c_{1} \in K^{*}$ \\
$\left|c_{2}\right| \leq\left|z_{1}\right|^{k},\left|z_{1}^{l}\right| \leq\left|c_{3}\right| \leq 1, c_{2}, c_{3} \in K^{*},\left|z_{2}^{l}\right| \leq\left|c_{5}\right| \leq 1, c_{4}, c_{5} \in K^{*}$
\end{tabular}

If $\operatorname{int}(C) \neq \varnothing$ then $C$ can have one of the following forms. The numbering corresponds with the one of property $b$ in proposition 2.2 .
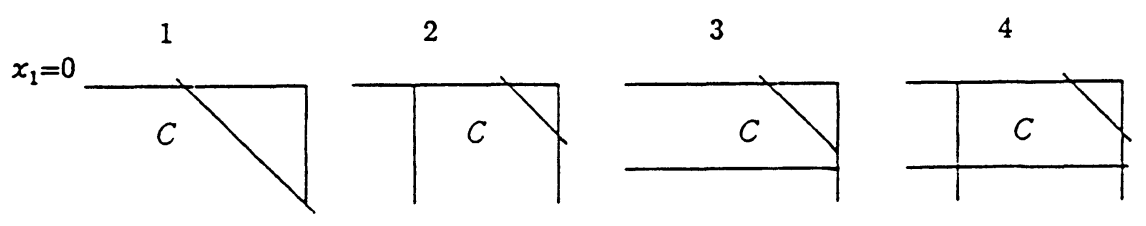

Definition. We define $\sqrt{\left|K^{*}\right|}$ as being the set

$$
\sqrt{\left|K^{*}\right|}=\left\{x \in \mathbb{R}\left|x^{n} \in\right| K^{*} \mid \text { for some } n \in \mathbb{Z}_{>0}\right\} \text {. }
$$

Let $C \subseteq v(Y)$ be a convex doamin. A point $P \in C \neq \varnothing$ is called an extremal point of $C$ if there exists no line segment $\left[P_{1}, P_{2}\right] \subseteq C$ with $P \neq P_{1}, P_{2}$ such that $P \in\left[P_{1}, P_{2}\right]$. 
Lemma 2.1. Let $\left|K^{*}\right|=\sqrt{\left|K^{*}\right|}$ and let $X \subseteq Y$ be a monomial rational domain such that $v(X)=C \neq \varnothing$ is a convex domain in $v(Y)$. Let $A$ be the affinoid algebra of $X$. For a polynomial $f \in K\left[z_{1}, z_{2}, z_{1}^{-1}, z_{2}^{-1}\right] \cap A$ we have $\|f\|=\left\|\sum a_{n, m} z_{1}^{n} z_{2}^{m}\right\|=\max \left|a_{n, m}\right|\left\|z_{1}^{n} z_{2}^{m}\right\|$ where \|\| denotes the spectral norm on the affinoid algebra $A$.

Let $P_{1} . . P_{k}$ be the extremal points of the convex domain $C$. Then we have :

$$
\left\|z_{1}^{n} z_{2}^{m}\right\|=\max \left\{\left|a^{n} b^{m}\right| \mid(a, b) \in \bigcup_{i=1}^{k} v^{-1}\left(P_{i}\right)\right\} .
$$

Proof. Since $\left|K^{*}\right|=\sqrt{\left|K^{*}\right|}$ and $K$ is non-archimedean we have $\|f\|=\max \left|a_{n, m}\right|\left\|z_{1}^{n} z_{2}^{m}\right\|$.

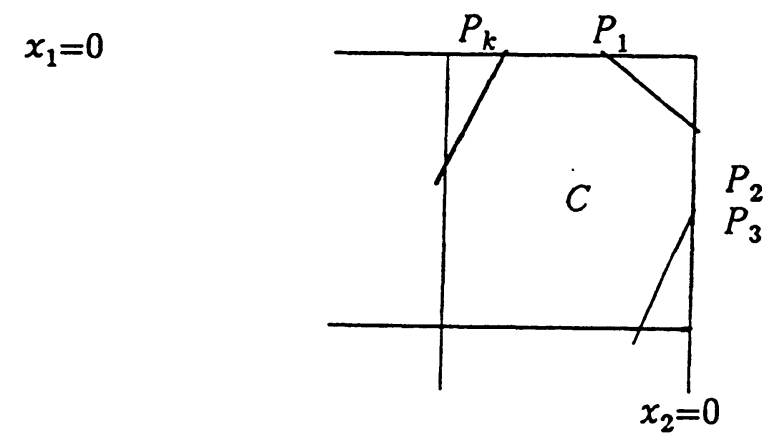

A monomial $z_{1}^{n} z_{2}^{m}$ has norm $|c|$ on the line $n x_{1}+m x_{2}=\log |c|$ in $v(Y)$. The maximal value $|c| \in\left|K^{*}\right|$ such that the line $n x_{1}+m x_{2}=\log |c|$ has at least one point in common with the convex domain $C$ is equal to $\left\|z_{1}^{n} z_{2}^{m}\right\|$. It is clear that this rational line can contain at most two points $P_{i}$. This only occurs when the monomial belongs to a rational line on the boundary of $C$. This shows that we have indeed : 


$$
\left\|z_{1}^{n} z_{2}^{m}\right\|=\max \left\{\left|a^{n} b^{m}\right| \mid(a, b) \in \bigcup_{i=1}^{k} v^{-1}\left(P_{i}\right)\right\}
$$

So for a polynomial $f=\sum a_{n, m} z_{1}^{n} z_{2}^{m} \in K\left[z_{1}, z_{2}, z_{1}^{-1}, z_{2}^{-1}\right] \cap A$ we have :

$$
\|f\|=\max _{n, m}\left|a_{n, m}\right| \max _{1 \leq i \leq k}\left|a_{i}^{n} b_{i}^{m}\right|
$$

Here $\left(a_{i}, b_{i}\right) \in v^{-1}\left(P_{i}\right)$ are chosen in $v^{-1}\left(P_{i}\right)$.

Definitions. Let $A$ be an affinoid algebra, with spectralnorm \|\| and let $X=S p(A)$. Let $K^{0}$ be the ring of integers of $K$, i.e.

$$
K^{0}:=\{x \in K|| x \mid \leq 1\}
$$

We define the $K^{0}$-module $A^{0}$ by $A^{0}:=\{f \in A \mid\|f\| \leq 1\}$. Now we define the $K^{0}$-submodule $A^{00} \subset A^{0}$ by $A^{00}:=\{f \in A \mid\|f\|<1\}$. We call $\bar{A}=A^{0} / A^{00}$ the reduction of $A$ and $\bar{X}=\operatorname{spec}(\bar{A})$ the reduction of $X$.

We have a map $R: X \rightarrow \bar{X}$. The image $R(m)$ of a maximal ideal $m$ of $A$ is a maximal ideal of $\bar{A}$ defined by :

$$
R(m)=\text { the image of } m \cap A^{0} i n \bar{A}=A^{0} / A^{00} .
$$

The map $R$ is surjective onto the set of closed points of $\bar{X}$ (see [BGR] p.270).

Remark. Let us take a monomial rational domain $X \subseteq Y, X \neq \varnothing$ such that $C=v(X)$ is a convex domain $\neq \varnothing$ in $v(Y)$. We can now associate to an extremal point $P_{i}$ of $C$ the monomials $z_{1}^{n} z_{2}^{m}, n, m \in \mathbb{Z}$ that are in the affinoid algebra $A$ of $X$ and attain their maximal value $\left\|z_{1}^{n} z_{2}^{m}\right\|$ in $v^{-1}\left(P_{i}\right)$. This gives a partition of the monomials in $A$.

Let $f$ map the monomials $z_{1}^{n} z_{2}^{m}$ into $\mathbb{Z}^{2}$ and be defined by :

$$
f\left(z_{1}^{n} z_{2}^{m}\right)=(n, m)
$$

Let $M_{i}$ be the set $M_{i}:=\left\{f\left(z_{1}^{n} z_{2}^{m}\right) \mid z_{1}^{n} z_{2}^{m}\right.$ is a monomial in $A$ and attains its maximal value $\left\|z_{1}^{n} z_{2}^{m}\right\|$ in $\left.v^{-1}\left(P_{i}\right)\right\}$. 
1

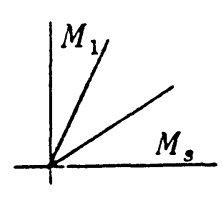

2

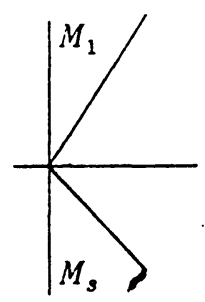

3

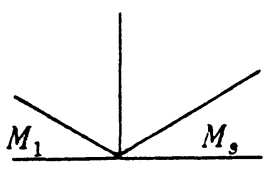

4

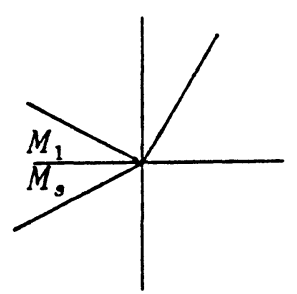

In the pictures above we have drawn the partitions. The figures $1,2,3$ and 4 correspond to monomial rational domains $X \subseteq Y$ that have property $b 1, b 2, b 3$ and $b 4$ respectively (see proposition 2.2 ).

The line between the areas $M_{i}$ and $M_{i+1}$ belongs to both, since it corresponds to the monomials that have their maximum value in both $v^{-1}\left(P_{i}\right)$ and $v^{-1}\left(P_{i+1}\right)$.

Lemma 2.2. Let $X \subseteq Y$ be a monomial rational domain with affinoid algebra $A$. Let $\left|K^{*}\right|=\sqrt{\left|K^{*}\right|}$. Then there is a 1 - 1 correspondance between the minimal prime ideals $p_{i}$ of $\bar{A}$ and the extremal points $P_{i}$ of $C=v(X)$. In fact we have :

$$
p_{i}=\left\{\bar{f} \in \bar{A}|| f\left(a_{i}, b_{i}\right) \mid<1,\left(a_{i}, b_{i}\right) \in v^{-1}\left(P_{i}\right)\right\}
$$

Proof. Since $\left|K^{*}\right|=\sqrt{\left|K^{*}\right|}$, we can choose for every monomial $z_{1}^{n} z_{2}^{m} \in$ $A$, a $c_{n, m} \in K^{*}$ such that $\left\|z_{1}^{n} z_{2}^{m}\right\|=c_{n, m}$. Now the $K^{\circ}$-module $A^{\circ}$ is generated by the elements $x_{n, m}:=\frac{z_{1}^{n} z_{2}^{m}}{c_{n, m}}$. So the $\bar{K}$-module $\bar{A}$ is generated by the images $\bar{x}_{n, m}$ of $x_{n, m}$ in $\bar{A}$. A straightforward calculation shows that

$$
\bar{x}_{n, m} \cdot \bar{x}_{k, l}=\delta \cdot \bar{x}_{n+k, m+l} \text { for some } \delta \in \bar{K} .
$$

Furthemore $\delta \in \bar{K}^{*}$ if and only if $\bar{x}_{n, m}$ and $\bar{x}_{k, l}$ are the images of monomials belonging to the same area $M_{i}$.

This shows that we have indeed for every extremal point $P_{i}$ of $C=v(X)$ a minimal prime ideal $p_{i}=\left\{\bar{f} \in \bar{A}|| f\left(a_{i}, b_{i}\right) \mid<1,\left(a_{i}, b_{i}\right) \in v^{-1}\left(P_{i}\right)\right\}$. The ideal $p_{i}$ is generated by the elements $\bar{x}_{n, m}$ with $(n, m) \notin M_{i}$, so $z_{1}^{n} z_{2}^{m}$ does not reach its maximum $\left\|z_{1}^{n} z_{2}^{m}\right\|$ in $v^{-1}\left(P_{i}\right)$. 
Remark. Let us look at $B_{i}=\bar{A} / p_{i}$. We see that this ring is generated over $\bar{K}$ by the elements $\bar{x}_{n, m},(n, m) \in M_{i} \cap \mathbb{Z}^{2}$. We can choose the constants $c_{n, m}$ such that the multiplication in $B_{i}$ is given by :

$$
\bar{x}_{n, m} \cdot \bar{x}_{k, l}=\bar{x}_{n+k, m+l},(n, m),(k, l) \in M_{i} \cap \mathbb{Z}^{2} .
$$

Since the areas $M_{i} \subseteq \mathbb{Z}^{2}$ are rational, the semigroup of points in $M_{i} \cap \mathbb{Z}^{2}$ is generated by a finite number of elements. This shows that $B_{i}$ is a finitely generated $\bar{K}$-algebra.

It is clear that the quotient field of $B_{i}$ is $\bar{K}\left(z_{1}, z_{2}\right)$.

Examples. We identify the monomials $\bar{x}_{n, m} \in B_{i}=\bar{A} / P_{i}$ with the points $(n, m) \in M_{i} \cap \mathbb{Z}^{2}$. For convenience we choose $B$ such that one of the borderlines goes through the point $(1,0)$. This can always be done by using a transformation by an element of $G L(2, \mathbb{Z})$.

Now the generators of $B$ over $\bar{K}$ are $\bar{x}_{1,0}$ and $\bar{x}_{m, 1}$.

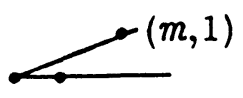

There are no relations between the generators, so we have :

$$
B=\bar{K}\left[\bar{x}_{1,0}, \bar{x}_{m, 1}\right] .
$$

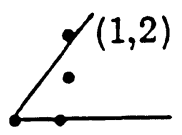

The generators of $B$ are $\bar{x}_{1,2}, \bar{x}_{1,1}$ and $\bar{x}_{1,0}$. We have the relations : $\bar{x}_{1,1}^{2}=\bar{x}_{1,2} \cdot \bar{x}_{1,0}$. So we have :

$$
B=\bar{K}\left[\bar{x}_{1,2}, \bar{x}_{1,1}, \bar{x}_{1,0}\right] /\left(\bar{x}_{1,1}^{2}-\bar{x}_{1,2} . \bar{x}_{1,0}\right) .
$$

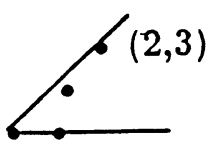

The generators of $B$ are $\bar{x}_{1,0}, \bar{x}_{1,1}$ and $\bar{x}_{2,3}$. We have the relations : $\bar{x}_{2,3} \bar{x}_{1,0}=\bar{x}_{1,1}^{3}$. So we have :

$$
B=\bar{K}\left[\bar{x}_{1,0}, \bar{x}_{1,1}, \bar{x}_{2,3}\right] /\left(\bar{x}_{1,1}^{3}-\bar{x}_{2,3} \cdot \bar{x}_{1,0}\right) .
$$

Remark. Our description of the algebra $B$ is in complete accordance with the theory of toroidal embeddings as described in [KKMS], [0.1] and [0.2]. We will now state and use some results and definitions from it.

Definitions. Let $M$ be the set of monomials $z_{1}^{n} z_{2}^{m}, n, m \in \mathbb{Z}$.

Now $M \simeq \mathbb{Z}^{2}$ where the isomorphism is given by the map

$$
f: z_{1}^{n} z_{2}^{m} \rightarrow(n, m) .
$$


Let $M_{\mathbb{R}}$ be $M_{\mathbb{R}}:=M \otimes \mathbb{R} \simeq \mathbb{R}^{2}$.

We call a semi-group $S \subseteq M$ saturated if $S$ satisfies :

$$
n r \in S \Rightarrow r \in S, \text { where } r \in M \text { and } n \in \mathbb{Z}_{>0}
$$

We call a convex domain in $M_{\mathbb{R}}$ bounded by two rational halflines starting in the origin a (convex rational polyhedral) cone. We will always assume that the cone does not contain a linear subspace. For a semi-group $S \subseteq M$ we define the space $X_{S}:=$ spec $\bar{K}\left[f^{-1}(S)\right]$. For a cone $\sigma \subset M_{\mathbb{R}}$, the set $\sigma \cap M$ is a saturated finitely generated semi-group. Moreover any finitely generated semi-group $S$, not containing a line, has the form $\sigma \cap M$ for some cone $\sigma$.

Theorem 2.1. Let $S \subset M$ be a semi-group that generates $M$ as a group. Let $\sigma \subset M_{\mathbb{R}}$ be a cone such that $\operatorname{int}(\sigma) \neq \varnothing$, so

$$
\sigma=\left\{\lambda\left(a e_{1}+b e_{2}\right)+\mu\left(c e_{1}+d e_{2}\right) \mid \lambda, \mu \in \mathbb{R}_{\geq 0}\right\}
$$

for some $\mathbb{Z}$-basis $\left\{e_{1}, e_{2}\right\}$ of $M$ and $a, b, c, d \in \mathbb{Z}$ with g.c.d. $(a, b)=$ g.c.d. $(c, d)=1$ and $n=\left|\operatorname{det}\left(\begin{array}{ll}a & b \\ c & d\end{array}\right)\right| \neq 0$. Now we have :

a) The space $X_{S}$ is normal if and only if $S$ is saturated.

b) The space $X_{\sigma}$ is non-singular if and only if the semi-group $\sigma \cap M$ is generated by a $\mathbb{Z}$-basis of $M$.

c) If $\bar{K}$ contains the $n$ - th roots of unity then $X_{\sigma} \cong \mathbb{A} \frac{2}{K} / \mu$, where $\mu$ is a cyclic group of order $n$ acting diagonally on $\mathbb{A} \frac{2}{K}$.

Proof. All this is proved in [KKMS] Ch.I $\S 1$. We shall recall the proof of part $c$ of the theorem, because this will give us a nice and explicit description of $X_{\sigma}$.

Let $\sigma$ be as in the theorem. We can choose a $\mathbb{Z}$-basis $\left\{f_{1}, f_{2}\right\}$ of $M$ such that $\sigma=\left\{\lambda f_{1}+\mu\left(k f_{1}+l f_{2}\right) \mid \lambda, \mu \in \mathbb{R}_{\geq 0}\right\} \subset M_{\mathbb{R}}$, where $k, l \in \mathbb{Z}$ with g.c.d. $(k, l)=1$. We may assume $l>0$, since we always can replace $f_{2}$ by $-f_{2}$. So we have $n=\left|\operatorname{det}\left(k f_{1}+l f_{2}, f_{1}\right)\right|=l$, since $\operatorname{det}\left(f_{1}, f_{2}\right)= \pm 1$.

If $n=l=1$ then $\left\{f_{1}, k f_{1}+l f_{2}\right\}$ is a $\mathbb{Z}$-basis of $M$. These two elements also generate $\sigma \cap M$. This makes it clear that :

$$
X_{\sigma}=\operatorname{spec} \bar{K}\left[f^{-1}\left(f_{1}\right), f^{-1}\left(k f_{1}+l f_{2}\right)\right] \cong \mathbb{A} \frac{2}{K}
$$


Let $l \neq 1$ and $l>0$. Now the semi-group $\sigma \cap M$ is not generated by $f_{1}$ and $k f_{1}+l f_{2}$. Let $M^{*}=\mathbb{Z} \cdot \frac{1}{l} f_{1} \oplus \mathbb{Z} \cdot f_{2}$. Now $\left\{\frac{1}{l} f_{1}, \frac{k}{l} f_{1}+f_{2}\right\}$ is a $\mathbb{Z}$-basis of $M^{*}$ and the two elements also generate the semi-group $\sigma \cap M^{*}$.

Let $g$ map the monomials $x^{u} y^{v}, u, v \in \mathbb{Z}$ into $M^{*}$ and be defined by

$$
g: x^{u} y^{v} \rightarrow u \cdot \frac{1}{l} f_{1}+v \cdot f_{2}
$$

It is clear that $\operatorname{spec} \bar{K}\left[g^{-1}\left(\sigma \cap M^{*}\right)\right] \cong \mathbb{A} \frac{2}{K}$ and $\sigma \cap M \subseteq \sigma \cap M^{*}$. Moreover we have $\operatorname{spec} \bar{K}\left[g^{-1}(\sigma \cap M)\right] \cong \operatorname{spec} \bar{K}\left[f^{-1}(\sigma \cap M)\right]$. This can be seen by using the map : $z_{1} \rightarrow x_{1}^{l}, z_{2} \rightarrow y$.

If $\bar{K}$ contains a primitive $l$-th root of unity $\zeta$ we can describe $X_{\sigma} \cong \operatorname{spec} \bar{K}\left[g^{-1}(\sigma \cap M)\right]$ as in the statement of the theorem. We can define an action $\tilde{\zeta}$ on $\mathbb{A} \frac{2}{K} \cong \operatorname{spec} \bar{K}\left[g^{-1}\left(\sigma \cap M^{*}\right)\right]$ by :

$$
\tilde{\zeta}(x)=\zeta \cdot x, \quad \tilde{\zeta}(y)=y
$$

The invariants of the group $\mu:=\langle\tilde{\zeta}>$ are generated by the monomials $x^{r l} y^{s}, r, s \in \mathbb{Z}$ that are in $g^{-1}\left(\sigma \cap M^{*}\right)$. So we have :

$$
\bar{K}\left[f^{-1}(\sigma \cap M)\right]=\bar{K}\left[g^{-1}(\sigma \cap M)\right]=K\left[g^{-1}\left(\sigma \cap M^{*}\right)\right]^{\mu} .
$$

This shows that : $X_{\sigma}=\mathbb{A} \frac{2}{K} / \mu$.

Lemma 2.3. Let $X \subseteq Y$ be a monomial rational domain such that $C=v(X)$ is a convex domain in $v(Y)$ with $\operatorname{int}(C) \neq \varnothing$. Let $P_{1}, . ., P_{S}$ be the extremal points of $C$. Let $M_{i}$ be the cone associated to the extremal point $P_{i}$ (See the remark just before lemma 2.2). Let $n_{i}=\left|\operatorname{det}\left(\begin{array}{ll}a & b \\ c & d\end{array}\right)\right|$, where $a x+b y=0$ and $c x+d y=0$ are the bordelines of $M_{i}$. Let $\bar{K}$ contain all the $n_{i}-t h$ roots of unity for $i=1 . . s$. Now the reduction $\bar{X}$ of the monomial rational domain $X$ is the following :

a) Every extremal point $P_{i}$ corresponds to exactly one affine surface $\mathbb{A} \frac{2}{K} / \mu_{i}$

b) If the line-segment $\left[P_{i} P_{j}\right]$ is part of the boundary of $C$, then the surfaces belonging to $P_{i}$ and $P_{j}$ have exactly one affine line $\mathbb{A} \frac{1}{K}$ in common.

c) If the line-segment $\left[P_{i} P_{j}\right]$ is not contained in the boundary of $C$, then the surfaces belonging to $P_{i}$ and $P_{j}$ have exactly one point in common. 
Proof. Let $A$ be the affinoid algebra of $X$.

In lemma 2.2 we proved that there is a $1-1$ correspondance between the points $P_{i}$ and the minimal prime ideals $p_{i}$ of $\bar{A}$. Now theorem 2.1 shows that $\operatorname{spec}\left(B_{i}\right):=\operatorname{spec}\left(\bar{A} / p_{i}\right)$ is the surface $\mathbb{A} \frac{2}{K} / \mu_{i}$ defined by the cone $M_{i}$.

Suppose the line-segment $\left[P_{i} P_{j}\right]$ is part of the boundary of $C$. The monomials in $\bar{A}$ that have an image $\neq 0$ in both $B_{i}$ and $B_{j}$ are the monomials $\left(\frac{z_{1}^{n} z_{2}^{m}}{c_{n, m}}\right)^{l}, l \in \mathbb{Z}_{\geq 0}$ which correspond to the rational line $P_{i} P_{j}$. This shows that the surfaces belonging to $P_{i}$ and $P_{j}$ have exactly one affine line $\mathbb{A} \frac{1}{K}$ in common.

Now suppose the line-segment $\left[P_{i} P_{j}\right]$ is not contained in the boundary of $C$. In this case there are no monomials in $\bar{A}$ which have a non-zero image in both $B_{i}$ and $B_{j}$. So the surfaces belonging to $P_{i}$ and $P_{j}$ can have at most one point in common. Of course they have the point defined by $\frac{z_{1}^{n} z_{2}^{m}}{c_{n, m}}=0$ for all $\frac{z_{1}^{n} z_{2}^{m}}{c_{n, m}}$ in $\bar{A}$ in common, since $\operatorname{int}(C) \neq \varnothing$.

Remark : We are looking for admissible affinoid coverings of $K^{2}-\{(0,0)\}$ that are invariant under the action of the group $\Gamma=\langle\gamma\rangle$. To find such coverings we use the fundamental domain of $\Gamma$ given in proposition 2.1.

First we need the notion of a pure covering, since we want the reductions of the affinoid space to glue together nicely.

Definition. Let $Z$ be a rigid analytic space.

A pure covering $\mathfrak{U}=\left(U_{i}\right)$ of $Z$ is an admissible covering by affinoid subspaces $U_{i}$ satisfying the following conditions :

1) For each $i, U_{i}$ intersects a finite number of $U_{j}$

2) If $U_{i} \cap U_{j} \neq \varnothing$ then there exists a Zariski-open affine set $V_{i j} \subset \bar{U}_{i}$ such that $U_{i} \cap U_{j}=R_{i}^{-1}\left(V_{i j}\right)$, where $R_{i}: U_{i} \rightarrow \bar{U}_{i}$ is the reduction, and $U_{i} \cap U_{j}$ is an affinoid space having reduction $R_{i j}: U_{i} \cap U_{j} \rightarrow V_{i j}$.

The word admissible in the definition means admissible with respect to a certain Grothendieck topology on $Z$.

Remark : There is a $1-1$ correspondence between pure coverings $\mathfrak{U}$ of a rigid analytic space $Z$ and formal schemes $\mathfrak{X}$ over $K^{0}$ such that the generic fibre of the map $\mathfrak{X} \rightarrow S p f K^{0}$ is the space $Z$. In this case the closed fibre of the map $\mathfrak{X} \rightarrow S p f K^{0}$ is the reduction of $Z$ with respect to the pure covering U. 
Indeed for an affinoid subspace $U_{i} \subset Z, U_{i} \in \mathfrak{U}$ with affinoid algebra $A_{i}=K\left\langle x_{1} \ldots x_{n}\right\rangle / I$ we have :

$$
A_{i}^{o}=\lim _{\longleftarrow} A_{i}^{o} / m^{s} A_{i}^{o}=\lim _{\longleftarrow}\left(K^{o}\left[x_{1} \ldots x_{n}\right] / I\right) / m^{s}\left(K^{o}\left[x_{1} \ldots x_{n}\right] / I\right) .
$$

Here is $m=K^{\circ 0}$ if the valuation is discrete, otherwise we take $m=(\pi)$ for some $0 \neq \pi \in K^{o o}$.

Now $S p f A_{i}^{o} \subset$ spec $K^{\circ}\left[x_{1} \ldots x_{n}\right] / I$ is the subspace defined by the ideal $m$. This shows that the map $S p f A_{i}^{o} \rightarrow S p f K^{o}$ has $S p\left(A_{i}\right)=U_{i}$ as its generic fibre and $\overline{S p\left(A_{i}\right)}=\bar{U}_{i}$ as its closed fibre. Now the properties 1 and 2 of the definition of a pure covering show that all maps $\operatorname{Spf} A_{i}^{o} \rightarrow \operatorname{Spf} K^{\circ}$ glue together nicely. So we get a formal scheme $\mathfrak{X} \rightarrow S p f K^{\circ}$ with $Z$ as its generic fibre and the reduction of $Z$ with respect to the pure covering $\mathfrak{U}$ as its closed fibre.

Lemma 2.4. Let $\left(X_{i}\right)$ be a covering of $K^{2}-\{(0,0)\}$, such that every $X_{i}$ is a monomial rational domain and $\operatorname{int}\left(C_{i}\right) \neq \varnothing$, where $C_{i}=v\left(X_{i}\right)$. Now $\left(C_{i}\right)$ is a covering of $v\left(K^{2}-\{(0,0)\}\right)$ by convex rational domains.

The covering $\left(X_{i}\right)$ of $K^{2}-\{(0,0)\}$ is pure if and only if :

1) For each $i, C_{i} \cap C_{j} \neq \varnothing$ for at most a finite number of $C_{j}$.

2) $\forall i, j, C_{i} \cap C_{j} \neq \varnothing \Leftrightarrow C_{i} \cap C_{j}$ is a point $P$ or

$$
\begin{array}{r}
C_{i} \cap C_{j}=C_{i} \cap L=C_{j} \cap L, \text { where } L \text { is a } \\
\text { rational line. }
\end{array}
$$

Proof. Let us first show that a covering as described in the statement of the lemma is pure. If $C_{i} \cap C_{j}=\varnothing$ then also $X_{i} \cap X_{j}=\varnothing$. Since $C_{i}$ intersects only a finite number of $C_{j}$, our covering satisfies condition 1 of the definition.

Let us assume $C_{i} \cap C_{j} \neq \varnothing$, so $C_{i} \cap C_{j}$ is a point $P$ or a rational line $L$ such that $C_{i} \cap L=C_{j} \cap L$. Now clearly $v^{-1}\left(C_{i} \cap C_{j}\right)$ is a affinoid subspace of $X_{i}=v^{-1}\left(C_{i}\right)$, since it is given by an equation $\left|x_{n, m}\right|=\left|\frac{z_{1}^{n} z_{2}^{m}}{c_{n, m}}\right|=1$ in $X_{i}$ if $C_{i} \cap C_{j}=L$, where $L$ is the rational line $n x_{1}+m x_{2}=\log \left|c_{n, m}\right|$. When $C_{i} \cap C_{j}$ is a point $P$ then $v^{-1}\left(C_{i} \cap C_{j}\right)$ is given by two such equations, coming from the two rational lines on the boundary of $C_{i}$ intersecting each other in the extremal point $P$. The situation in $C_{j}$ is identical.

In lemma 2.3 we proved that $\bar{X}_{i}$ is affine, so $v^{-1}\left(\overline{C_{i} \cap C_{j}}\right)$ is also affine in $\bar{X}_{i}$. The set $v^{-1}\left(\bar{C}_{i} \cap \overline{C_{j}}\right)$ is defined in $\bar{X}_{i}$ by one or two equations of the form $\bar{x}_{n, m} \neq 0$, so $v^{-1}\left(\overline{C_{i} \cap C_{j}}\right) \subset \bar{X}_{i}$ is open affine subset. 
Since $v^{-1}\left(C_{i} \cap C_{j}\right)$ describes the same affinoid subset of $K^{2}-\{(0,0)\}$ in both $X_{i}$ and $X_{j}$, we can identify $v^{-1}\left(\overline{C_{i} \cap C_{j}}\right)$ in both $\bar{X}_{i}$ and $\bar{X}_{j}$. This shows that our covering satisfies condition 2 of the definition, since it is clear that $v^{-1}\left(C_{i} \cap C_{j}\right)=R_{i}^{-1}\left(v^{-1}\left(\overline{C_{i} \cap C_{j}}\right)\right)$.

Let us now show that a covering $\left(X_{i}\right)$ of $K^{2}-\{(0,0)\}$ such that the covering $\left(C_{i}\right)$ of $v\left(K^{2}-\{(0,0)\}\right)$ does not satisfy condition 1 or 2 in the statement of the lemma is not pure. There are now three possibilities :

1) There exists an $i$ such that $C_{i} \cap C_{j} \neq \varnothing$ for an infinite number of $C_{j}$

2) There exists $i, j$ such that $C_{i} \cap C_{j}=C_{i} \cap C_{j} \cap L$, but $C_{i} \cap L \neq C_{j} \cap L, L$ is a rational line.

3) Int $\left(C_{i} \cap C_{j}\right) \neq \varnothing$ for some $i, j$.

It is easy to see that in all three cases the covering is not pure, since it does not satisfy some of the conditions in the definition above.

In case 1 it is clear that the covering $\left(X_{i}\right)$ does not satisfy condition 1 of the definition, since $X_{i}$ has a non-empty intersection with an infinite number of $C_{j}$.

In cases 2 and 3 the covering does not satisfy condition 2 of the definition above. In case 2 we have : $X_{i} \cap X_{j} \neq R_{i}^{-1}\left(V_{i j}\right)$ or $X_{i} \cap X_{j} \neq R_{j}^{-1}\left(V_{i j}\right)$. In case $3 v^{-1}\left(\overline{C_{i} \cap C_{j}}\right)$ is not open in $\bar{X}_{i}$ and $\bar{X}_{j}$.

Example : Let the group $\Gamma$ be generated by a contraction $\gamma$. In proposition 2.1 we constructed a fundamental domain $F$ for the action of $\Gamma$. The finite affinoid covering $\left(F_{i}\right)$ of $F$ we gave, where $F_{i}$ is a monomial rational domain, can be used to give a pure covering of $K^{2}-\{(0,0)\}$ that is $\Gamma$-invariant. Indeed the covering $\left(\gamma^{j}\left(F_{i}\right)\right)_{j \in Z}$ is $\Gamma$-invariant and pure by the lemma above if $\gamma$ has the form with $\lambda=0$. If $\lambda \neq 0$ then we can choose a small enough value of $|\lambda|$ such that $\gamma^{i}\left(F_{k}\right) \cap \gamma^{i+1}\left(F_{l}\right)$ and $\gamma^{i}\left(F_{k}\right) \cap \gamma^{i}\left(F_{l}\right), k, l=1,2$ are pure by the lemma above. Since $\gamma^{j}\left(F_{k}\right) \cap \gamma^{j}\left(F_{l}\right)=\varnothing$ if $|i-j| \geq 2$ the covering $\left(\gamma^{j}\left(F_{i}\right)\right)_{j \in \mathbb{Z}}$ is again pure.

We will now study in som detail the case where $\gamma$ is defined by :

$$
\gamma\left(z_{1}, z_{2}\right)=\left(\alpha_{1}\left(z_{1}+\lambda z_{2}^{m}\right), \alpha_{2} z_{2}\right), 0<\left|\alpha_{1}\right| \leq\left|\alpha_{2}\right|<1,|\lambda|<1
$$

and $\lambda=0$ if $\alpha_{1} \neq \alpha_{2}^{m}$ and satisfies the extra condition : $\left|\alpha_{1}^{k}\right|=\left|\alpha_{2}^{l}\right|$ for some $k, l \in \mathbb{Z}_{>0}$. 
In proposition 2.1. we showed that the fundamental domain $F$ can be covered by the following two affinoid subspaces :

$$
F_{1}=S p K<z_{1}, \frac{\alpha_{1}}{z_{1}}, \frac{z_{2}^{l}}{z_{1}^{k}}>\text { and } F_{2}=S p K<z_{2}, \frac{\alpha_{2}}{z_{2}}, \frac{z_{1}^{k}}{z_{2}^{l}}>
$$
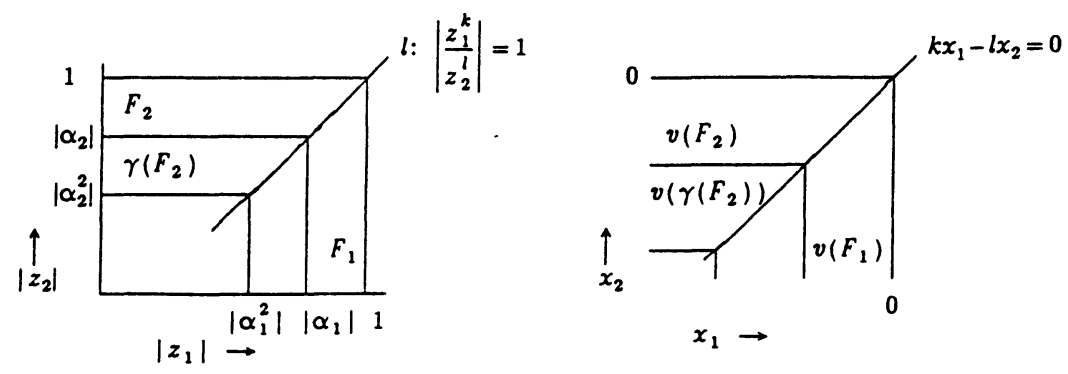

In the next lemma we will study the special case $\left|\alpha_{1}\right|=\left|\alpha_{2}\right|$. Then we will study the case $\left|\alpha_{1}^{k}\right|=\left|\alpha_{2}^{l}\right|$.

LEMMA 2.5. Let $\Gamma$ be generated by a contraction $\gamma$ such that $\left|\alpha_{1}\right|=\left|\alpha_{2}\right|$. Let $\left\{\gamma^{i}\left(F_{1}\right), \gamma^{j}\left(F_{2}\right) \mid i, j \in \mathbb{Z}\right\}$ be the pure $\Gamma$-invariant covering $C$ of $K^{2}-\{(0,0)\}$ given above. The reduction of $K^{2}-\{(0,0)\}$ with respect to this covering $C$ has for every extremal point $P$ of the convex domains $v\left(\gamma^{i}\left(F_{j}\right)\right), j=1,2, i \in \mathbb{Z}$, a surface $\tilde{\mathbb{P}}_{\bar{K}}^{2}$, i.e. a $\mathbb{P}_{\bar{K}}^{2}$ blown up in one point. The surface $\tilde{\mathbb{P}}_{\bar{K}}^{2}$ corresponding to the extremal points $P$ and $\gamma(P)$ have one $\mathbb{P} \frac{1}{K}$ in common, this line is exceptional in the $\tilde{\mathbb{P}}_{K}^{2}$ belonging to $P$ and ordinary in the other.

Proof. Let $\gamma^{i}\left(A_{j}\right), j=1,2, i \in \mathbb{Z}$ be the affinoid algebra belonging to $\gamma^{i}\left(F_{j}\right)$. Let $\left[\gamma^{i}\left(A_{j}\right)\right]_{P}$ be the component of the reduction of $\gamma^{i}\left(A_{j}\right)$ that correponds to the extremal point $P$ of the convex domain

$$
v\left(\gamma^{i}\left(A_{j}\right)\right) \subset v\left(K^{2}-\{(0,0)\}\right) .
$$

Since the covering $C$ is $\Gamma$-invariant and $\Gamma$ acts transitively on the sets of extremal points, it is sufficient to look at one extremal point $P$. We choose $P=\left(\log \left|\alpha_{1}\right|, \log \left|\alpha_{2}\right|\right)$. The point $P$ is an extremal point of the following four convex domains in $v\left(K^{2}-\{(0,0)\}\right): v\left(F_{1}\right), v\left(F_{2}\right), v\left(\gamma\left(F_{1}\right)\right)$ and $v\left(\gamma\left(F_{2}\right)\right)$. So we have to consider the following affinoid algebras and their 
reduction in $P$ :

$$
\begin{aligned}
A_{1} & =K<z_{1}, \frac{\alpha}{z_{1}}, \frac{z_{2}}{z_{1}}> & {\left[\bar{A}_{1}\right]_{P} } & =\bar{K}\left[\frac{\bar{\alpha}}{z_{1}}, \frac{\bar{z}_{2}}{z_{1}}\right] \\
\gamma\left(A_{1}\right) & =K<\frac{z_{1}}{\alpha}, \frac{\alpha^{2}}{z_{1}}, \frac{z_{2}}{z_{1}}> & {\left[\gamma \overline{\left(A_{1}\right)}\right]_{P} } & =\bar{K}\left[\frac{\bar{z}_{1}}{\alpha}, \frac{\bar{z}_{2}}{z_{1}}\right] \\
A_{2} & =K<z_{2}, \frac{\alpha}{z_{2}}, \frac{z_{1}}{z_{2}}> & {\left[\bar{A}_{2}\right]_{P} } & =\bar{K}\left[\frac{\bar{\alpha}}{z_{2}}, \frac{\bar{z}_{1}}{z_{2}}\right] \\
\gamma\left(A_{2}\right) & =K<\frac{z_{2}}{\alpha}, \frac{\alpha^{2}}{z_{2}}, \frac{z_{1}}{z_{2}}> & {\left[\gamma \overline{\left(A_{2}\right)}\right]_{P} } & =\bar{K}\left[\frac{\bar{z}_{2}}{\alpha}, \frac{\bar{z}_{1}}{z_{2}}\right]
\end{aligned}
$$

Here $\alpha \in K$ is chosen such that $|\alpha|=\left|\alpha_{1}\right|=\left|\alpha_{2}\right|$. We will now glue the reductions together.

The glueing of $\left[\bar{A}_{1}\right]_{P}$ and $\left[\overline{\gamma\left(A_{1}\right)}\right]_{P}$ along their intersection defined by $\frac{\bar{\alpha}}{z_{1}} \neq 0, \frac{\bar{z}_{1}}{\alpha} \neq 0$ and identifying $\left(\frac{\bar{\alpha}}{z_{1}}\right)^{-1}$ with $\frac{\overline{z_{1}}}{\alpha_{1}}$ gives us a $\mathbb{P} \frac{1}{K} \times \mathbb{A} \frac{1}{K}$ and has coordinate ring $\bar{K}\left[x_{1}, x_{3}\right] \times \bar{K}\left[\frac{y_{0}}{y_{1}}\right]$ where $\frac{x_{1}}{x_{3}}=\frac{\bar{z}_{1}}{\alpha}, \frac{x_{3}}{x_{1}}=\frac{\bar{\alpha}}{z_{1}}$ and $\frac{y_{0}}{y_{1}}=\frac{\bar{z}_{2}}{z_{1}}$.

The glueing of $\left[\bar{A}_{2}\right]_{P}$ and $\left[\overline{\gamma\left(A_{2}\right)}\right]_{P}$ gives us again a $\mathbb{P} \frac{1}{K} \times \mathbb{A} \frac{1}{K}$ defined by $\bar{K}\left[x_{0}, x_{2}\right] \times \bar{K}\left[\frac{y_{1}}{y_{0}}\right]$, where $\frac{x_{2}}{x_{0}}=\frac{\bar{\alpha}}{z_{2}}, \frac{x_{0}}{x_{2}}=\frac{\bar{z}_{2}}{\alpha}$ and $\frac{y_{1}}{y_{0}}=\frac{\bar{z}_{1}}{z_{2}}$.

Now we have to glue these two surfaces $\mathbb{P} \frac{1}{K} \times \mathbb{A} \frac{1}{K}$ along their intersection given by $\frac{y_{0}}{y_{1}} \neq 0, \frac{y_{1}}{y_{0}} \neq 0$. So we identify $\left(\frac{y_{0}}{y_{1}}\right)^{-1}$ with $\frac{y_{1}}{y_{0}}$ and use the relations :

$$
\frac{x_{1}}{x_{3}} \cdot \frac{x_{2}}{x_{0}}=\frac{\bar{z}_{1}}{\alpha} \cdot \frac{\bar{\alpha}}{z_{2}}=\frac{\bar{z}_{1}}{z_{2}}=\frac{y_{0}}{y_{1}} \quad \text { and } \quad \frac{x_{3}}{x_{1}} \cdot \frac{x_{0}}{x_{2}}=\frac{y_{1}}{y_{0}} .
$$

So we have to identify $x_{0}$ with $x_{1}$, or $x_{3}$ with $x_{2}$. We choose $x_{2}=x_{3}$. Now the glueing gives us a homogeneous coordinate ring

$$
\bar{K}\left[x_{0}, x_{1}, x_{2}\right] \times \bar{K}\left[y_{0}, y_{1}\right] /\left(x_{0} y_{0}-x_{1} y_{1}\right) .
$$

This is the coordinate ring of a surface $\tilde{\mathbb{P}}_{\bar{K}}^{2}($ see $[\mathrm{H}])$. We have

$$
\frac{x_{0}}{x_{2}}=\frac{\overline{z_{2}}}{\alpha}, \frac{x_{1}}{x_{2}}=\frac{\overline{z_{1}}}{\alpha}, \frac{y_{0}}{y_{1}}=\frac{\overline{z_{2}}}{z_{1}}, \text { etc... }
$$




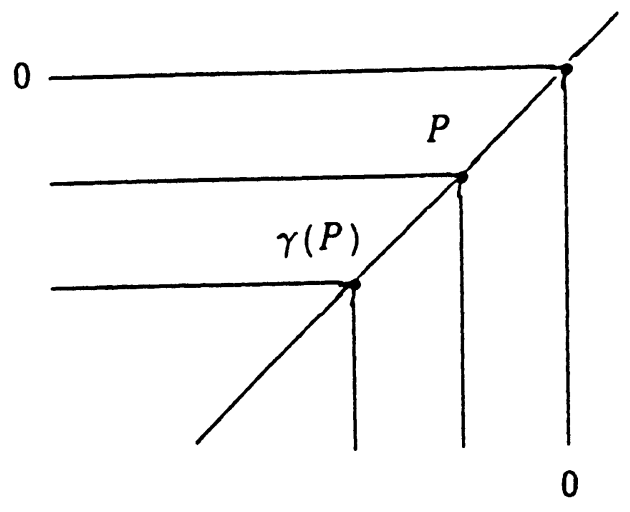

In the point $\gamma(P)$ we also find a surface $\widetilde{\mathbb{P}}_{\bar{K}}^{2}$ given by the homogeneous coordinate ring $\bar{K}\left[\frac{x_{0}}{\alpha}, \frac{x_{1}}{\alpha}, x_{2}\right] \times \bar{K}\left[\frac{y_{0}}{\alpha}, \frac{y_{1}}{\alpha}\right] /\left(\frac{x_{0}}{\alpha} \frac{y_{0}}{\alpha}-\frac{x_{1} y_{1}}{\alpha \alpha}\right)$. We will now describe the intersection of these two surfaces $\widetilde{\mathbb{P}}_{\bar{K}}^{2}$. In the $\widetilde{\mathbb{P}}_{\bar{K}}^{2}$ belonging to $P$ this intersection is determined by $\left|\frac{z_{1}}{\alpha}\right|,\left|\frac{z_{2}}{\alpha}\right|<1$. In the coordinate ring this space is determined by $x_{0}=x_{1}=0$. So we find the exceptional line given by $\bar{K}[0,0,1] \times \bar{K}\left[y_{0}, y_{1}\right]$ in the $\widetilde{\mathbb{P}}_{\bar{K}}^{2}$ belonging to $P$.

In the $\widetilde{\mathbb{P}}_{\bar{K}}^{2}$ belonging to $\gamma(P)$ the intersection is defined by $\left|\frac{\alpha^{2}}{z_{1}}\right|,\left|\frac{\alpha^{2}}{z_{2}}\right|<1$. So in the coordinate ring this space is given by

$$
x_{2} /\left(\frac{x_{0}}{\alpha}\right)=x_{2} /\left(\frac{x_{1}}{\alpha}\right)=0,
$$

therefore $x_{2}=0$. We find the ordinary line given by

$$
\bar{K}\left[\frac{x_{0}}{\alpha}, \frac{x_{1}}{\alpha}, 0\right] \times \bar{K}\left[\frac{y_{0}}{\alpha}, \frac{y_{1}}{\alpha}\right] /\left(\frac{x_{0} y_{0}}{\alpha \alpha}-\frac{x_{1} y_{1}}{\alpha \alpha}\right)
$$

in the $\widetilde{\mathbb{P}}_{\bar{K}}^{2}$ belonging to $\gamma(P)$.

So the reduction of $K^{2}-\{(0,0)\}$ with respect to the covering $\mathcal{C}$ is given by a string of surfaces $\widetilde{\mathbb{P}}_{K}^{2}$, glued together as in the figure by identifying an exceptional line $e$ in one $\widetilde{\mathbb{P}}_{\bar{K}}^{2}$ with an ordinary line $o$ in the text surface $\widetilde{\mathbb{P}}_{\bar{K}}^{2}$.

$$
\stackrel{o}{-\widetilde{\mathbb{P}}_{\bar{K}}^{2}} \frac{e \quad o}{\widetilde{\mathbb{P}}_{\bar{K}}^{2}} \frac{e \quad o}{\widetilde{\mathbb{P}}_{\bar{K}}^{2}} \frac{e \quad o}{\tilde{\mathbb{P}}_{\bar{K}}^{2}} \frac{e \quad o}{\widetilde{\mathbb{P}}_{\bar{K}}^{2}} \frac{e}{}
$$


Remark : Since the group $\Gamma$ above acts transitively on the set of extremal points, the reduction of the Hopf surface $K^{2}-\{(0,0)\} / \Gamma$ is given by a surface $\widetilde{\mathbb{P}}_{\bar{K}}^{2} / \sim$, where $\sim$ is the relation that identifies the exceptional line $e$ with the ordinary line $o$, when we use the covering $\mathcal{C}$ of $K^{2}-\{(0,0)\}$ given above.

Theorem 2.2. Let $\Gamma=\langle\gamma\rangle$ be generated by a contraction $\gamma$ with $\left|\alpha_{1}^{k}\right|=\left|\alpha_{2}^{l}\right|$ for some $k, l \in \mathbb{Z}_{>0}$ with g.c.d. $(k, l)=1$ and suppose $K$ contains a primitive $k \cdot l$-th root of unity. Let $F$ be the fundamental domain of $\Gamma$ constructed in proposition 2.1. Let $\mathcal{C}$ be the pure $\Gamma$-invariant covering $\left\{\gamma^{i}\left(F_{1}\right), \gamma^{j}\left(F_{2}\right) \mid i, j \in \mathbb{Z}\right\}$ of $K^{2}-\{(0,0)\}$.

Every extremal point $P$ of a convex domain $v\left(\gamma^{i}\left(F_{j}\right)\right), j=1,2, i \in \mathbb{Z}$ corresponds to a surface $\widetilde{\mathbb{P}}_{\bar{K}}^{2} / \mu_{k, l}$ in the reduction. The group $\mu_{k, l}$ is a finite cyclic group of order $k l$ acting diagonally on $\widetilde{\mathbb{P}}_{\bar{K}}^{2}$. The surfaces $\widetilde{\mathbb{P}}_{\bar{K}}^{2} / \mu_{k, l}$ belonging to the extremal points $P$ and $\gamma(P)$ have a line in common. This line is the image of the exceptional line in the $\widetilde{\mathbb{P}}_{\bar{K}}^{2} / \mu_{k, l}$ belonging to $P$ and it is the image of an ordinary line in the surface belonging to the extremal point $\gamma(P)$.

Proof. We only have to prove the theorem for one extremal point $P$, since $\Gamma$ acts transitively on the set of extremal points. We choose $P=\left(\log \left|\alpha_{1}\right|, \log \left|\alpha_{2}\right|\right)$. So $P$ is an extremal point of the following four convex domains in $v\left(K^{2}-\{(0,0)\}\right)$ :

$$
v\left(F_{1}\right), v\left(F_{2}\right), v\left(\gamma\left(F_{1}\right)\right) \text { and } v\left(\gamma\left(F_{2}\right)\right) .
$$

The associated affinoid algebras and their reductions in $P$ are :

$$
\begin{array}{rlrl}
A_{1} & =K<z_{1}, \frac{\alpha_{1}}{z_{1}}, \frac{z_{2}^{l}}{z_{1}^{k}}> & {\left[\overline{A_{1}}\right]_{P}} & =\bar{K}\left[\frac{\overline{\alpha_{1}}}{z_{1}}, \cdots, \frac{\bar{z}_{2}^{l}}{z_{1}^{k}}\right] \\
\gamma\left(A_{1}\right) & =K<\frac{z_{1}}{\alpha_{1}}, \frac{\alpha_{1}^{2}}{z_{1}} \frac{z_{2}^{l}}{z_{1}^{k}}> & {\left[\overline{\gamma\left(A_{1}\right)}\right]_{P}} & =\bar{K}\left[\frac{\overline{z_{1}}}{\alpha_{1}}, \cdots, \frac{\bar{z}_{2}^{l}}{z_{1}^{k}}\right] \\
A_{2} & =K<z_{2}, \frac{\alpha_{2}}{z_{2}}, \frac{z_{1}^{k}}{z_{2}^{l}}> & {\left[\overline{A_{2}}\right]_{P}=\bar{K}\left[\frac{\alpha_{2}}{z_{2}}, \cdots, \frac{\bar{z}_{1}^{k}}{z_{2}^{l}}\right]} \\
\gamma\left(A_{2}\right) & \left.=K<\frac{z_{2}}{\alpha_{2}}, \frac{\alpha_{2}^{2}}{z_{2}}, \frac{z_{1}^{k}}{z_{2}^{l}}\right\rangle & {\left[\overline{\gamma\left(A_{2}\right)}\right]_{P}} & =\bar{K}\left[\frac{\overline{z_{2}}}{\alpha_{2}}, \cdots, \frac{\bar{z}_{1}^{k}}{z_{2}^{l}}\right]
\end{array}
$$


Since $\operatorname{char}(K) \nmid k, l$, we can use theorem 2.1.c to get a more convenient description of $\left[\bar{A}_{i}\right]_{P}, i=1,2$ and $\left[\overline{\gamma\left(A_{i}\right)}\right]_{P}, i=1,2$. Let us take new variables $u, v$ such that $u^{l}=\frac{z_{1}}{\alpha_{1}}$ and $v^{l}=\frac{z_{2}}{\alpha_{2}}$. Now we have :

$$
\begin{aligned}
{\left[\overline{A_{1}}\right]_{P} } & =\bar{K}\left[u^{-1}, \frac{\overline{z_{2}}}{\alpha_{2}} / u^{k}\right]^{\mu}, \mu=<\widetilde{\zeta}> \\
{\left[\gamma\left(\overline{A_{1}}\right)\right]_{P} } & =\bar{K}\left[u, \frac{\overline{z_{2}}}{\alpha_{2}} / u^{k}\right]^{\mu} \\
{\left[\overline{A_{2}}\right]_{P} } & =\bar{K}\left[v^{-1}, \frac{\overline{z_{1}}}{\overline{\alpha_{1}}} / v^{l}\right]^{\mu} \\
{\left[\gamma\left(\overline{A_{2}}\right)\right]_{P} } & =\bar{K}\left[v, \frac{\overline{z_{1}}}{\alpha_{1}} / v^{l}\right]^{\mu}
\end{aligned}
$$

Here $\widetilde{\zeta}$ is defined by $\widetilde{\zeta}(u)=\zeta^{k} \cdot u, \widetilde{\zeta}(v)=\zeta^{l} \cdot v, \widetilde{\zeta}\left(\frac{\overline{z_{2}}}{\alpha_{2}}\right)=\frac{\overline{z_{2}}}{\alpha_{2}}, \widetilde{\zeta}\left(\frac{\overline{z_{1}}}{\alpha_{1}}\right)=\frac{\overline{z_{1}}}{\alpha_{1}}$, where $\zeta$ is a primitive $k \cdot l-t h$ root of unity.

Furthermore we have :

$$
\begin{aligned}
& \left(u^{-1}\right)^{r}\left(\frac{v}{u}\right)^{s} \in \bar{K}\left[u^{-1}, v / u\right]^{\mu} \\
\Leftrightarrow & k l \mid-k r+(l-k) s \\
\Leftrightarrow & k|s \wedge l| r+s, \text { since } g . c . d . \quad(k, l)=1 \\
\Leftrightarrow & \left(u^{-1}\right)^{r}\left(\frac{v}{u}\right)^{s} \in \bar{K}\left[u^{-1}, \frac{v^{k}}{u^{k}}\right]^{\mu}=\bar{K}\left[u^{-1}, \frac{\overline{z_{2}}}{\alpha_{2}} / u^{k}\right]^{\mu}=\left[\overline{A_{1}}\right]_{P} .
\end{aligned}
$$

This shows that we have in fact :

$$
\begin{aligned}
& {\left[\bar{A}_{1}\right]_{P}=\bar{K}\left[u^{-1}, \frac{\overline{z_{2}}}{\alpha_{2}} / u^{k}\right]^{\mu}=\bar{K}\left[u^{-1}, \frac{v^{k}}{u^{k}}\right]^{\mu}=\bar{K}\left[u^{-1}, \frac{v}{u}\right]^{\mu}} \\
& {\left[\gamma\left(\bar{A}_{1}\right)\right]_{P}=\bar{K}\left[u, \frac{\overline{z_{2}}}{\alpha_{2}} / u^{k}\right]^{\mu}=\bar{K}\left[u, \frac{v^{k}}{u^{k}}\right]^{\mu}=\bar{K}\left[u, \frac{v}{u}\right]^{\mu}} \\
& {\left[\bar{A}_{2}\right]_{P}=\bar{K}\left[v^{-1}, \frac{\overline{z_{1}}}{\alpha_{1}} / v^{l}\right]^{\mu}=\bar{K}\left[v^{-1}, \frac{u^{l}}{v^{l}}\right]^{\mu}=\bar{K}\left[v^{-1}, \frac{u}{v}\right]^{\mu}} \\
& {\left[\gamma\left(\bar{A}_{2}\right)\right]_{P}=\bar{K}\left[v, \frac{z_{1}}{\alpha_{1}} / v^{l}\right]^{\mu}=\bar{K}\left[v, \frac{u^{l}}{v^{l}}\right]^{\mu}=\bar{K}\left[v, \frac{u}{v}\right]^{\mu}}
\end{aligned}
$$

The group $\mu=\mu_{k, l}$ works identical on the intersections of the affine spaces. So we can interchange the group action and the glueing. 
In lemma 2.5 we already proved that the glueing gives us a surface $\widetilde{\mathbb{P}}_{\bar{K}}^{2}$. The group $\mu$ acts diagonally on this surface $\widetilde{\mathbb{P}}_{\bar{K}}^{2}$.

Let $K\left[x_{0}, x_{1}, x_{2}\right] \times K\left[y_{0}, y_{1}\right] /\left(x_{0} y_{0}-x_{1} y_{1}\right)$ be the homogeneous coordinate ring of $\widetilde{\mathbb{P}}_{\bar{K}}^{2}$. Here $\frac{x_{0}}{x_{2}}=u, \frac{x_{1}}{x_{2}}=v, \frac{y_{0}}{y_{1}}=\frac{u}{v}$ etc. This shows that the action of $\mu$ is defined by :

$$
\begin{aligned}
& \widetilde{\zeta}\left(x_{0}\right)=\zeta^{k} x_{0} \\
& \widetilde{\zeta}\left(x_{1}\right)=\zeta^{l} x_{1} \\
& \widetilde{\zeta}\left(x_{2}\right)=x_{2} \\
& \widetilde{\zeta}\left(y_{0}\right)=\zeta^{l} y_{0} \\
& \widetilde{\zeta}\left(y_{1}\right)=\zeta^{k} y_{1}
\end{aligned}
$$$$
\zeta^{k l}=1, \mu=\langle\widetilde{\zeta}>.
$$

Since the finite group $\mu$ acts diagonally, the invariants of $\mu$ are generated by monomials. If we forget about degree the generators are :

$$
x_{2}, x_{0}^{i} y_{1}^{l-i}, i=0 \cdots l, x_{1}^{i} y_{0}^{k-i}, i=0 \cdots k .
$$

The homogeneous algebra of invariants is generated by the monomials of degree $k l$ in the $x_{i}$ and/or $y_{i}$ that are $\mu$-invariant. So the generators are :

$$
\begin{array}{ll}
x_{2}^{k l-i-j} x_{0}^{i} x_{1}^{j} y_{0}^{r k-j} y_{1}^{s l-i} & r l+s k-i-j=k l \\
y_{0}^{k l}, y_{1}^{k l} & \\
x_{2}^{k l-i k-j l} x_{0}^{j l} x_{1}^{i k} & i k+j l \leq k l .
\end{array}
$$

Of course there are relations between these generators. One directly sees that there are relations of the form $s_{1} s_{2}=s_{3} s_{4}$, where the $s_{i}$ are some generators such that $s_{1} s_{2}$ is the same monomial as $s_{3} s_{4}$.

The relation $x_{0} y_{0}-x_{1} y_{1}=0$ gives rise to the following set of relations :

$$
x_{2}^{k l-i-j} x_{0}^{i} x_{1}^{j} y_{0}^{a l-j} y_{1}^{b k-i}-x_{2}^{k l-r-s} x_{1}^{r} x_{0}^{s} y_{0}^{a l-r} y_{1}^{b k-s}=0,
$$

where $i+j=r+s$ and $a l+b k-i-j=k l$.

This shows that we can reduce the number of generators. We will not go into this any further.

Since the surfaces $\widetilde{\mathbb{P}} \frac{2}{K} / \mu_{k, l}$, belonging to the extremal points $P$ and $\gamma(P)$, are the images of surfaces $\widetilde{\mathbb{P}}_{K}^{2}$, belonging to $P$ and $\gamma(P)$, which intersect each other as in lemma 2.5, the last part of the theorem is clear. 
Remark : Let $\mathcal{C}=\left(X_{i}\right)$ be a pure affinoid covering of $K^{2}-\{(0,0)\}$ such that all $X_{i}$ are monomial rational domains. Every extremal point $P$ of a convex rational domain $\mathcal{C}_{i}=v\left(X_{i}\right)$ gives a surface in the reduction of $K^{2}-\{(0,0)\}$ with respect to $\mathcal{C}$. The surface belonging to $P$ is the surface given by a conic decomposition of $\mathbb{R}^{2}$. Every cone $\sigma$ gives a surface $X_{\vee}$, where $\stackrel{v}{\sigma}$ is the dual cone of $\sigma$. So $\stackrel{\vee}{\sigma}$ is given by the points $(n, m) \in \mathbb{R}^{2}$ such that the lines $n x+m y$ have only values $\geq 0$ on $\sigma$. The surface $X_{v}$ is the component of the reduction of $X_{i}$ in $P$ and $\sigma$ is the cone given by the two half-lines through $P$ bounding $C_{i}=v\left(X_{i}\right)$. We have $X_{\vee}=\operatorname{spec} K\left[f^{-1}\left(\stackrel{\vee}{\sigma} \cap \mathbb{Z}^{2}\right)\right]$, where $f$ is the map $f: z_{1}^{n} z_{2}^{m} \rightarrow(n, m)$. In fact we have $\stackrel{\vee}{\sigma}=f\left(\bar{B}_{i}\right)$, where $B_{i}$ is the set of the monomials in the affinoid algebra $A_{i}$ of $X_{i}$ that obtain their maximum value in $P$. The glueing of the surfaces $X_{\sigma}$ for the cones $\sigma$ defined by $P$ gives us the surface in the reduction belonging to $P$.

We will now study coverings $\mathcal{C}$ such that to every extremal point $P$ belongs a non-singular surface.

Definitions. A conic decomposition is called regular if the surface $X_{\checkmark}$ is non-singular for every cone $\sigma$ in the decomposition.

A regular conic decomposition is called minimal if there are no cones $\sigma_{i}$ and $\sigma_{j}$ in the decomposition such that the decomposition obtained by replacing $\sigma_{i}$ and $\sigma_{j}$ by their union $\sigma_{i} \cup \sigma_{j}$ is again a regular conic decomposition.

Remark : In theorem 2.1.b we showed that the surface $X_{\checkmark}$ is non-singular if and only if the semigroup $\stackrel{\vee}{\sigma} \cap \mathbb{Z}^{2}$ is generated by a $\mathbb{Z}$-basis of $\mathbb{Z}^{2}$.

This shows that if $\sigma=\left\{\lambda\left(a e_{1}+b e_{2}\right)+\mu\left(c e_{1}+d e_{2}\right) \mid \lambda, \mu \in \mathbb{R}_{\geq 0}\right\}$ where $\left\{e_{1}, e_{2}\right\}$ is a basis of $\mathbb{Z}^{2}$ and $a, b, c, d \in \mathbb{Z}$ with g.c.d. $(a, b)=$ g.c.d. $(c, d)=1$ the surface $X_{\mathfrak{\sigma}}$ is non-singular if and only if $\operatorname{det}\left(\left(\begin{array}{ll}a & c \\ b & d\end{array}\right)\right)= \pm 1$.

Definition. We denote the rational ruled surfaces $\mathbb{P}(\mathcal{O}(m) \oplus \mathcal{O})$ by $\Sigma_{m}$. (See $\left.[\mathrm{H}]\right)$. Sometimes these surfaces are called the Hirzebruch surfaces in the literature.

Theorem 2.3.

a) A minimal regular conic decomposition corresponds with one of the following surfaces :

$$
\Sigma_{0}, \mathbb{P}_{\bar{K}}^{2}, \Sigma_{m}, m \geq 2
$$


b) Every non-minimal regular conic decomposition gives a non-singular surface which can be obtained from one of the surfaces above by a finite succession of blow ups.

Proof. These facts are proved in [0.1] and [0.2]. We will not recall the proof here. In the next remark we will describe the minimal regular conic decompositions.

Remark : In the pictures below we give the minimal regular conic decompositions and the surfaces defined by them.
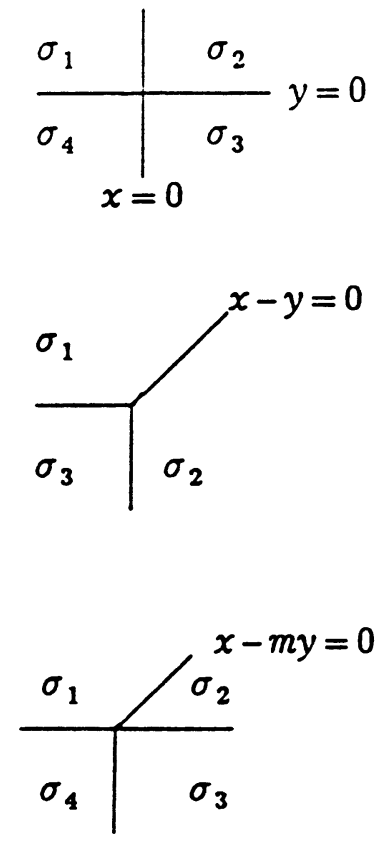

$$
\Sigma_{0}=\mathbb{P} \frac{1}{K} \times \mathbb{P} \frac{1}{K}
$$

$$
\mathbb{P}_{\bar{K}}^{2}
$$

$\Sigma_{m}, m \geq 2$

So a minimal regular conic decomposition consists of 3 or 4 cones as above.

If a regular conic decomposition $R_{1}$ is not minimal then there are two cones $\sigma_{i}$ and $\sigma_{j}$ in $R_{1}$ such that replacing $\sigma_{i}$ and $\sigma_{j}$ by their union $\sigma_{i} \cup \sigma_{j}$ gives another regular conic decomposition $R_{2}$. The surface defined by $R_{2}$ is a blowing down of the surface defined by $R_{1}$. By induction this proces stops if we reach a minimal regular conic decomposition as above. Below 
we show an example.

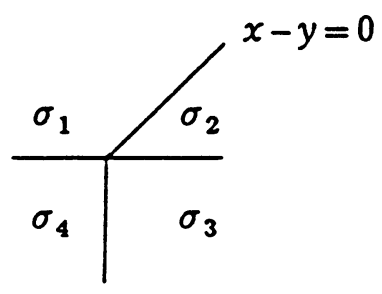

$$
R_{1} \text { defines a } \widetilde{\mathbb{P}}_{\bar{K}}^{2}=\Sigma_{1}
$$

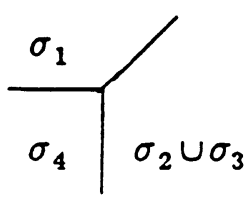

$$
R_{2} \text { defines a } \mathbb{P}_{\bar{K}}^{2}
$$

For later use we give the following decomposition :

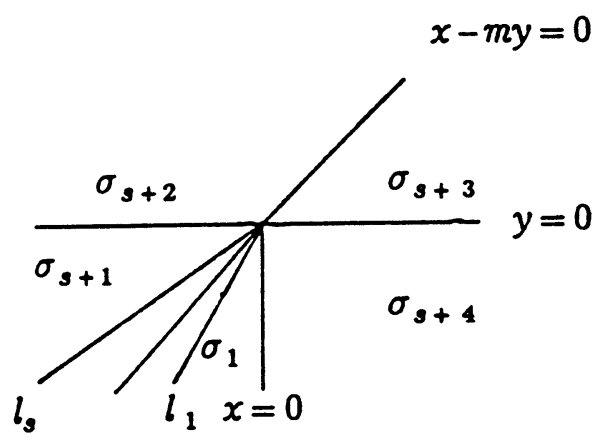


Here $l_{i}$ is given by $x-i y=0, i=1 \cdots s$. The regular conic decomposition $R_{s-i+1}$ is given by replacing $\sigma_{s+1}, \sigma_{s}, \cdots, \sigma_{i}$ by $\sigma_{s+1} \cup \sigma_{s} \cup \cdots \cup \sigma_{i}$. Since $R_{s}$ defines a surface $\Sigma_{m}, R_{0}$ defines a surface $\Sigma_{m}$ blown up $s$ times.

Theorem 2.4. Let $\Gamma$ be generated by a contraction $\gamma$. There exists a pure $\Gamma$-invariant affinoid covering $\mathcal{C}=\left(X_{i}\right)$ of $K^{2}-\{(0,0)\}$ where the $X_{i}$ are monomial rational domains such that every extremal point $P$ of $C_{i}=v\left(X_{i}\right)$ gives a non-singular surface in the reduction.

Proof. If $\left|\alpha_{1}\right|=\left|\alpha_{2}\right|$ we have proved this in lemma 2.5. All other cases are proved in the next proposition.

Proposition 2.3. Let $\Gamma$ be generated by a contraction $\gamma$ with $0<\left|\alpha_{1}\right|<\left|\alpha_{2}\right|<1$. Now there exists for every $l \in \mathbb{Z}_{>1}$ such that $\left|\alpha_{2}^{l}\right|<\left|\alpha_{1}\right|$ a pure affinoid covering as stated in theorem 2.4 above. The reduction is as shown in the figure below.

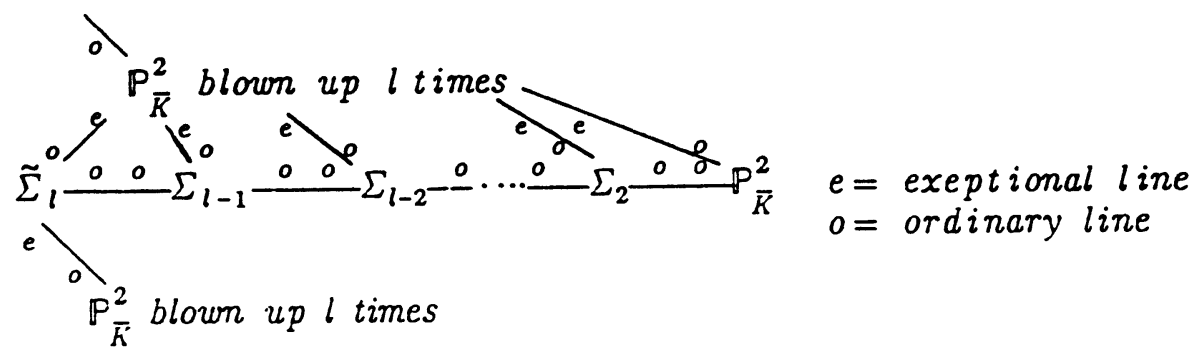

Proof. Let $F$ be the fundamental domain of $\Gamma$ constructed in proposition 2.1. Let $v$ denote as before the map $v:\left(z_{1}, z_{2}\right) \rightarrow\left(\log \left|z_{1}\right|, \log \left|z_{2}\right|\right)$.

We now look at $v(F)$. Let the point $\mathcal{Q}=\left(q_{1}, q_{2}\right) \in v(F)$ be defined by :

$$
\left\{\begin{array}{l}
x_{1}-x_{2}=0 \\
x_{1}-l x_{2}=\log \left|\alpha_{1}\right|-l \cdot \log \left|\alpha_{2}\right|
\end{array}\right.
$$

This point $\mathcal{Q}$ is in $v(F)$ since $0<\left|\alpha_{2}^{l}\right|<\left|\alpha_{1}\right|<\left|\alpha_{2}\right|<1$ and therefore we have

$$
0>q_{1}=q_{2}=\frac{\log \left|\alpha_{1}\right|-l \cdot \log \left|\alpha_{2}\right|}{1-l}>\log \left|\alpha_{2}\right|>\log \left|\alpha_{1}\right| .
$$


We now cover the area $v(F)$ by a finite number of convex domains as shown in the figure.

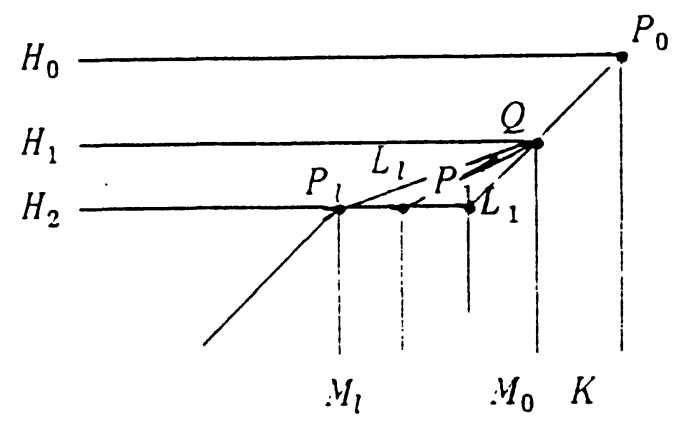

The line-segments drawn in the figure above are the following :

$$
\begin{array}{llll}
L_{1} & x_{1}-x_{2}=0 & x_{2} \in\left[\log \left|\alpha_{2}\right|, 0\right] & \\
L_{i} & x_{1}-i x_{2}=q_{1}-i q_{2} & x_{2} \in\left[\log \left|\alpha_{2}\right|, q_{2}\right] & i=2 \ldots l \\
M_{0} & x_{1}=q_{1} & x_{2} \in\left[-\infty, q_{2}\right] & \\
M_{i} & x_{1}=q_{1}-i q_{2}+i \log \left|\alpha_{2}\right| & x_{2} \in\left[-\infty, \log \left|\alpha_{2}\right|\right] & i=1 \ldots l \\
K & x_{1}=0 & x_{2} \in[-\infty, 0] & \\
H_{0} & x_{2}=0 & x_{1} \in[-\infty, 0] \\
H_{1} & x_{2}=q_{2} & x_{1} \in\left[-\infty, q_{1}\right] & \\
H_{2} & x_{2}=\log \left|\alpha_{2}\right| & x_{1} \in\left[-\infty, \log \left|\alpha_{2}\right|\right]
\end{array}
$$

The extremal points of the convex domains are $\mathcal{Q}, P_{i}, i=0 \ldots l$. The points $P_{i}$ are given by $P_{0}=(0,0)$ and $P_{i}=\left(q_{1}-i q_{2}+i \log \left|\alpha_{2}\right|, \log \left|\alpha_{2}\right|\right), i=1 \ldots l$.

Now proposition 2.2 tells us that the covering of $v(F)$ with convex domains as above corresponds with a covering of $F$ with monomial rational domains. Furthermore lemma 2.4 shows us that the covering of $K^{2}-\{(0,0)\}$ arising from this covering of the fundamental domain $F$ by the action of $\Gamma$ is a pure affinoid covering of $K^{2}-\{(0,0)\}$. 
The extremal points $\mathcal{Q}, P_{i}, i=0 . . l$ give surfaces in the reduction of $K^{2}-\{(0,0)\}$. The remark before theorem 2.4 shows that the surfaces are the following :

to $\mathcal{Q}$ belongs a $\mathbb{P}_{\bar{K}}^{2}$ blown up $l$ times.

to $P_{1}$ belongs a $\mathbb{P}_{\bar{K}}^{2}$.

to $P_{i}, i=2 \cdots l-1$ belongs a $\Sigma_{i}$.

to $P_{0}$ and $P_{l}=\gamma\left(P_{0}\right)$ belongs a $\tilde{\Sigma}_{l}$, i.e. a $\Sigma_{l}$ blown up one time.

The surfaces belonging to two different extremal points have at most one line in common. They have exactly one line $\mathbb{P} \frac{1}{K}$ in common if and only if the extremal points are joined by a line segment that is a part of the boundary of a convex domain. The line they have in common is defined by the monomials that reach their maximum on this rational line. A direct calculation shows that these lines are as in the statement of the proposition.

Proposition 2.4. Let $\Gamma$ be generated by a contraction $\gamma$ such that $\left|\alpha_{2}^{l}\right|=\left|\alpha_{1}\right|$ for some $l \in \mathbb{Z}_{>1}$. Now there exists a pure affinoid covering as stated in theorem 2.4 above. The reduction is shown in the figure below.

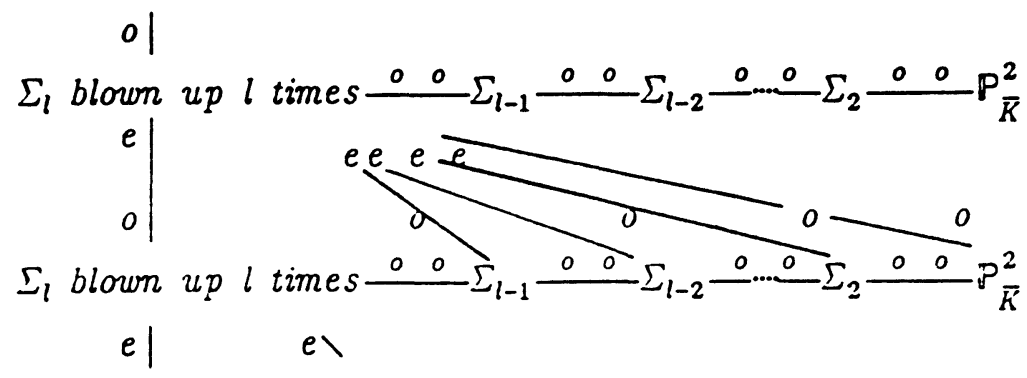

Proof. The construction of the covering is the same as in proposition 2.3. The only difference is that we have now $\mathcal{Q}=P_{0}$. Therefore $H_{1}=H_{0}$ and $K=M_{0}$. 
Looking at the figure below it is clear that we have a covering as in theorem 2.4 and that the reduction is as stated above.

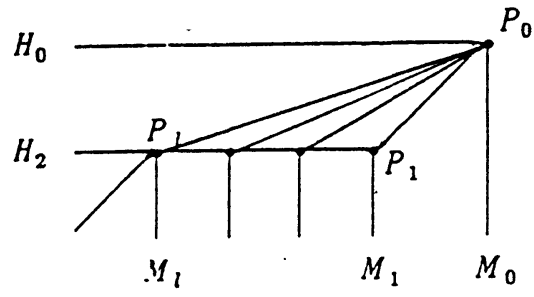

Remark. Using the coverings of $K^{2}-\{(0,0)\}$ given above, we can find a reduction of the Hopf surface $X=K^{2}-\{(0,0)\} / \Gamma$. Since $\Gamma$ covers $K^{2}-\{(0,0)\}$ with the images of the fundamental domain $F$, it is clear that the reduction of $X$ is as shown in the statements of the propositions 2.3 and 2.4 above with an identification of some lines.

We will now construct another example with $\Gamma=\langle\gamma, \tilde{\zeta}\rangle$ where $\gamma$ is the contraction $\gamma:\left(z_{1}, z_{2}\right) \rightarrow\left(\alpha_{1} z_{1}, \alpha_{2} z_{2}\right)$ and $\widetilde{\zeta}$ generates $\Gamma_{\text {tors }}, \widetilde{\zeta}$ is defined by $\widetilde{\zeta}:\left(z_{1}, z_{2}\right) \rightarrow\left(\zeta z_{1}, \zeta z_{2}\right)$ where $\zeta$ is a primitive $m$-th root of unity. In this case the Hopf surface $X=K^{2}-\{(0,0)\} / \Gamma$ has a nice reduction $\Sigma_{m} / \sim$, where $\sim$ is an equivalence relation identifying two lines of $\Sigma_{m}$.

LEMMA 2.6. Let $\zeta$ be a primitive $m$-th root of unity. Let $\langle\tilde{\zeta}\rangle$ be a group acting on $K^{2}-\{(0,0)\}$ where $\widetilde{\zeta}$ is defined by $\widetilde{\zeta}:\left(z_{1}, z_{2}\right) \rightarrow\left(\zeta z_{1}, \zeta z_{2}\right)$.

Let $\Sigma_{m}$ be as above with a homogeneous coordinate ring

$$
K\left[x_{1}, x_{2}\right]\left[z, y_{0}, . . y_{m}\right] / I,
$$

where $I$ is the ideal

$$
\left.I=\left\langle y_{i} x_{1}^{j} x_{2}^{m-j}-y_{j} x_{1}^{i} x_{2}^{m-i} \mid 0 \leq i, j \leq m\right\rangle \quad \text { (see }[H]\right) .
$$

Now we have : $K^{2}-\{(0,0)\} /\langle\tilde{\zeta}\rangle \simeq \Sigma_{m} \backslash S$.

Here $S$ consists of the two lines $\mathbb{P}_{K}^{1}$ defined by

$$
z=0 \text { and by } y_{0}=y_{1}=\cdots=y_{m}=0 .
$$

Proof. The group $\langle\tilde{\zeta}\rangle$ acts on the two open subspaces $K^{*} \times K$ of $K^{2}-\{(0,0)\}$ defined by $z_{1} \neq 0$ and $z_{2} \neq 0$. These two subspaces cover the 
whole of $K^{2}-\{(0,0)\}$. Now it is sufficient to look at the invariants of the group action on these two open subspaces. The invariants are generated by :

$$
\begin{cases}\frac{z_{2}}{z_{1}} \text { and } z_{1}^{m} \text { if } z_{1} \neq 0 \\ \frac{z_{1}}{z_{2}} \text { and } z_{2}^{m} \text { if } z_{2} \neq 0\end{cases}
$$

We will now glue the spaces defined by these coordinates together to find a description of $K^{2}-\{(0,0)\} /\langle\widetilde{\zeta}\rangle$. First we take homogeneous coordinates $x_{1}, x_{2}$ such that $\frac{x_{1}}{x_{2}}=\frac{z_{1}}{z_{2}}$ and $\frac{x_{2}}{x_{1}}=\frac{z_{2}}{z_{1}}$. Let $y_{i}, i=0 \ldots m$ be the nonhomogeneous coordinates $y_{i}=z_{1}^{i} z_{2}^{m-i}, i=0 \ldots m$. The $y_{i}$ are global sections of $K^{2}-\{(0,0)\} /<\widetilde{\zeta}>$. Together with $x_{1}$ and $x_{2}$ they give a complete description of the coordinate ring of $\left.K^{2}-\{(0,0)\} /<\widetilde{\zeta}\right\rangle$.

The coordinate ring is given by :

$$
R=K\left[x_{1}, x_{2}\right] \times K\left[y_{0}, \ldots, y_{m}\right] / I, I=\left\langle y_{i} x_{1}^{j} x_{2}^{m-j}-y_{j} x_{1}^{i} x_{2}^{m-i} \mid 0 \leq i, j \leq m\right\rangle
$$

Furthermore we have the condition that $y_{0}$ and $y_{m}$ cannot be both zero, coming from the fact that the point $(0,0)$ is missing in $K^{2}-\{(0,0)\}$.

Since the coordinate ring of $\Sigma_{m}$ is $K\left[x_{1}, x_{2}\right] \times K\left[z, y_{0}, \ldots, y_{m}\right] / I$ and $R \simeq K\left[x_{1}, x_{2}\right] \times K\left[1, y_{0}, \ldots, y_{m}\right] / I$, it is clear that $K^{2}-\{(0,0)\} /\langle\widetilde{\zeta}\rangle$ is isomorphic to a subspace of $\Sigma_{m}$.

In fact we have : $K^{2}-\{(0,0)\} /\langle\widetilde{\zeta}\rangle \simeq \Sigma_{m} \backslash S$.

Here $S$ consists of the two lines $\mathbb{P}_{K}^{1}$ defined by :

$$
\begin{cases}z=0 & K\left[x_{1}, x_{2}\right] \times K\left[0, y_{0}, \ldots y_{m}\right] / I \\ y_{0}=\ldots=y_{m}=0 & K\left[x_{1}, x_{2}\right] \times K[1,0 \ldots 0] / I\end{cases}
$$

Proposition 2.5. Let $\widetilde{\zeta}$ be as above. Let $\Gamma$ be the group $\Gamma=\langle\widetilde{\zeta}, \gamma\rangle$ acting on $K^{2}-\{(0,0)\}$. Here $\gamma$ is a contraction defined by

$$
\gamma:\left(z_{1}, z_{2}\right) \rightarrow\left(\alpha z_{1}, \alpha z_{2}\right) \text { with } 0<|\alpha|<1 .
$$

Now there exists a pure $\Gamma$-invariant affinoid covering of $K^{2}-\{(0,0)\}$ such that the Hopf surface $K^{2}-\{(0,0)\} / \Gamma$ has the reduction $\Sigma_{m} / \sim$. Here $\sim$ is the equivalence relation identifying the two lines $\mathbb{P} \frac{1}{K}$ defined by $z=0$ and by $y_{0}=y_{1}=\cdots=y_{m}=0$, where $z, y_{i}, i=0, \cdots, m$ are as in lemma 2.6. 
Proof. Since $\Gamma$ is abelian and $\langle\widetilde{\zeta}\rangle$ is finite, it is sufficient to find a fundamental domain $F$ for the action of $\left\langle\gamma>\right.$ on $K^{2}-\{(0,0)\} /\langle\widetilde{\zeta}\rangle$ such that the affinoid covering of $F$ together with its $\langle\gamma\rangle$-images is pure and gives a reduction with the properties mentioned above.

Again we consider the two open subspaces $K^{*} \times K$ of $K^{2}-\{(0,0)\}$ defined by $z_{1} \neq 0$ and by $z_{2} \neq 0$. It is clear that the action of $\gamma$ on the $<\widetilde{\zeta}>$-invariants is given by :

$$
\begin{cases}\gamma: \frac{z_{1}}{z_{2}} \rightarrow \frac{z_{1}}{z_{2}}, z_{2}^{m} \rightarrow \alpha^{m} z_{2}^{m} & \text { if } z_{2} \neq 0 \\ \gamma: \frac{z_{2}}{z_{1}} \rightarrow \frac{z_{2}}{z_{1}}, z_{1}^{m} \rightarrow \alpha^{m} z_{1}^{m} & \text { if } \quad z_{1} \neq 0 .\end{cases}
$$

Now we have a fundamental domain $F=F_{1} \cup F_{2}$ where $F_{1}, F_{2}$ are given by :

$$
\begin{aligned}
& F_{1}=\left\{\left(\frac{z_{1}}{z_{2}}, z_{2}^{m}\right)|| \alpha^{m}|\leq| z_{2}^{m}|\leq 1,| \frac{z_{1}}{z_{2}} \mid \leq 1\right\} \\
& F_{2}=\left\{\left(\frac{z_{2}}{z_{1}}, z_{1}^{m}\right)|| \alpha^{m}|\leq| z_{1}^{m}|\leq 1,| \frac{z_{2}}{z_{1}} \mid \leq 1\right\} .
\end{aligned}
$$

The subspaces $F_{1}$ and $F_{2}$ are affinoid spaces, they are in fact monomial rational domains. It is easy to see that $\mathcal{C}=\left\{\gamma^{i}\left(F_{1}\right), \gamma^{j}\left(F_{2}\right) \mid i, j, \in \mathbb{Z}\right\}$ is a pure affinoid covering of $K^{2}-\{(0,0)\} /\langle\widetilde{\zeta}\rangle$ by monomial rational domains.

We will now define maps $v^{\prime}$ and $v^{\prime \prime}$ such that $v^{\prime}\left(\gamma^{i}\left(F_{1}\right)\right)$ and $v^{\prime \prime}\left(\gamma^{i}\left(F_{2}\right)\right)$ are convex domains in $(\mathbb{R} \cup\{ \pm \infty\})^{2}$. The maps are defined by :

$$
\begin{aligned}
& v^{\prime}:\left\{\left(\frac{z_{1}}{z_{2}}, z_{2}^{m}\right) \in K^{2}-\{(0,0)\} /<\widetilde{\zeta}>\mid z_{2} \neq 0\right\} \rightarrow(\mathbb{R} \cup\{ \pm \infty\})^{2}, \\
& v^{\prime}\left(\frac{z_{1}}{z_{2}}, z_{2}^{m}\right)=\left(\log \left|z_{2}^{m}\right|, \log \left|\frac{z_{1}}{z_{2}}\right|\right) . \\
& v^{\prime \prime}:\left\{\left(\frac{z_{2}}{z_{1}}, z_{1}^{m}\right) \in K^{2}-\{(0,0)\} /<\widetilde{\zeta}>\mid z_{1} \neq 0\right\} \rightarrow(\mathbb{R} \cup\{ \pm \infty\})^{2}, \\
& v^{\prime \prime}\left(\frac{z_{2}}{z_{1}}, z_{1}^{m}\right)=\left(m \log \left|\frac{z_{2}}{z_{1}}\right|+\log \left|z_{1}^{m}\right|,-\log \left|\frac{z_{2}}{z_{1}}\right|\right) .
\end{aligned}
$$

Since the maps $v^{\prime}$ and $v^{\prime \prime}$ are identical on the subspace defined by $z_{1} \neq 0, z_{2} \neq 0$, we can glue them together and get a map

$$
v: K^{2}-\{(0,0)\} /<\widetilde{\zeta}>\rightarrow(\mathbb{R} \cup\{ \pm \infty\})^{2} .
$$




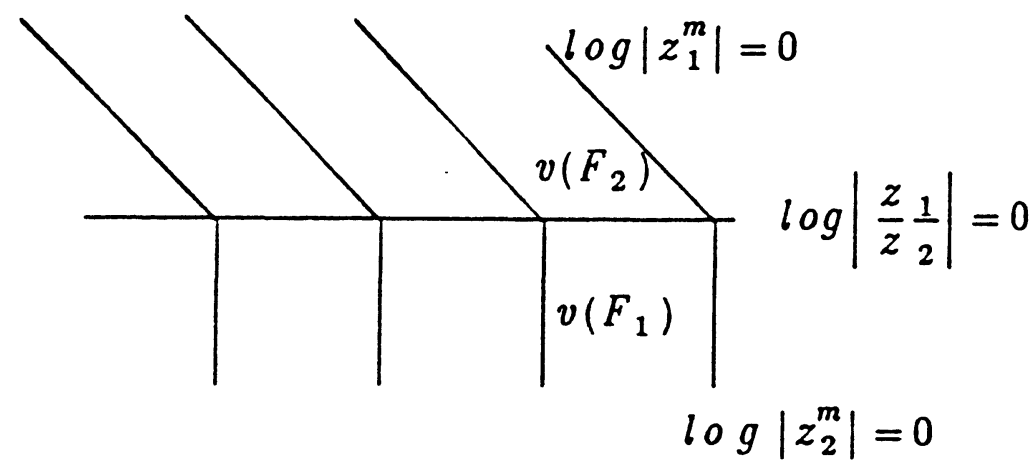

Note that in this situation $v\left(F_{2}\right)$ does not satisfy condition $b$ of proposition 2.2. This is a consequence of the definition of $v$ used here. But the results of lemma 2.4 remain valid, mutatis mutandis.

Again we have a 1-1 correspondence between extremal points $P$ of the convex domains $v\left(\gamma^{i}\left(F_{j}\right)\right), i \in \mathbb{Z}, j=1,2$ and the components of the reduction. Every extremal point $P$ gives a surface in the reduction. Looking at the figure above and using the remark following theorem 2.3 we see that every extremal point $P$ gives a surface $\Sigma_{m}$.

In order to describe the intersections of the surfaces $\Sigma_{m}$ we need some more information. We need to know the components in $P$ of the reduction of the four affinoid domains $v\left(\gamma^{i}\left(F_{j}\right)\right)$ with $P \in v\left(\gamma^{i}\left(F_{j}\right)\right.$ in $P$. This is a straightforward calculation. The results are shown in the figure below. 

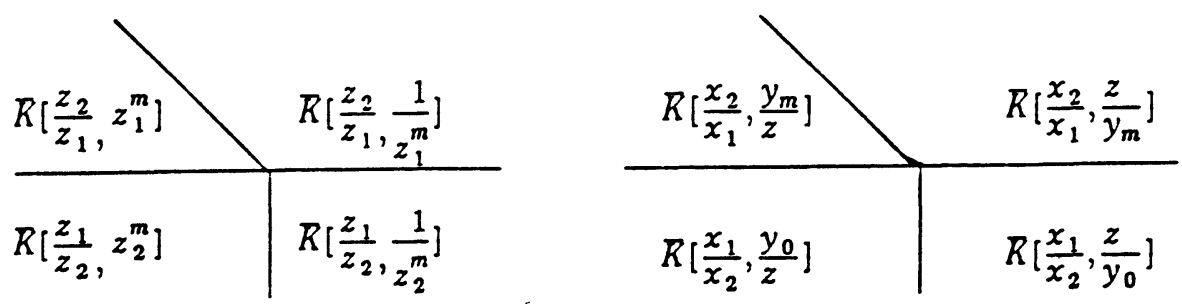

The components can be glued together to give a surface $\Sigma_{m}$ with homogeneous coordinate ring $\bar{K}\left[x_{1}, x_{2}\right] \times \bar{K}\left[z, y_{0}, \ldots, y_{m}\right] / I$. The identification is given by :

$$
\frac{x_{2}}{x_{1}}=\frac{z_{2}}{z_{1}}, \frac{x_{1}}{x_{2}}=\frac{z_{1}}{z_{2}}, \frac{y_{m}}{z}=z_{1}^{m}, \frac{y_{0}}{z}=z_{2}^{m} \text { etc. }
$$

Now it is clear from the figures above that the surfaces $\Sigma_{m}$ belonging to the extremal points $P$ and $\gamma(P)$ have a line in common. This line is defined by $\frac{y_{m}}{z}=\frac{y_{0}}{z}=0$ in the $\Sigma_{m}$ belonging to $P$ and by $\frac{z}{y_{m}}=\frac{z}{y_{0}}=0$ in the other $\Sigma_{m}$. So these lines are $\mathbb{P} \frac{1}{K}$ 's defined by $z=0$ and by $y_{0}=y_{1}=\ldots=y_{m}=0$.

Since $\langle\gamma\rangle$ is transitive on the set of extremal points $P$ it is clear that the reduction of the Hopf surface $K^{2}-\{(0,0)\} / \Gamma$ is $\Sigma_{m} / \sim$, where $\sim$ identifies the two lines above.

\section{Line bundles on a Hopf surface}

Let $\Gamma=\langle\gamma\rangle$ be generated by a contraction $\gamma$. We will now study the line bundles on the Hopf surface $X=K^{2}-\{(0,0)\} / \Gamma$. We will need some properties of quasi-Stein spaces (see [Ki.2]).

Definition. An analytic space $Y$ is called a quasi-Stein space if there exists an admissible covering of $Y$ by open affinoid subspaces $U_{1}, U_{2}, U_{3}, \ldots$ such that :

1) $U_{1} \subseteq U_{2} \subseteq U_{3} \subseteq$...

2) the image of $\mathcal{O}_{Y}\left(U_{i+1}\right)$ is dense in $\mathcal{O}_{Y}\left(U_{i}\right)$.

Remark : Let $Y$ be a quasi-Stein space and $\mathcal{F}$ a coherent sheaf on $Y$. In [Ki.2] the following two properties are proved :

1) $H^{i}(Y, \mathcal{F})=0 \forall i>0$

2) The coherent sheaf $\mathcal{F}$ is a sheaf associated with an $\mathcal{O}(Y)$-module $F$. 
Lemma 3.1. The analytic space $K^{*} \times K$ is a quasi-Stein space.

Proof. Let $U_{i}$ be the open affinoid subspace of $K^{*} \times K$ defined by :

$$
R_{i}^{-1} \leq\left|z_{1}\right| \leq R_{i},\left|z_{2}\right| \leq R_{i}
$$

We choose $R_{i}<R_{i+1}$ and $R_{i} \rightarrow \infty$ for $i \rightarrow \infty$.

Now the covering $\left(U_{i}\right)_{i \in \mathbb{N}}$ of $K^{*} \times K$ is admissible and satisfies the definition above. This proves the lemma.

Lemma 3.2. Every line bundle $\mathfrak{L}$ on $W=K^{2}-\{(0,0)\}$ is trivial.

Proof. We have $W=W_{1} \cup W_{2}$ where $W_{1}=\left\{\left(z_{1}, z_{2}\right) \in W \mid z_{1} \neq 0\right\}$ and $W_{2}=\left\{\left(z_{1}, z_{2}\right) \in W \mid z_{2} \neq 0\right\}$. Now $\left.\mathfrak{L}\right|_{W_{i}} \simeq \mathcal{O}_{W_{i}} \cdot e_{i}$, since $W_{i} \simeq K^{*} \times K$ is a quasi-Stein space and every line bundle on $K^{*} \times K$ is trivial.

It is clear that

$$
\mathcal{O}\left(W_{1}\right)^{*}=K^{*} z_{1}^{Z_{1}}, \mathcal{O}\left(W_{2}\right)^{*}=K^{*} z_{2}^{\not / A} \text { and } \mathcal{O}\left(W_{1} \cap W_{2}\right)^{*}=K^{*} z_{1}^{\not Z} z_{2}^{Z_{1}}
$$

This shows that $e_{1}=a e_{2}$ for some $a \in \mathcal{O}\left(W_{1} \cap W_{2}\right)^{*}$. Furthermore we have $a=a_{1}^{-1} \cdot a_{2}$ with $a_{i} \in \mathcal{O}\left(W_{i}^{*}\right)$.

Now we take $f_{1}=a_{1} e_{1}$ and $f_{2}=a_{2} e_{2}$. Clearly we have $\left.\mathfrak{L}\right|_{W_{1}}=\mathcal{O}_{W_{i}} \cdot f_{i}$ and $\left.f\right|_{W_{i}}=f_{i}$ and $\mathfrak{L}=\mathcal{O}_{W_{i}} \cdot f$. This proves the lemma.

Remark : Let $u$ be the map $u: W=K^{2}-\{(0,0)\} \rightarrow W / \Gamma$ and let $\mathfrak{L}$ be a line bundle on $X=W / \Gamma$. Now $u^{*} \mathfrak{L}$ is a line bundle on $W$, so we have $u^{*} \mathfrak{L}=\mathcal{O}_{W} \cdot e$. The action of the contraction $\gamma$ on $u^{*} \mathfrak{L}$ has the form $\gamma(e)=\alpha \cdot e$ for some $\alpha \in \mathcal{O}_{W}(W)^{*}$. Clearly we have $: \mathcal{O}_{W}(W)^{*}=K^{*}$.

Definition. For $\alpha \in K^{*}$ we denote by $\mathfrak{L}_{\alpha}$ the line bundle on $X=W / \Gamma$ defined by $u^{*} \mathfrak{L}_{\alpha}=\mathcal{O}_{W} \cdot e$ with $\gamma(e)=\alpha \cdot e$. Here $\gamma$ is the contraction generating $\Gamma$.

Proposition 3.1. Let $\Gamma$ be generated by a contraction $\gamma$ and let $X=W / \Gamma$. Now we have :
a) Every line bundle $\mathfrak{L}$ on $X$ is isomorphic to an unique $\mathfrak{L}_{\alpha}$.
b) $\mathfrak{L}_{\alpha} \otimes \mathfrak{L}_{\beta} \simeq \mathfrak{L}_{\alpha \beta}$
c) $\operatorname{Pic}(X) \simeq K^{*}$ 
Proof. This is a direct consequence of lemma 3.2 and the remark following that lemma. Another way to prove the proposition is the following. The map $u: W \rightarrow X$ is a local isomorphism of the Grothendieck topology. We have :

$$
u^{*} \mathcal{O}_{X}^{*}=\mathcal{O}_{W}^{*} \text { and } H^{0}\left(X, \mathcal{O}_{X}^{*}\right)=H^{0}\left(W, u^{*} \mathcal{O}_{X}^{*}\right)^{\Gamma}
$$

Therefore we have : $H^{1}\left(X, \mathcal{O}_{X}^{*}\right)=H^{1}\left(\Gamma, H^{0}\left(W, \mathcal{O}_{W}^{*}\right)\right)=H^{1}\left(\Gamma, K^{*}\right)$. Since $\Gamma$ has trivial action on $K^{*}$ we see that $H^{1}\left(\Gamma, K^{*}\right)=K^{*}$. This shows that : $\operatorname{Pic}(X)=H^{1}\left(X, \mathcal{O}_{X}^{*}\right)=K^{*}$.

Remark : In the next lemma we shall compute $H^{i}\left(W, u^{*} \mathfrak{L}_{\alpha}\right) \simeq H^{i}\left(W, \mathcal{O}_{W}\right)$. We will need this for the calculation of the dimension of the groups $H^{i}\left(X, \mathfrak{L}_{\alpha}\right)$.

Lemma 3.3. Let $W=K^{2}-\{(0,0)\}$. The cohomology groups $H^{i}\left(W, \mathcal{O}_{W}\right)$ are given by :

$$
\begin{aligned}
& H^{0}\left(W, \mathcal{O}_{W}\right)=\left\{\sum_{n, m \geq 0} a_{n, m} z_{1}^{n} z_{2}^{m} \mid \text { the powerseries are converging on } W\right\} \\
& H^{1}\left(W, \mathcal{O}_{W}\right)=\left\{\begin{array}{r}
\sum_{n, m<0} a_{n, m} z_{1}^{n} z_{2}^{m} \mid \text { the powerseries are convergent on } \\
W \backslash\left\{\left(z_{1} z_{2}\right) \mid z_{1}=0 \text { or } z_{2}=0\right\}
\end{array}\right\} \\
& H^{i}\left(W, \mathcal{O}_{W}\right)=0, i \geq 2 .
\end{aligned}
$$

Proof. Let $W_{i} \simeq K^{*} \times K$ be the subspace of $W$ given by $z_{i} \neq 0$ for $i=1,2$. We have $W=W_{1} \cup W_{2}$. Since $W_{i}$ is a quasi-Stein space, we have $H^{j}\left(W_{i}, \mathfrak{L}\right)=0$ for $j>0$ and every coherent sheaf $\mathfrak{L}$ on $W_{i}$. Therefore we can use Leray's theorem.

Let $d$ be the natural map $d: \mathfrak{L}\left(W_{1}\right) \otimes \mathfrak{L}\left(W_{2}\right) \rightarrow \mathfrak{L}\left(W_{1} \cap W_{2}\right)$. Now Leray's theorem gives us : $H^{0}(W, \mathfrak{L})=\operatorname{ker} d, H^{1}(W, \mathfrak{L})=$ coker $d$ and 
$\operatorname{dim} H^{i}(W, \mathfrak{L})=0, i \geq 2$. Now the take $\mathfrak{L}=\mathcal{O}_{W}$. It is clear that we have :

$$
\begin{aligned}
& \mathfrak{L}\left(W_{1}\right)=\left\{\sum_{n \in Z, m \geq 0} a_{n, m} z_{1}^{n} z_{2}^{m} \mid \text { the } \text { powerseries converges on } W_{1}\right\}, \\
& \mathfrak{L}\left(W_{2}\right)=\left\{\sum_{\substack{n \geq 0, m \in \mathbb{Z}}} b_{n, m} z_{1}^{n} z_{2}^{m} \mid \text { the powerseries converges on } W_{2}\right\}, \\
& \mathfrak{L}\left(W_{1} \cap W_{2}\right)=\left\{\begin{array}{l}
\sum_{n, m \in \mathbb{Z}} c_{n, m} z_{1}^{n} z_{2}^{m} \mid \text { the powerseries converges on } \\
W_{1} \cap W_{2}
\end{array}\right\} .
\end{aligned}
$$

Now the lemma is proved by applying Leray's theorem.

Remark : Let $M$ be a $\Gamma$-module and let $\Gamma$ be $\Gamma=<\gamma>\simeq \mathbb{Z}$. Let $d: M \rightarrow M$ be the map given by $d(m)=\gamma(m)-m, m \in M$. Then the groups $H^{i}(\Gamma, M)$ are given by :

$$
\begin{aligned}
& H^{0}(\Gamma, M)=\text { ker } d \\
& H^{1}(\Gamma, M)=\text { coker } d \\
& H^{i}(\Gamma, M)=0, i \geq 2 .
\end{aligned}
$$

Proposition 3.2. Let $X=W / \Gamma, \Gamma \simeq \mathbb{Z}$ and $\mathfrak{L}_{\alpha}$ a line bundle on the Hopf surface $X$. In this situation we have :

a) $H^{0}\left(X, \mathfrak{L}_{\alpha}\right) \simeq H^{0}\left(\Gamma, H^{0}\left(W, u^{*} \mathfrak{L}_{\alpha}\right)\right)$

b) $0 \rightarrow H^{1}\left(\Gamma, H^{0}\left(W, u^{*} \mathfrak{L}_{\alpha}\right)\right) \rightarrow H^{1}\left(X, \mathfrak{L}_{\alpha}\right) \rightarrow H^{0}\left(\Gamma, H^{1}\left(W, u^{*} \mathfrak{L}_{\alpha}\right)\right) \rightarrow 0$ is exact.

c) $H^{2}\left(X, \mathfrak{L}_{\alpha}\right) \simeq H^{1}\left(\Gamma, H^{1}\left(W, u^{*} \mathfrak{L}_{\alpha}\right)\right)$

Proof. It is clear that $H^{0}\left(X, \mathfrak{L}_{\alpha}\right)=H^{0}\left(W, u^{*} \mathfrak{L}_{\alpha}\right)^{\Gamma}=H^{0}\left(\Gamma, H^{0}\left(W, u^{*} \mathfrak{L}_{\alpha}\right)\right)$. We will use spectral sequences to determine the other groups $H^{i}\left(X, \mathfrak{L}_{\alpha}\right)$.

The left exact functor $H^{*}(X,-)$ is the composition of the two left exact functors $H^{0}(\Gamma,-)$ and $H^{0}(W,-)$ and the exact functor $\mathfrak{L}_{\alpha} \rightarrow u^{*} \mathfrak{L}_{\alpha}$. We can determine the right derived functors $H^{i}(X,-)$ of $H^{0}(X,-)$ by using the right derived functors $H^{i}(\Gamma,-)$ and $H^{i}(W,-)$ of $H^{0}(\Gamma,-)$ and $H^{0}(W,-)$.

Let $T, U$ be covariant functors in one variable. Now [CE] p.376 gives us for the composite functor $V=T U$ a spectral sequence $\Pi_{r}^{p, q} \Longrightarrow \mathcal{R}^{n} T U(-)$. 
Here $\mathcal{R}^{n}$ is the $n-t h$ right derived functor. In this spectral sequence we have $\Pi_{2}^{p, q}=\mathcal{R}^{q} T\left(\mathcal{R}^{p} U(-)\right)$. In our case we have $V=H^{0}(X,-), T=H^{0}(\Gamma,-)$ and $U=H^{0}(W,-)$.

This gives us :

$$
\begin{aligned}
& \Pi_{r}^{p, q} \Longrightarrow H^{n}\left(X \mathfrak{L}_{\alpha}\right), n=p+q \\
& \Pi_{2}^{p, q}=H^{q}\left(\Gamma, H^{p}\left(W, u^{*} \mathfrak{L}_{\alpha}\right)\right) .
\end{aligned}
$$

Furthemore we have $H^{q}(\Gamma,-)=0, q \neq 0,1$ and $H^{p}(W,-)=0, p \neq 0,1$. Therefore $\Pi_{2}^{p, q}=0, p, q \neq 0,1$. Now we have $\Pi_{2}^{p, q}=\Pi_{r}^{p, q} \forall r \geq 2$, since $d_{r}: \Pi_{r}^{p, q} \rightarrow \Pi_{r}^{p-r+1, q+r}$ is trivial for $r \geq 2$, i.e $d_{r} \equiv 0$, and the spectral sequence is defined by $H\left(\Pi_{r}\right)=\Pi_{r+1}$.

Since $\Pi_{2}^{p, q}=0, p, q \neq 0,1$ we have an exact sequence (See [CE] p.333) :

$$
0 \rightarrow \Pi_{2}^{0,1} \rightarrow H^{1}\left(X, \mathfrak{L}_{\alpha}\right) \rightarrow \Pi_{2}^{1,0} \rightarrow 0 .
$$

This is the exact sequence in part $b$ of the proposition. Furthermore we have : $H^{2}\left(X, \mathfrak{L}_{\alpha}\right) \cong \Pi_{2}^{1,1}=H^{1}\left(\Gamma, H^{1}\left(W, u^{*} \mathfrak{L}_{\alpha}\right)\right)$. This proves part $c$ of the proposition.

Lemma 3.4. Let $\gamma$ be a contraction given by $\gamma\left(z_{1}, z_{2}\right)=\left(\alpha_{1} z_{1}, \alpha_{2} z_{2}\right)$, $0<\left|\alpha_{1}\right| \leq\left|\alpha_{2}\right|<1$ and $\alpha_{1}^{k} \neq \alpha_{2}^{l} \forall k, l \in \mathbb{Z}$.

Let $\mathfrak{L}_{\beta}$ be a line bundle on $X=W / \Gamma$, where $\Gamma=\langle\gamma\rangle$. Then we have :

$$
\begin{aligned}
\operatorname{dim} H^{0}\left(X, \mathfrak{L}_{\beta}\right) & =\left\{\begin{array}{l}
1 \text { if } \beta=\alpha_{1}^{a} \alpha_{2}^{b}, a, b \in \mathbb{Z}_{\leq 0} \\
0 \text { in all other cases }
\end{array}\right. \\
\operatorname{dim} H^{1}\left(X, \mathfrak{L}_{\beta}\right) & =\left\{\begin{array}{l}
1 \text { if } \beta=\alpha_{1}^{a} \alpha_{2}^{b}, a, b \cdot \in \mathbb{Z} \\
0 \text { in all other cases }
\end{array}\right. \\
\operatorname{dim} H^{2}\left(X, \mathfrak{L}_{\beta}\right) & =\left\{\begin{array}{l}
1 \text { if } \beta=\alpha_{1}^{a} \alpha_{2}^{b}, a, b \in \mathbb{Z}_{>0} \\
0 \text { in all other cases }
\end{array}\right.
\end{aligned}
$$

Proof. The element $\gamma$ multiplies $a_{n, m} z_{1}^{n} z_{2}^{m} e \in \mathfrak{L}_{\beta}$ by a constant :

$$
\gamma\left(a_{n, m} z_{1}^{n} z_{2}^{m} e\right)=\alpha_{1}^{n} \alpha_{2}^{m} \beta \cdot a_{n, m} z_{1}^{n} z_{2}^{m} e .
$$

Since $\alpha_{1}^{k} \neq \alpha_{2}^{l} \forall k, l \in \mathbb{Z}$ we have :

$$
f e \in H^{i}\left(W, u^{*} \mathfrak{L}_{\beta}\right), \gamma(f e)=f e \Longrightarrow f=a_{n, m} z_{1}^{n} z_{2}^{m}, \alpha_{1}^{n} \alpha_{2}^{m} \beta=1 .
$$


From $H^{0}\left(X, \mathfrak{L}_{\beta}\right)=H^{0}\left(\Gamma, H^{0}\left(W, u^{*} \mathfrak{L}_{\beta}\right)\right)$ we can directly conclude that :

$$
\operatorname{dim} H^{0}\left(X, \mathfrak{L}_{\beta}\right)=1 \Leftrightarrow \beta=\alpha_{1}^{-a} \alpha_{2}^{-b}, a, b \in \mathbb{Z}_{\geq 0} .
$$

Indeed all monomials in $H^{0}\left(W, u^{*} \mathfrak{L}_{\beta}\right)$ are of the form $z_{1}^{n} z_{2}^{m}, n, m \in \mathbb{Z}_{\geq 0}$.

In a similar way we can determine $\operatorname{dim} H^{0}\left(\Gamma, H^{1}\left(W, u^{*} \mathfrak{L}_{\beta}\right)\right)$. From the action of $\gamma$ on $H^{i}\left(W, u^{*} \mathfrak{L}_{\beta}\right)$ we can see that :

$$
\operatorname{dim} H^{0}\left(\Gamma, H^{i}\left(W, u^{*} \mathfrak{L}_{\beta}\right)\right)=\operatorname{dim} H^{1}\left(\Gamma, H^{i}\left(W, u^{*} \mathfrak{L}_{\beta}\right)\right) .
$$

Now we can calculate

$\operatorname{dim} H^{2}\left(X, \mathfrak{L}_{\beta}\right)=\operatorname{dim} H^{1}\left(\Gamma, H^{1}\left(W, u^{*} \mathfrak{L}_{\beta}\right)\right)=\operatorname{dim} H^{0}\left(\Gamma, H^{1}\left(W, u^{*} \mathfrak{L}_{\beta}\right)\right)$ as before.

Now proposition $3.2 b$ shows us that :

$$
\begin{aligned}
\operatorname{dim} H^{1}\left(X, \mathfrak{L}_{\beta}\right) & =\operatorname{dim} H^{0}\left(\Gamma, H^{1}\left(W, u^{*} \mathfrak{L}_{\beta}\right)\right)+\operatorname{dim} H^{1}\left(\Gamma, H^{0}\left(W, u^{*} \mathfrak{L}_{\beta}\right)\right) \\
& =\operatorname{dim} H^{2}\left(X, \mathfrak{L}_{\beta}\right)+\operatorname{dim} H^{0}\left(X, \mathfrak{L}_{\beta}\right) .
\end{aligned}
$$

This gives us : $\operatorname{dim} H^{1}\left(X, \mathfrak{L}_{\beta}\right)=1 \Leftrightarrow \beta=\alpha_{1}^{a} \alpha_{2}^{b}, a, b \in \mathbb{Z}$

Lemma 3.5. Let $\gamma$ be a contraction given by $\gamma\left(z_{1}, z_{2}\right)=\left(\alpha_{1} z_{1}, \alpha_{2} z_{2}\right)$, $0<\left|\alpha_{1}\right| \leq\left|\alpha_{2}\right|<1$ and $\alpha_{1}^{k}=\alpha_{2}^{l}$ for some $k, l \in \mathbb{Z}_{>0}$ with g.c.d. $(k, l)=1$. Let $\mathfrak{L}_{\beta}$ be a line bundle on the Hopf surface $X=W / \Gamma$, where $\Gamma=\langle\gamma\rangle$. Now we have :

$$
\begin{aligned}
& \operatorname{dim} H^{0}\left(X, \mathfrak{L}_{\beta}\right)=\left\{\begin{array}{llr}
b+1 & \text { if } \beta=\alpha_{1}^{-r} \alpha_{2}^{-b l-s}, 0 \leq r<k, 0 \leq s<l, \\
0 & \text { in all other cases } & b \in \mathbb{Z}_{\geq 0}
\end{array}\right. \\
& \left\{\begin{array}{rr}
b+1 \quad \text { if } \beta=\alpha_{1}^{-r} \alpha_{2}^{-b l-s}, 0 \leq r<k, 0 \leq s<l, \\
b \in \mathbb{Z}_{\geq 0}
\end{array}\right. \\
& \operatorname{dim} H^{1}\left(X, \mathfrak{L}_{\beta}\right)=\left\{\begin{array}{r}
b+1 \quad \text { if } \beta=\alpha_{1}^{r} \alpha_{2}^{b l+s}, 0<r \leq k, 0<s \leq l, \\
\quad b \in \mathbb{Z}_{\geq 0}
\end{array}\right. \\
& 0 \quad \text { in all other cases } \\
& \operatorname{dim} H^{2}\left(X, \mathfrak{L}_{\beta}\right)= \begin{cases}b+1 & \text { if } \beta=\alpha_{1}^{r} \alpha_{2}^{b l+s}, 0<r \leq k, 0<s \leq l, \\
0 & \text { in all other cases. }\end{cases}
\end{aligned}
$$


Proof. As in the previous lemma we have for monomials $a_{n m} z_{1}^{n} z_{2}^{m} e \in H^{i}\left(W, u^{*} \mathfrak{L}_{\beta}\right)$ :

$$
\gamma\left(a_{n, m} z_{1}^{n} z_{2}^{m} e\right)=\alpha_{1}^{n} \alpha_{2}^{m} \beta \cdot a_{n, m} z_{1}^{n} z_{2}^{m} e .
$$

So an element $f \cdot e \in H^{i}\left(W, u^{*} \mathfrak{L}_{\beta}\right)$ is $\gamma$-invariant if and only if $f e$ is the sum of monomials $a_{n, m} z_{1}^{n} z_{2}^{m} \cdot e$ that are $\gamma$-invariant.

Now we can calculate $H^{0}\left(\Gamma, H^{i}\left(W, u^{*} \mathfrak{L}_{\beta}\right)\right)$. Since

$$
H^{0}\left(X, \mathfrak{L}_{\beta}\right)=H^{0}\left(\Gamma, H^{0}\left(W, u^{*} \mathfrak{L}_{\beta}\right)\right)
$$

we have $\operatorname{dim} H^{0}\left(X, \mathfrak{L}_{\beta}\right) \neq 0$ if and only if $\beta=\alpha_{1}^{-m} \alpha_{2}^{-n}, m, n \in \mathbb{Z}_{\geq 0}$. If $\beta=\alpha_{1}^{-m} \alpha_{2}^{-n}$ then we can write $\beta$ uniquely in the form $\beta=\alpha_{1}^{-r} \alpha_{2}^{-b l-s}$ with $0 \leq r<k, 0 \leq s<l$ since $\alpha_{1}^{k}=\alpha_{2}^{l}$.

Now we have $\beta=\alpha_{1}^{-m} \alpha_{2}^{-n}, m, n \in \mathbb{Z}_{\geq 0}$ for the following values of $m$ and $n$ :

$$
\left\{\begin{array}{l}
m=r+j k \\
n=s+b l-j l, 0 \leq j \leq b
\end{array}\right.
$$

This proves that $\operatorname{dim} H^{0}\left(X, \mathfrak{L}_{\beta}\right)=b+1$.

In a similar way we can calculate $\operatorname{dim} H^{0}\left(\Gamma, H^{1}\left(W, u^{*} \mathfrak{L}_{\beta}\right)\right)$. Since $\gamma$ acts on the space $H^{i}\left(W, u^{*} \mathfrak{L}_{\beta}\right)$ by multiplying the monomials with a constant, we have $: \operatorname{dim} H^{0}\left(\Gamma, H^{i}\left(W, u^{*} \mathfrak{L}_{\beta}\right)\right)=\operatorname{dim} H^{1}\left(\Gamma, H^{i}\left(W, u^{*} \mathfrak{L}_{\beta}\right)\right)$.

Now using proposition 3.2. it is straightforward to calculate $\operatorname{dim} H^{1}\left(X, \mathfrak{L}_{\beta}\right)$ and $\operatorname{dim} H^{2}\left(X, \mathfrak{L}_{\beta}\right)$.

Remark : Let $\gamma$ be a contraction of the form $\gamma\left(z_{1}, z_{2}\right)=\left(\alpha^{m}\left(z_{1}+\lambda z_{2}^{m}\right), \alpha z_{2}\right)$, $0<|\alpha|<1, \lambda \neq 0$. In this case $\gamma\left(W_{1}\right) \neq W_{1}$. So the action of $\gamma$ on $H^{1}\left(W, u^{*} \mathfrak{L}_{\beta}\right)$ as given in lemma 3.3 is not well-defined. Therefore we need another description of $H^{1}\left(W, u^{*} \mathfrak{L}_{\beta}\right)$. This will be done in the next lemma.

Lemma 3.6. Let $W_{1}^{\prime} \subset W$ be the subspace

$$
W_{1}^{\prime}=\left\{\left(z_{1}, z_{2}\right) \in W|| z_{1}|>| z_{2}^{m} \mid\right\} .
$$

Then $W_{1}^{\prime}$ is a quasi-Stein space and $W=W_{1}^{\prime} \cup W_{2}$. Let $\gamma$ be a contraction of the form

$$
\gamma\left(z_{1}, z_{2}\right)=\left(\alpha^{m}\left(z_{1}+\lambda z_{2}^{m}\right), \alpha z_{2}\right), 0<|\alpha|<1,0<|\lambda| \leq 1, m \in \mathbb{Z}_{>0} .
$$


We have

$H^{1}\left(W, \mathcal{O}_{W}\right) \simeq\left\{\sum_{n, m<0} a_{n, m} z_{1}^{n} z_{2}^{m} \mid\right.$ the powerseries converges on $\left.W_{1}^{\prime} \cap W_{2}\right\}$

The action of $\gamma$ on $H^{1}\left(W, \mathcal{O}_{W}\right)$ is now well-defined.

Proof. We can see that $W_{1}^{\prime}$ is a quasi-Stein space by using the following admissible affinoid covering $\left(U_{i}\right)_{i \in \mathbb{R}_{\geq 0}}$ of $W_{1}^{\prime}$. Here $U_{i}$ is defined by :

$$
R_{i, 2} \geq\left|z_{1}\right| \geq R_{i, 1}>0,\left|z_{1}\right| \geq R_{i, 3} \cdot\left|z_{2}^{m}\right|
$$

Here we have $R_{i, 2} \rightarrow \infty, R_{i, 1} \rightarrow 0$ and $R_{i, 3} \downarrow 1$ as $i \rightarrow \infty$.

Since $W=W_{1}^{\prime} \cup W_{2}$ and $W_{1}^{\prime}, W_{2}$ are quasi-Stein spaces, we can use Leray's theorem to calculate the $H^{i}\left(W, \mathcal{O}_{W}\right)$. We see that $H^{i}\left(W, \mathcal{O}_{W}\right), i \neq 1$ is as in lemma 3.3 , only $H^{1}\left(W, \mathcal{O}_{W}\right)$ is different. In fact we have :

$H^{1}\left(W, \mathcal{O}_{W}\right)=\left\{\sum_{n, m<0} a_{n, m} z_{1}^{n} z_{2}^{m} \mid\right.$ the powerseries converges on $\left.W_{1}^{\prime} \cap W_{2}\right\}$

So only the convergency condition of the powerseries has changed.

Now the action of $\gamma$ on $H^{1}\left(W, \mathcal{O}_{W}\right)$ is well-defined. We have :

$$
\begin{aligned}
\gamma\left(\frac{1}{z_{1}^{k} z_{2}^{l}}\right) & =\alpha^{-m k-l}\left(1+\lambda \frac{z_{2}^{m}}{z_{1}}\right)^{-k} z_{1}^{-k} z_{2}^{-l} \\
& =\alpha^{-m k-l} z_{1}^{-k} z_{2}^{-l} \sum_{i \geq 0}\left({ }_{i}^{-k}\right)\left(\lambda \frac{z_{2}^{m}}{z_{1}}\right)^{i}
\end{aligned}
$$

This powerseries is convergent on $W_{1}^{\prime} \cap W_{2}$ since $\left({ }_{i}^{-k}\right) \in \mathbb{Z}$ and therefore $\left|\left(i^{-k}\right)\right| \leq 1$. In fact we may forget about $z_{i}$-powers $\geq 0$, since in $H^{1}\left(W, \mathcal{O}_{W}\right)$ we are looking at powerseries $\sum_{k, l<0} a_{k, l} z_{1}^{k} z_{2}^{l}$ modulo monomials having a $z_{i}$-power $\geq 0$. So the series $\gamma\left(\frac{1}{z_{1}^{k} z_{2}^{l}}\right)$ stops as soon as $m i-l \geq 0$.

Lemma 3.7. Let $\gamma$ be a contraction of the form

$$
\gamma\left(z_{1}, z_{2}\right)=\left(\alpha^{m}\left(z_{1}+\lambda z_{2}^{m}\right), \alpha z_{2}\right), 0<|\alpha|<1,0<|\lambda| \leq 1
$$


Let $\mathfrak{L}_{\beta}$ be a line bundle on the Hopf surface $W / \Gamma$, where $\Gamma=<\gamma>$. Now we have :

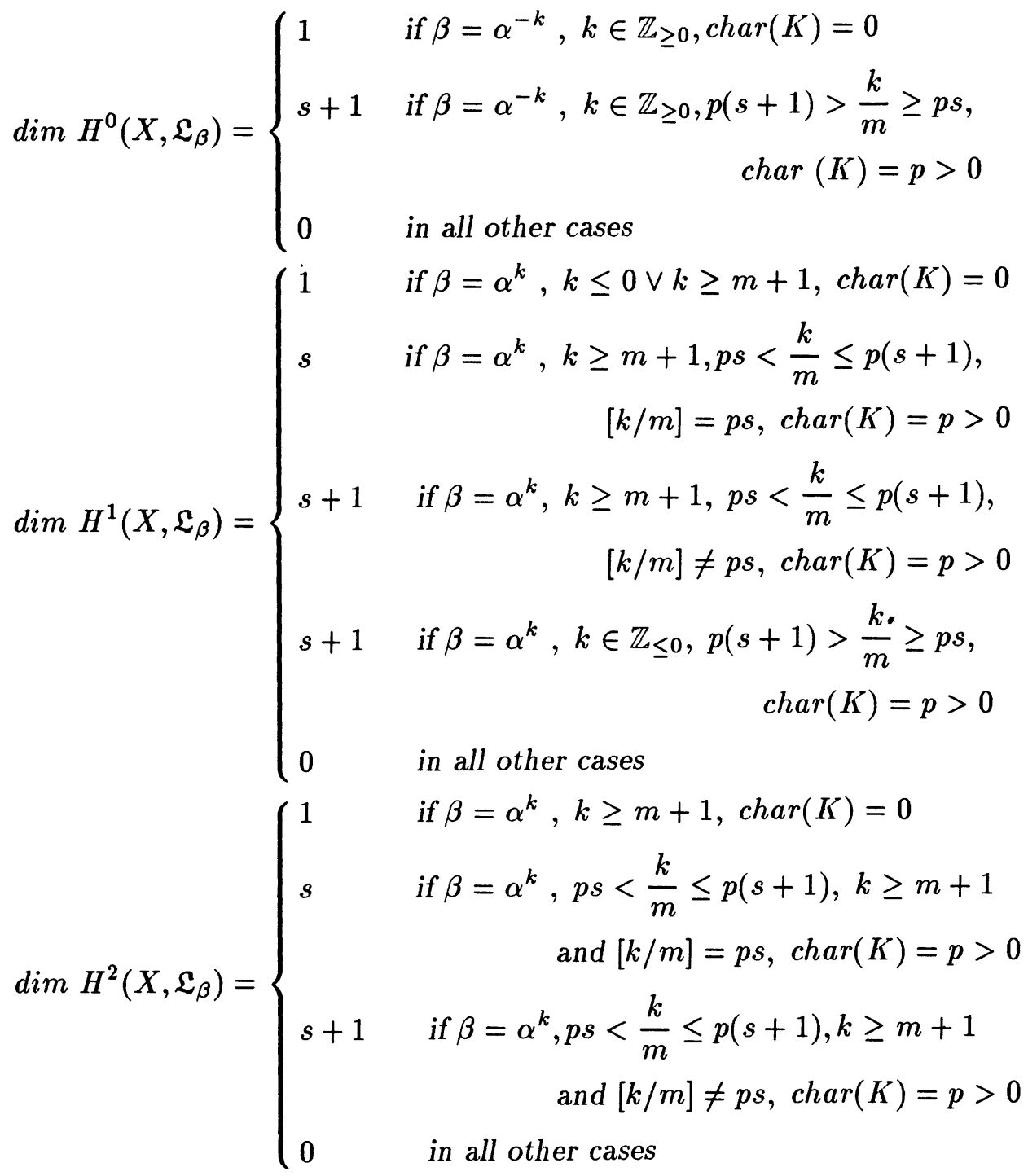

Proof. We may replace the coordinate $z_{1}$ by $\lambda^{-1} z_{1}$, so we can assume that $\lambda=1$ and that $\gamma$ has the form : $\gamma\left(z_{1}, z_{2}\right)=\left(\alpha^{m}\left(z_{1}+z_{2}^{m}\right), \alpha z_{2}\right)$.

We also replace the monomials $z_{1}^{k} z_{2}^{l}$ by $x^{k} z_{2}^{l+m k}$ where $x=\frac{z_{1}}{z_{2}^{m}}$, so $\gamma(x)=x+1$. 
So now we have :

$$
\begin{aligned}
& H^{0}\left(W, \mathcal{O}_{W}\right)=\left\{\sum_{k \geq 0, l-k m \geq 0} a_{k, l} x^{k} z_{2}^{l} \mid \text { the powerseries converges on } W\right\} \\
& H^{1}\left(W, \mathcal{O}_{W}\right)=\left\{\begin{array}{c}
\sum_{k<0, l-k m<0} a_{k, l} x^{k} z_{2}^{l} \mid \\
W_{1}^{\prime} \cap W_{2}
\end{array}\right\} \\
& H^{i}\left(W, \mathcal{O}_{W}\right)=0, i \geq 2 .
\end{aligned}
$$

We will first calculate $H^{0}\left(X, \mathfrak{L}_{\beta}\right)$. We have :

$$
\gamma\left(x^{k} z_{2}^{l}\right)=(x+1)^{k} \alpha^{l} z_{2}^{l}=\alpha^{l} \sum_{i=0}^{k}\left(\begin{array}{l}
k \\
i
\end{array}\right) x^{i} z_{2}^{l}, k \geq 0, l-k m \geq 0 .
$$

Now take $f \cdot e \in \mathfrak{L}_{\beta}$ such that $\gamma(f \cdot e)=f \cdot e=\gamma(f) \cdot \beta \cdot e$.

So we must have : $f=\beta \cdot \gamma(f)$. We can write :

$$
f=\sum f_{i}(x) z_{2}^{i} \Rightarrow \gamma(f)=\sum \alpha^{i} \gamma\left(f_{i}(x)\right) \cdot z_{2}^{i}, \gamma\left(f_{i}(x)\right)=f_{i}(x+1) .
$$

So we have : $\gamma(f)=\beta^{-1} \cdot f \Rightarrow f=f_{i}(x) z_{2}^{i}, \quad \beta=\alpha^{-i}, i-m \cdot \operatorname{deg}\left(f_{i}(x)\right) \geq 0$ and $\gamma\left(f_{i}(x)\right)=f_{i}(x)$.

Let $f_{i}(x)$ be given by $f_{i}(x)=\sum_{j=0}^{s} a_{j} x^{j}$. This gives us :

$$
\begin{aligned}
\gamma\left(f_{i}\right)=f_{i} & \Leftrightarrow \sum_{j=0}^{s} a_{j} x^{j}=\sum_{j=0}^{s} a_{j}(x+1)^{j}, s=\operatorname{deg}\left(f_{i}\right) \\
& \Rightarrow s \cdot a_{s}+a_{s-1}=a_{s-1} \\
& \Rightarrow s=0 \vee a_{s}=0 .
\end{aligned}
$$

Since $a_{s} \neq 0$, we have $s=0$. When $\operatorname{char}(K)=0$ we find $s=0$ and $f_{i} \in K$.

Therefore we have : $f=f_{i} z_{2}^{i}=c z_{2}^{i}, c \in K, \gamma(f . e) \stackrel{-}{=} \alpha^{i} \cdot \beta \cdot f e$

$$
\Rightarrow \begin{cases}\operatorname{dim} H^{0}\left(X, \mathfrak{L}_{\beta}\right)=1 & \text { if } \beta=\alpha^{-i}, i \geq 0 \\ \operatorname{dim} H^{0}\left(X, \mathfrak{L}_{\beta}\right)=0 & \text { otherwise. }\end{cases}
$$

If $\operatorname{char}(K)=p>0$ then we find $p \mid s$. It is easy to see that the polynomials $\left(x^{p}-x\right)^{j}$ are $\gamma$-invariant. Now we have : 


$$
\begin{aligned}
& f_{i}=\sum_{j=0}^{s} a_{j}\left(x^{p}-x\right)^{j}, \operatorname{deg}\left(f_{i}\right)=p s . \\
& f=f_{i} z_{2}^{i}, i-m \cdot \operatorname{deg}\left(f_{i}\right) \geq 0 \text { so } i-m p s \geq 0, \beta=\alpha^{-i} .
\end{aligned}
$$

This gives us :

$$
\left\{\begin{array}{l}
\operatorname{dim} H^{0}\left(X, \mathfrak{L}_{\beta}\right)=s+1 \text { if } \beta=\alpha^{-i}, i \geq 0 \text { and } p(s+1)>\frac{i}{m} \geq p s \\
\operatorname{dim} H^{0}\left(X, \mathfrak{L}_{\beta}\right)=0 \text { otherwise. }
\end{array}\right.
$$

Since there is for every $l \geq 0$ only a finite number of polynomials $x^{k} z_{2}^{l}$ with $k \geq 0$ and $l-k m \geq 0$ and $\gamma\left(x^{k} z_{2}^{l}\right)=\alpha^{l}(x+1)^{k} z_{2}^{l}$ and since there exist for at most one value of $l$ invariant polynomials $f_{i}(x) z_{2}^{l}$ such that $\gamma\left(f_{i}(x) z_{2}^{l} e\right)=f_{i}(x) z_{2}^{l} e$, we have again :

$$
\operatorname{dim} H^{0}\left(\Gamma, H^{0}\left(W, u^{*} \mathfrak{L}_{\beta}\right)\right)=\operatorname{dim} H^{1}\left(\Gamma, H^{0}\left(W, u^{*} \mathfrak{L}_{\beta}\right)\right) .
$$

We will now study the action of $\gamma$ on $\left.H^{1}\left(W, u^{*} \mathfrak{L}_{\beta}\right)\right)$. We have :

$$
\gamma\left(x^{-k} z_{2}^{-l}\right)=\alpha^{-l}(x+1)^{-k} z_{2}^{-l}=\alpha^{-l} \sum_{i=0}^{\infty}\left({ }_{i}^{-k}\right) x^{-k-i} z_{2}^{-l}, k>0, l>k m .
$$

We can forget the terms $x^{-k-i} z_{2}^{l}$ with $-l+(k+i) m \geq 0$, so we only have to look at a finite sum. Now take an element $f \cdot e \in \mathfrak{L}_{\beta \mid W_{1}^{\prime} \cap W_{2}}$ such that $\gamma(f e)=\beta \gamma(f) \cdot e=f \cdot e$. So we have $: f=\beta \cdot \gamma(f)$.

We can write $f=\sum f_{i}\left(x^{-1}\right) z_{2}^{i}$ where $f_{i}$ is a polynomial such that $i-\operatorname{deg}\left(f_{i}\right) \cdot m<0$. Therefore

$$
\gamma(f)=\sum \alpha^{i} \gamma\left(f_{i}\left(x^{-1}\right) z_{2}^{i}=\sum \alpha^{i} \gamma\left(f_{i}\left((x+1)^{-1}\right) z_{2}^{i} .\right.\right.
$$

So we have : $\gamma(f)=\beta^{-1} f \Rightarrow f=f_{i}\left(x^{-1}\right) z_{2}^{i}, \quad \beta=\alpha^{-i}, i-\operatorname{deg}\left(f_{i}\right) \cdot m<0$ and $\gamma\left(f_{i}\left(x^{-1}\right)\right) z_{2}^{i}=f_{i}\left(x^{-1}\right) z_{2}^{i}$ modulo monomials $x^{k} z_{2}^{i}$ with $i-k m \geq 0$.

Let $f$ be

$$
\begin{aligned}
f=f_{i}\left(x^{-1}\right) z_{2}^{i} & =\sum_{\substack{j>0 \\
i+j m<0}} a_{j} x^{-j} z_{2}^{i} \\
& =a_{s} x^{-s} z_{2}^{i}+\sum_{\substack{j>s \\
i+j m<0}} a_{j} x^{-j} z_{2}^{i}, a_{s} \neq 0 .
\end{aligned}
$$


Now $\gamma(f)=\beta^{-1} f$ implies that :

$$
\begin{aligned}
& \left(\overline{1}^{-s}\right) a_{s}+a_{s+1}=a_{s+1}, \beta=\alpha^{-i} \\
\Leftrightarrow & \left(\overline{1}^{-s}\right) a_{s}=0 \vee m s+m+i \geq 0 \\
\Leftrightarrow & \left(\overline{1}^{-s}\right)=-s=0 \vee m s+m+i \geq 0 .
\end{aligned}
$$

If $\operatorname{char}(K)=0$ then $s=0$ cannot occur since in $x^{-s} z_{2}^{i}$ we have $s>0$. So we must have $m s+m+i \geq 0$ and $s m+i<0$, since otherwise $f \equiv 0$.

This gives us :

$$
\begin{cases}\operatorname{dim} H^{0}\left(\Gamma, H^{1}\left(W, u^{*} \mathfrak{L}_{\beta}\right)\right)=1 & \text { if } \beta=\alpha^{-i}, i<-m \\ \operatorname{dim} H^{0}\left(\Gamma, H^{1}\left(W, u^{*} \mathfrak{L}_{\beta}\right)\right)=0 & \text { otherwise. }\end{cases}
$$

If $\operatorname{char}(K)=p>0$ then we have $p \mid s$ or $m s+m+i \geq 0, s m+i<0$.

It is easy to see that the powerseries $\left(\frac{1}{x^{p}-x}\right)^{r}$ is $\gamma$-invariant :

$$
\frac{1}{\left(x^{p}-x\right)^{r}}=\frac{1}{x^{p r}} \cdot \frac{1}{\left(1-x^{1-p}\right)^{r}}=x^{-p r}\left(\sum_{j=0}^{\infty} x^{(1-p) j}\right)^{r} \text {. }
$$

So we can use the polynomials :

$$
z_{2}^{i} . x^{-p r}\left(\sum_{j=0}^{j_{0}} x^{(1-p) j}\right)^{r}, i+m p r<0, r>0 .
$$

Here $j_{0}$ is taken such that $i+m\left(p r+j_{0}(p-1)\right) \geq 0$.

Furthermore we find an extra $\gamma$-invariant monomial $x^{-r} z_{2}^{i}$ if $-(r+1) \leq \frac{i}{m}<-r<0$ and $r \neq p \cdot s$ for some $s \in \mathbb{Z}_{>0}$. So we now have :

$$
\left\{\begin{aligned}
\operatorname{dim} H^{0}\left(\Gamma, H^{1}\left(W, u^{*} \mathfrak{L}_{\beta}\right)\right) & =s \text { if }-p(s+1) \leq \frac{i}{m}<-p s, \beta=\alpha^{-i}, \\
-i & >m \text { and }-(r+1) \leq \frac{i}{m}<r \Rightarrow r=p s, \\
\operatorname{dim} H^{0}\left(\Gamma, H^{1}\left(W, u^{*} \mathfrak{L}_{\beta}\right)\right) & =s+1 \text { if }-p(s+1) \leq \frac{i}{m}<-p s, \beta=\alpha^{-i}, \\
-i>m \text { and }-(r+1) \leq \frac{i}{m}<r \Rightarrow r \neq p s & \text { otherwise. }
\end{aligned}\right.
$$


Again we have $: \operatorname{dim} H^{1}\left(\Gamma, H^{1}\left(W, u^{*} \mathfrak{L}_{\beta}\right)\right)=\operatorname{dim} H^{0}\left(\Gamma, H^{1}\left(W, u^{*} \mathfrak{L}_{\beta}\right)\right)$.

So we have : $\operatorname{dim} H^{2}\left(X, \mathfrak{L}_{\beta}\right)=\operatorname{dim} H^{0}\left(\Gamma, H^{1}\left(W, u^{*} \mathfrak{L}_{\beta}\right)\right)$.

Furthermore we have : $\operatorname{dim} H^{1}\left(X, \mathfrak{L}_{\beta}\right)=\operatorname{dim} H^{0}\left(X, \mathfrak{L}_{\beta}\right)+\operatorname{dim} H^{2}\left(X, \mathfrak{L}_{\beta}\right)$.

Theorem 3.1. Let $\Gamma=\langle\gamma\rangle$ be generated by a contraction $\gamma$.

Let $\mathfrak{L}_{\beta}$ be a line bundle on the Hopf surface $X=W / \Gamma$. We now have :

a) $\chi\left(\mathfrak{L}_{\beta}\right)=0$

b) There exists an unique line bundle $\mathfrak{L}$ such that :

$$
\forall \mathfrak{L}_{\beta} \quad H^{2-i}\left(X, \mathfrak{L} \otimes \mathfrak{L}_{\beta}^{-1}\right)=H^{i}\left(X, \mathfrak{L}_{\beta}\right) .
$$

c) We have $\mathfrak{L}^{\cdot}=\mathfrak{L}_{\alpha_{1}, \alpha_{2}}=\mathfrak{L}(K)$.

Proof. The Euler characteristic $\chi\left(\mathfrak{L}_{\beta}\right)$ is defined by :

$$
\chi\left(\mathfrak{L}_{\beta}\right)=\sum_{i=0}^{2}(-1)^{i} \operatorname{dim} H^{i}\left(X, \mathfrak{L}_{\beta}\right) .
$$

In the lemmas $3.4,3.5$ and 3.7 we have used the following fact :

$$
\operatorname{dim} H^{1}\left(X, \mathfrak{L}_{\beta}\right)=\operatorname{dim} H^{0}\left(X, \mathfrak{L}_{\beta}\right)+\operatorname{dim} H^{2}\left(X, \mathfrak{L}_{\beta}\right) .
$$

This proves statement $a$ of the theorem.

The Serre duality in part $b$ can be found by direct verification. We shall only determine the only possible line bundle $\mathfrak{L}$. We have :

$$
\left\{\begin{array}{l}
\operatorname{dim} H^{0}(X, \mathfrak{L})=\operatorname{dim} H^{2}\left(X, \mathfrak{L} \otimes \mathfrak{L}^{-1}\right)=\operatorname{dim} H^{2}\left(X, \mathfrak{L}_{1}\right)=0 \\
\operatorname{dim} H^{2}(X, \mathfrak{L})=\operatorname{dim} H^{0}\left(X, \mathfrak{L} \otimes \mathfrak{L}^{-1}\right)=\operatorname{dim} H^{0}\left(X, \mathfrak{L}_{1}\right)=1
\end{array}\right.
$$

Since $\operatorname{dim} H^{2}\left(X, \mathfrak{L}_{1}\right)=1$, we have $\mathfrak{L}=\mathfrak{L}_{a}$ with $a=\alpha_{1}^{k} \alpha_{2}^{l}, k, l \in \mathbb{Z}_{>0}$.

Now take $\beta=\alpha_{1}^{r} \alpha_{2}^{s}, r, s>0$, then we have :

$0 \neq \operatorname{dim} H^{2}\left(X, \mathfrak{L}_{\beta}\right)=\operatorname{dim} H^{0}\left(X, \mathfrak{L}_{\alpha_{1}^{r-k} \alpha_{2}^{s-l}}\right) \Rightarrow-r+k \geq 0,-s+l \geq 0$.

So only $k=l=1$ can satisfy $b$, therefore $\mathfrak{L}=\mathfrak{L}_{\alpha_{1} \alpha_{2}}$.

The canonical divisor $K$ on $X$ is given by

$$
K= \begin{cases}z_{1}^{-1} z_{2}^{-1} \cdot d z_{1} \wedge d z_{2} & \text { if } \gamma\left(z_{1}, z_{2}\right)=\left(\alpha_{1} z_{1}, \alpha_{2} z_{2}\right) \\ z_{2}^{-(m+1)} \cdot d z_{1} \wedge d z_{2}, & \text { if } \left.\quad \gamma\left(z_{1}, z_{2}\right)=\left(\alpha^{m}\left(z_{1}+z_{2}^{m}\right), \alpha z_{2}\right)\right)\end{cases}
$$


Clearly we have : $\mathfrak{L}(K)=\mathfrak{L}_{\alpha_{1} \alpha_{2}}$. This proves part $c$ of the theorem.

Remark : Theorem 3.1 gives us a Riemann-Roch theorem on the Hopf surface $X$.

Indeed we have : $l(D)-s(D)+l(K-D)=\frac{1}{2} D(D-K)+1+p_{a}$

$$
\Rightarrow \chi(\mathfrak{L}(D))=\frac{1}{2} D(D-K)+1+p_{a}
$$

Taking $D=K$ we have $0=1+p_{a} \Rightarrow p_{a}=-1$.

Taking $D=n \cdot K$ we have $\frac{1}{2}\left(n K^{2}-n^{2} K^{2}\right)=0 \Rightarrow K^{2}=0$.

So we have :

$$
D^{2}=D \cdot K \text { for every } D
$$

\section{REFERENCES}

[BGR] S. BOSCH, U. GÜNTZER, R. REMMERT, Non-archimedean analysis, Springer Verlag, 1984.

[CE] H. CARTAN, S. EILENBERG, Homological Algebra, Princeton Univer. Press, 1956.

[FP] J. FRESNEL, M. van der PUT, Géométrie analytique rigide et applications, Progress in Math. 18, Birkhäuser, (1981).

[GG] L. GERRITZEN, H. GRAUERT, Die Azyklizität der affinoiden Überdeckungen, in "Global Analysis, Papers in honor of K. Kodaira", Univ. Tokyo Press and Princeton Univ. Press, 1969, 159-184.

[GP] L. GERRITZEN, M. van der PUT, Schottky groups and Mumford curves, Lectures Notes in Math. 817, Springer Verlag, 1980.

[G] L. GRUSON, Théorie de Fredholm p-adique, Bull. Soc. Math. France 94 (1966), 67-95.

[H] R. HARTSHORNE, Algebraic Geometry, Springer Verlag, 1977.

[Ki.1] R. KIEHL, Der Endlichkeitssatz für eigentliche Abbildungen in der nicht-archimedischen Funktionentheorie, Invent. Math. 2 (1967), 191-214.

[Ki.2] R. KIEHL, Theorem $A$ und theorem $B$ in der nicht-archimedischen Funktionen-theorie, Invent Math 2 (1967), 256-273.

[KKMS] G. KEMPF, F. KNUDSEN, D. MUMFORD, B. SAINT-DONAT, Toroidal Embeddings, Lecture Notes in Math. 339, Springer Verlag, 1973.

[Ko.1] K. KODAIRA, On the structure of compact complex analytic surfaces II, Amer. J. Math. 88 (1966), 682-721. 
[Ko.2] K. KODAIRA, On the structure of compact complex analytic surfaces III, Amer. J. Math. 90 (1969), 55-83.

[Mus.1] G.A. MUSTAFIN, p-adic Hopf varieties, Functional Anal. and its Appl. 11 (1977), 234-235.

[Mus.2] G.A. MUSTAFIN, Nonarchimedean Uniformization, Math. USSR Sbornik 34 (1978), 187-214.

[O.1] T. ODA, Lectures on Torus Embeddings and Applications, Tata Inst. of Fund. Research 58, Springer Verlag, 1978.

[O.2] T. ODA, Convex Bodies and Algebraic Geometry, Springer Verlag, 1988.

[vdP] M. van der PUT, $A$ note on p-adic uniformization, Proceedings of the K.N.A.W. A90 (1987), 313-318.

[U] K. UENO, Compact Rigid Analytic Spaces - with special regard to surfaces -, in "Algebraic Geometry, Sendai, 1985", Advanced Studies in Pure Math. 10 (1987), North-Holland, 765-794.

Max Planck Institut für Mathematik

Gottfried-Claren-Strasse 26

5300 Bonn 3

Germany . 